SF 751

.K64

Copy 1

Domestic Animals

and Poultry

Their Cause,

Symptoms and

Treatment

Price, \$2.00 


$$
\approx
$$






\title{
Diseases of Domestic Animals and Poultry
}

\author{
Their Cause, Symptoms \\ and Treatment
}

BY

$$
\text { C. J. Korinek, V. S. }
$$

AND

A. W. Korinek, V. S., B. V. Sc.

\section{Published by}

KORINEK REMEDY COMPANY

Kenton Station

PORTLAND - OREGON 


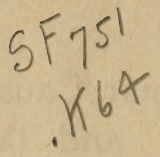

JEROME ADVERTISING CO.

Broadway Bldg.

Portland, Ore.

BUSHONG \& CO.

Printers

Portland, Oregon

Illustrations in this book are from photographs furnished by the Rural Spirit. 


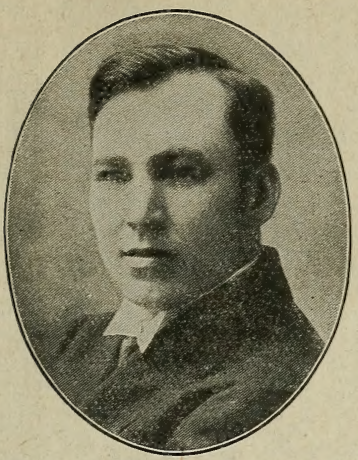

C. J. KORINEK, V. S.

Graduate of Ontario Veterinary College, in affliation with the University of Toronto; Oregon State Veterinarian under two Governors. Served four years as President of the Oregon State Veterinary Medical Board of Examiners.

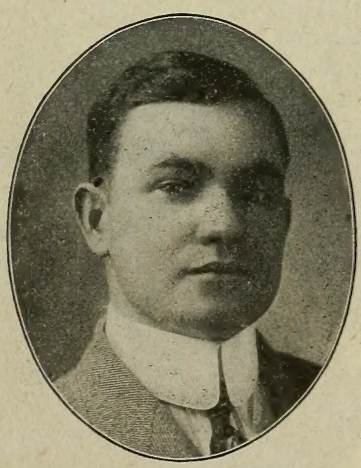

A. W. KORINEK, V. S., B. V. S. C.

Graduate of Ontario Veterinary College, under the control of the Department of Agriculture of Ontario; Graduate Veterinary Department University of Toronto. Served as Deputy State Veterinarian of Massachusetts. Pure Food, Meat and Milk Inspector for Williamstown, Mass. 


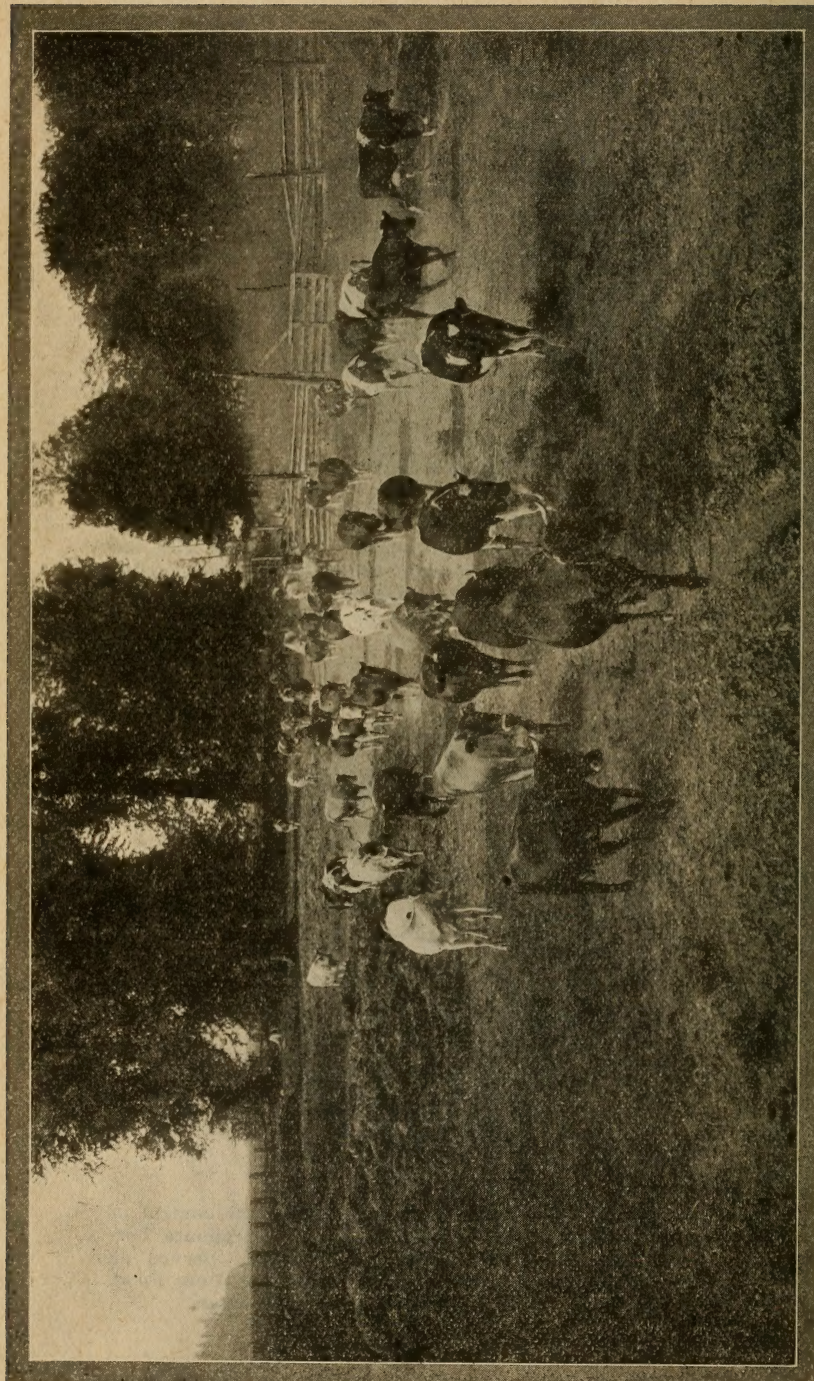

台 


\section{Preface.}

Realizing the need for a condensed, authoritative work on the prevention, cause, symptoms and treatment of the most prevalent Diseases of Domestic Animals and Poultry, the authors have directed their best energies towards producing such a treatise.

There is always present the difficulty of presenting a technical suhject in untechnical phraseology, so we have adhered to plain, everyday language, avoiding technical terms as far as possibleand frequently at the cost of grammatical accuracy.

We have tried to be as brief as is consistent with clearness, for a condensed work of this sort does not permit of going into detail nor writing many unnecessary paragraphs.

All of the preparations and prescriptions, as well as instructions for prevention, cause, symptoms and treatment are original, and the result of our long experience in the practice of Veterinary Medicine, Surgery, Research and Experimental Work pertaining to Domestic Animals and Poultry.

The authors feel that such a work will be of untold help to busy Stock and Poultry raisers for quick reference, and if such is the case, our efforts will not have been in vain.

In conclusion we wish to say that we desire the co-operation of all Stock and Poultry raisers, and will highly appreciate ideas or suggestions that will tend to improve later editions of this little book.

THE AUTHORS. 


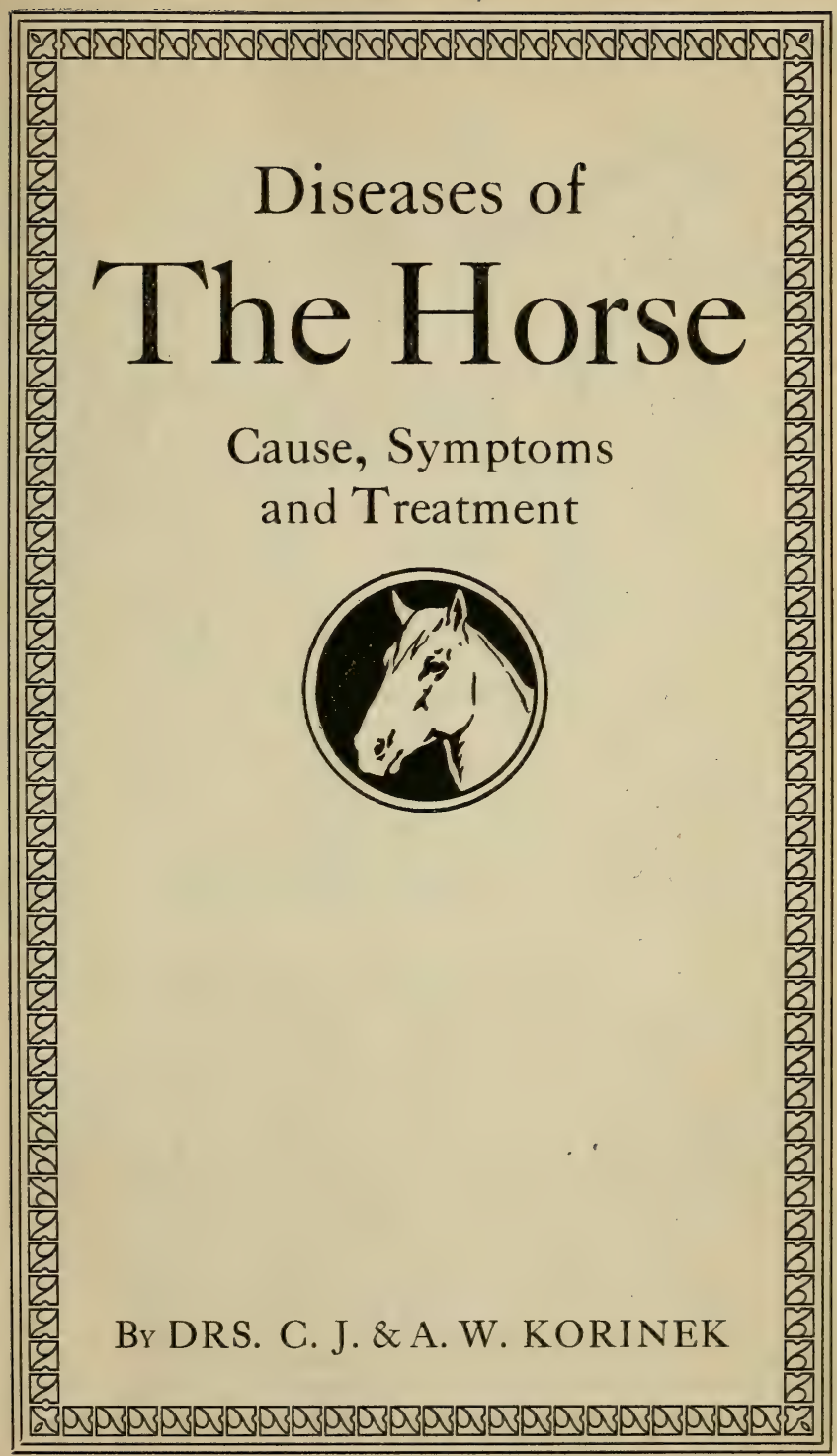




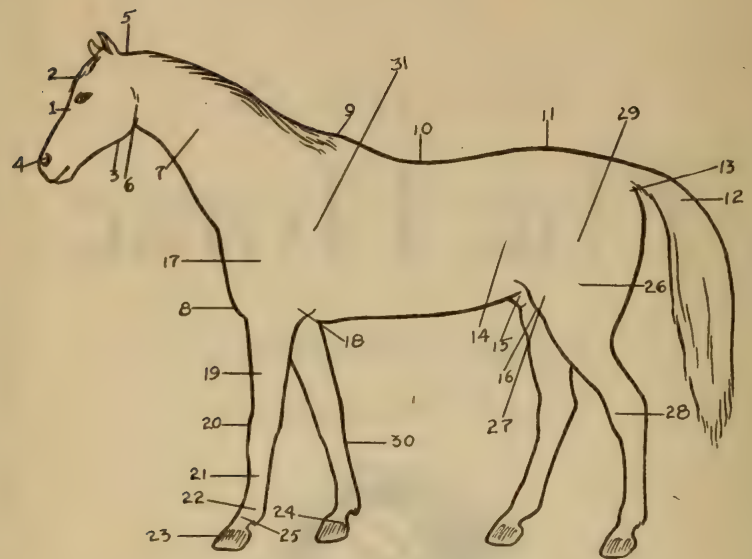

POINTS OF THE HORSE

1 Face

2 Forehead

3 Lower Jaw

4 Nostrils

5 Poll

6 Throat

7 Neck

8 Breast

9 Withers

10 Back

11 Croup

12 Tail

13 Anus

14 Flank

15 Sheath

16 'i esticles

17 Point of Shoulder

18 Elbow

19 Forearm

20 linee

21 Canon

22 Fetlock Joint

23 Hoof

24 Coronet

25 Pastern
26 Thigh

27 Stifle

28 Hock

29 Hip

30 Splint of Bone

31 Angle of Shoulder

SEAT OF DIFFERENT DISEASES OF THE HORSE

5 Poll Evil

9 Fistulous Withers

17 Shoulder Joint Lameness

18 Capped Elbow-Shoe Boil

23 Laminitis or Founder-Navicular Disease

20 Broken Knee

27 Stifle Joint Lameness

28 Spavin - Thorough Pin Curb-Capped Hock

29 Hip Joint Lameness

30 splint

31 Sweeney

25 Ringbone

24 Sidebones 


\section{Abscess.}

CALSE. Bruises and injurics. They are also seen in complication with various other diseases, perhaps the most common being Distemper, Laryngitis, Pharyngitis, Blood Poisoning, Etc.

SYMPTOMS. Symptoms will vary, according to the development of the injuries or diseases. It may not be noticed at first, but upon careful examination small tortuous lines will be observed running from the point of irritation. In many cases a swelling is noticed which is hot, painful and throbbing and enlarges rapidly. In two or three days the soreness and heat gradually subside, but the abscess continues to grow. The hair falls from the affected parts and in a short time the abscess discharges, and the cavity gradually fills up and heals by granulation.

TREATMENT. In all cases hasten the ripening process as much as possible with hor water and poultices. Dr. Korinek's Absorbent Blister rubbed well over the surface is advisable where the abscess is slow in developing. It is common with veterinarians to lance an abscess as soon as possible, but this requires skill and practice. I cannot recommend stock-owners to perform this operation, as it requires an exact knowledge of anatomy. It will usually be found a safer and better plan to encourage the full ripening of an abscess and allow it to open itself, as it will heal better and quicker, and you take no chances of infection as with an instrument. When opened do not squeeze the abscess to any extent, but press gently with a clean hand or cloth at first to remove the clot, and after this simply keep open by washing with Dr. Korinek's Dip and Disinfectant, one teaspoonful to a pint of water; carbolic acid 3 per cent, or bichloride of Mercury, one part to one thousand parts water.

\section{Abortion In Mares.}

CALSE. Quality and quantity of food, poorly lighted, ventilated or drained stables, mare falling, or slipping, sprains, kicks, hard fast work, or eating poisonous vegetation.

SYMPTOMS. Mare will show signs of colic, the outer portion of the womb will be swollen, and if the colicky symptoms continue there will be a watery discharge and the membranes covering the foal will become noticeable. The animal strains when trying to lie down or get up.

TREATMENT. Place animal in comfortable quarters and blanket if cnilly. When colicky signs are present give one of Dr. Korinek's Colic Capsules as directed on package. To stop the straining give Tr. Opii, one ounce placed in a capsule and which should be given with a capsule gun; one or two doses are usually sufficient, as the mare will either abort or the dangerous period has passed. Keep animal quiet, feed good nutritious food and give pure water with the chill taken off, in small quantities, but often. Disinfect the 
mare's quarters. A good general tonic is indicated-one that is strengthening and assists nature in throwing off impurities from the blood. Give Dr. Korinek's Tonic Capsules as directed on package.

\section{Anaemia.}

CAUSE. Poor quality and insufficient quantity of food, bad surroundings, overwork, lack of exercise, drain on the system from acute or chronic disease. Can also be brought about by excessive heat, cold, or pressure, and by diminution of the caliber of the arteries. Poisons in the blood, suppurations, wounds, repeated purging or bleeding.

SYMPTOMS. The visible mucous membranes of the nose, eyes and mouth are pale and sometimes have a yellow appearance. There is weakness, temperature of the body is lower than normal; pulse weak, legs cold to the feet, cold sweats are often present, breathing is quickened, especially in the last stages, animals tire easily, appetite and digestion becomes poor, swelling of the legs, and the under surface of the abdomen, sheath and udder, the skin becomes rough and dry.

TREATMENT. Remove the cause-if it can be found. Give one of Dr. Korinek's Physic Capsules according to indications, that is to say, if the animal is constipated, one Physic Capsule, then give one of Dr. Korinek's Tonic Capsules three times a day. Try to build up the condition of the animal by giving the proper quantity and quality of food. Give pure water to drink, also provide plenty of fresh air and sun light. Turn out to grass when the weather is favorable. This treatment should be continued until the animal shows signs of improvement, then the Tonic Capsules can be discontinued and replaced by Dr. Korinek's Stock Tonic.

\section{Apthae.}

\section{(Sore Mouth and Tongue-Pustulai Stomatitis.)}

CAUSE. Superficial eruptions of the mucous membranes of the mouth and tongue. Frequently seen during convalescence of intermittent fever. This condition may also follow diseases of the digestive system, as Indigestion, etc., due to the blood absorbing toxic materials which break out in the form of pustules about the mouth and the whole alementary canal (stomach and intestines).

SYMPTOMS. The appetite is impaired, the mouth hot, the pulse not much affected as a rule, the temperature is slightly elevated, the animal is unable to masticate and small vesicles appear and eventually terminate into pustules which burst and discharge a small amount of pus at the parts where the sores are the deepest.

TREATMENT. Remove the cause if possible. Feed clean, soft food that is easily digested, as hot wheat bran mashes and steamed 
rolled oats, vegetables, etc. For a mouth wash dissolve the following: One dram of Copper Sulphate, one dram of Chlorate of Potash, one dram of Boracic Acid in clean, hot water and syringe out the mouth two or three times a day. To their drinking water add one ounce of Hyposulphite of Soda twice a day. Where the appetite is impaired administer Dr. Korinek's Tonic Capsules as directed on package.

\section{Azoturia.}

CAUSE. This disease is always due to work after a period of idleness, during which the animal has been liberally fed. It is found principally among highly-fed draft horses, and never in animals which are regularly worked. Light breeds of horses are also susceptible to this disease.

SYMPTOMS. Attack is sudden and usually appears when the horse has traveled a short distance after having been stabled for a few days. The characteristic symptoms of this disease in an animal are: Excitability without apparent cause; actions seem to indicate injury of the hind quarters or loins. Animal has a peculiar gooserumped look owing to the muscles over the quarters being violently contracted and are hard on pressure. One hind limb is generally advanced in front of the other, and on attempting to put weight on it the hind quarters will drop until at times the hocks almost touch the ground. Sometimes a front leg is affected. The breathing is hurried. Animal is bathed in sweat and is in such agony that it will seize almost anything with its teeth. Although the pulse is hard and frequent, the internal temperature, even in severe cases, seldom rises to any marked extent. The urine is dark-red to dirty-brown color. Owing to the stoppage of the worm-like movement of the bowels, there is generally constipation and retention of the urine. Sometimes the symptoms are milder than here described. In other cases the animal falls to the ground and continues to struggle in a delirious, half-paralyzed state until he dies. Sometimes this disease is mistaken for colic or acute indigestion, but it can readily be distinguished by the color of the urine.

TREEATMENT. At the first symptoms, stop and blanket the animal and let stand from one to three hours; then move to the nearest shelter, keeping as quiet and comfortable as possible, as excitement aggravates the disease. Give one of Dr. Korinek's Physic Capsules followed by one of Dr. Korinek's Kidney Capsules every six hours. Injections of soap and warm water are beneficial. Immerse a blanket in hot water and place over the loins, covering with a dry blanket; or, if this is impracticable, take a liniment-such as Dr. Korinek's-and rub in like a shampoo over the loins. It may be necessary to draw off the urine which is sometimes retained, and it is best to secure the services of a skillful veterinarian. Allow the animal to drink often, though in small quantity, of pure water with the 
chill taken off. If he is unable to stand on his feet, it is well to turn him from side to side every six hours. It is also advisable to fill bags with hay and place against his shoulders to prevent him lying flat on his side, as this may cause congestion of the lungs. Avoid drenching - it is dangerous. Should signs of colic appear and the animal bloat, give one of Dr. Korinek's Colic Capsules every hour until the colicky signs disappear.

\section{Bleeding After Castration}

If bleeding is from the little artery in the back portion of cord, it will generally stop of its own accord, but if it should continue to bleed for thirty minutes throw clean, cold water against the part.

When bleeding is from the large artery in front of the cord, it is considered dangerous. The artery should be tied with a silk thread if possible, or twisted with a pair of forceps. Occasionally the artery cannot be found, in which case the hole in the scrotum should be plugged with a clean cloth saturated with tincture of iron, which will clot the blood and thus close the artery.

\section{Blood Poisoning or Septicaemea or Pyaemia}

CAUSE: By the popular term "Blood-Poison" is meant a state of constitutional disturbance brought on by the entrance of putrid products-usually from a wound-into the blood. As a rule some pressure or inoculation is necessary for the introduction of poison into the circulation; hence, the necessity of free drainage and thorough disinfection of the wound, and the only hopeful cases are those in which by this means the supply of poison may be cut short.

SYMPTOMS. It is introduced through any wound or abrasion, whether due to injury, disease or by an operation. Signs of septic poison are heat, pain and swelling.

TREATMENT-Both internal and external treatment is necessary. See that the wound has good drainage and wash with some good antiseptic-Dr. Korinek's Dip or carbolic acid; follow with application of Dr. Korinek's Antiseptic Gall-powder. The above treatment should be repeated twice daily. Also give one of Dr. Korinek's Tonic Capsules night and morning; they contain intestinal antiseptics which are very valuable in the treatment of bloodpoisoning. Feed soft laxative feed and green grass if possible.

\section{Bots-Gastrophilis}

The presence of bots in a horse can, of course, be of no possible advantage to him, but their presence in small numbers as a rule produce little or no ill effects. 
If their number be large they become a source of debility and irritation. In practically ali cases they produce indigestion, especially among young horses; also loss of condition, colic and even death.

CAUSE. The bot flies which lay their eggs during the Autumn on the skin and hair of the animal. These eggs on becoming hatched (in from 20 to 25 days) produce small worms which irritate the skin by their movements and thus cause the horse to lick them off and take them into his mouth, with the result that they gain access to various parts of the intestinal canal. The bot having selected its place of residence attaches itself to the membranes lining the stomach and intestines and derives its sustenance during its stay from the wound made by its hooks. In the summer the larva, after living inside the horse for about ten months, quits its hold and is expelled with the feces. Having concealed itself near the surface of the ground it becomes changed into a chrysalis from which the gadfly issues after an inactive existence of from thirty to forty days. The female fly becomes impregnated, lays her eggs on those parts of the horse from which they can be most easily licked off and thus completes her cycle of existence.

SYMPTOMS. Membranes about the eyes and mouth are very pale as though the animal had lost a large quantity of blood; will also be subject to colicy attacks, hair faded, dull, rough appearance, appetite poor and develops a pot belly.

PREVENTION. The best means of prevention are spraying your horses with Dr. Korinek's fly dope; it prevents the gadfly from depositing her eggs on the animals.

TREATMENT: Withhold all food from twelve to twenty-four hours, then give one of Dr. Korinek's Worm Capsules; follow in four to six hours with one of Dr. Korinek's Physic Capsules. If the animal's hair is rough and condition poor, give Dr. Korinek's Tonic Capsules or Stock Tonic as directed on package.

\section{Bronchitis}

CAUSE. It may be the result of debility, constitutional diseases, inhalation of impure air, smoke, gases. Sometimes brought on by drenching by the escape of liquid into the wind pipe. Remember a horse cannot breathe through his mouth. It may also be caused by sudden chill, foreign bodies in wind pipe, micro organisms or it may be associated with influenza, glanders, lung fever, etc.

SYMPTOMS. Sore throat, loss of appetite, thirst, animal appears dull, membranes of the mouth, eyes and nose are reddened; urine is scanty and highly-colored, cough dry and husky. After two or three days the cough becomes looser and a frothy, sticky, mucus of a yellowish color is present, gradually becoming pus-like, after 
which the animal seems somewhat relieved. In the first stages the pulse is soft and weak but frequently the temperature is high, ranging from 105 to $106 \mathrm{~F}$; the breathing is quick and more or less difficult.

TREATMENT. Place the horse in a clean, comfortable, wellventilated stall, excluding drafts; blanket if the weather is chilly; also rub the legs and bandage them. Inhalation of steam from hot water and turpentine is beneficial. Give one teaspoonful of $\mathrm{Dr}$. Korinek's Distemper Remedy every four hours. If the animal shows signs of chilling, and has fever, give one of Dr. Korinek's Fever Capsules every six hours. In severe attacks it is well to apply Dr. Korinek's Absorbent Blister or Liniment to the throat and windpipe. The animal should be fed on soft food, such as hot bran mashes, grass, carrots, kale, apples, or steamed oats. Should a tonic seem necessary, give Dr. Korinek's Stock Tonic, as directed.

\section{Capped Knee}

CAUSE. Bruises from pawing or striking objects with the knee, falling on the ground, etc., are perhaps the most common causes.

SYMPTOMS. It may be a simple bruise or it may be a severe wound. There is always swelling, heat and pain present. The joint becomes stiff and interferes with the movement of the leg. Under careful treatment the swelling and enlargement disappear.

TREATMENT. Relieve the inflammation by fomenting with hot water if the skin is broken, then apply Dr. Korinek's Antiseptic Gall Powder. To reduce enlargement use Dr. Korinek's Absorbent Blister as directed.

\section{Capped Hock}

CAUSE. Some horses have the habit of rubbing or striking their hocks against the partition of their stalls. May also be produced by kicks from other horses, or hocks may be bruised by the singletree.

SYMPTOMS. An enlargement at the point of the hock, which may run up along the tendons and muscles of the leg. Repeated injuries cause the hock to enlarge and become flabby, and in some cases it contains a bloody serum or pus.

TREATMENT. Do not attempt to lance the puffy swelling or you may produce an open joint. The best plan is to remove the cause; then apply Dr. Korinek's Absorbent Blister as directed on package every forty-eight hours for one week.

If the enlargement has not disappeared in two or three weeks, repeat the above treatment. 


\section{Choking}

This term applies to obstruction of the gullet as well as that of the windpipe.

CAUSE. Too rapid eating, by which pieces of carrots or other roots or a quantity of dry food become lodged in the gullet. Although obstructions of the windpipe, caused while drenching, or food entering the lungs, will kill an animal in a very short time, obstructions in the gullet may not prove fatal for several days.

TREATMENT. No time should be lost in removing the cause, which may be done by gentle manipulation. If this does not prove successful, secure the services of a veterinarian. He will use a probang or inject sweet oil or olive oil into the gullet with a hypodermic syringe. In administering drenches with the object of dislodging the obstruction, remember that they are apt to "go the wrong way" and give rise to lung complications.

Stoppage of the windpipe from solid substance will usually cause death very shortly. If from liquid and it gains entrance to the lungs, it should be treated as lung fever.

\section{Coffin Joint Lameness-Navicular Disease}

CAUSE. Driving young animals on hard roads. Always found in the front feet, owing no doubt to the fact that the front feet support largely the weight of the body.

SYMPTOMS. The symptoms are very hard to detect. As a rule the animal will point the affected foot when at rest even before there is any lameness present. While at work he apparently goes sound, but when placed in the stable or when stopped on hard ground, one foot will be set out in front of the other and resting on the toe. It will be noticed that, the animal takes a few lame steps and then goes well again. Again he may be lame for a day, or he may leave the stable in the morning apparently well and sound and go lame during the day. In the course of time he will develop a severe case of lameness which may last for five or six days. These spells are intermittent and finally he becomes permanently lame, and the more he is driven the greater the lameness, and he steps short, wears the toe of the shoe, stumbles, falls on his knees when the road is rough. Sometimes both front feet are affected and the shoulders will be stiff. When put to work he sweats from pain; there will be extreme heat about the foot and he will flinch from pressure on the foot.

Comparatively few recoveries are made from this disease.

TREATMENT. First remove the shoe, if the foot is inflamed, poultice with bran or flaxseed. After the inflammation disappears clean the foot, clip the hair from around the top of the hoof and apply Dr. Korinek's Absorbent Blister, rubbing it in well, then turn the animal out to pasture. Repeat this treatment in a month. Animals affected with this disease should be put to slow and easy work. 


\section{Constipation}

CAUSE. Indigestible foods, irregular feeding, lack of or too much exercise, insufficient secretion of digestive materials, strictures, ruptures, paralysis, worms, folding and twisting of the intestines, which frequently occurs in old age.

SYMPTOMS. The animal cannot expel the contents of the intestines, which frequently causes colicky pains. Death from this form of constipation is generally due to rupture of the intestines, when due to indigestible foods or irregular feeding. Lack of or too much exercise seldom produces death, although the animal may not pass any fecal matter for a week.

TREATMENT: Give one of Dr. Korinek's Physic Capsules every eighteen hours until the animal has a passage, then give one of Dr. Korinek's Colic Capsules every six hours, as they will stimulate the worm-like action of the bowels and strengthen the heart action. Give the animal plenty of water, if water is cold, take of $\mathrm{f}$ the chill. If animal will eat, feed easily digested food, such as grass, carrots, turnips, apples, hot bran mash or steamed rolled oats. Give the animal injection of soap and warm water containing a few drops of turpentine. It is best to elevate the animal's hind parts when giving injection, as they can retain it much longer.

If due to worms give Dr. Korinek's Worm Capsules as directed on package.

\section{Colt Constipation}

CAUSE. Improper digestion of its mother's milk, especially when overheated or allowed to nurse too much or not enough.

SYMPTOMS. The colt appears stupid; does not care to move about but lies flat on either side and shows signs of great pain.

TREATMENT. Give two tablespoonfuls of Cascara Sagrada. Great care must be exercised in administering the medicine to place it well back on the tongue; do not hold the nose high or some of the liquid may enter the lungs; it is much better to waste some of the medicine. One of the most important factors in the treatment of Colt Constipation is rectal injections; they relieve temperature, gases and pain, promoting the worm-like action of the bowels and liquifying their contents.

\section{Colt Diarrhoea}

CAUSE: Specific infection, the action of which is favored by unsanitary conditions, irregular feeding or permitting the colt to nurse when the mother is overheated or out of condition.

SYMPTOMS: Frequent watery discharges, sometimes tinged with blood, and as the disease progresses the colt shows signs of great pain. If not treated promptly the disease will terminate fatally in the course of six or ten days. 
TREATMENT: Determine the exact cause, if possible, and remove it. If the colt has not been weaned, attention should at once be given the mare and if anything is wrong with her, it may be best to take the little patient away from its mother and feed it on cow's milk sweetened with sugar. Give two tablespoonfuls of castor oil on the tongue; this will remove the irritant within the bowels (Dr. Korinek's Calf Scour Remedy will complete the cure within a few days. Great care is necessary in giving medicines or the liquid may enter the lungs.) Do not pull the tongue or hold the head too high, and permit the animal to swallow slowly. Remember that sanitary surroundings are essential in the treatment of all diseases.

\section{Colic-Spasmodic}

Owing to the great length of their intestines, horses often suffer from colic, which is a common but severe abdominal disorder due to faulty digestion.

CAUSE: Generally caused by overwork, change of food, overfeeding, sudden chilling, etc. The violence of the attack in either spasmodic or flatulent forms often kills the horse.

SYMPTOMS: If animal is tied, it will show uneasiness, switch the tail, lie down, get up, etc. When loose, will walk nervously about, then try to lie down; in some cases will roll on back and attempt to remain in that position.

The temperature rises with the severity of the attack and breathing becomes labored and fast, the animal sweats in spots. There may be either diarrhoea or constipation present.

The following symptoms are always regarded as very unfavorable: Legs cold to the feet, tip of ears cold, twitching of muscles, cold sweats, when the mucuous membrane assumes a dark color due to congestion.

TREATMENT: In all ordinary cases of colic give Dr. Korinek's Colic Capsules every hour until relief is obtained. Also give one or two of Dr. Korinek's Physic Capsules, except when treating a mare that is heavy with foal, or an animal having diarrhoea.

Give an injection of soap and warm water per rectum, after which administer Dr. Korinek's Diarrhoea Capsules according to directions on the package.

DR. KORINEK'S COLIC CAPSULES are compounded with the idea of removing the cause. They are anti-spasmodic and work directly on the circular fibres of the intestines. They are also a heart stimulant-exactly what is needed in cases of colic.

Beware of colic remedies that are given in drop doses, for they contain drugs that only relieve the pain, they cannot remove the cause-and when the effect has worn off the disease has progressed, the heart is weaker, and the chances are that you will lose your animal. mouth.

Always bear in mind that a horse cannot breathe through the

Never drench a horse, it is dangerous. 


\section{Colic-Wind or Flatulent}

CAUSE: This dangerous form of colic is due to the distension of the bowels from gas. The gas is generally produced by the decomposition of undigested food. Wind or Flatulent colic sometimes follows Spasmodic Colic, in which case there are spasms due to irritation from undigested matter, and subsequently distension owing to its decomposition. As a rule Wind or Flatulent Colic is caused by errors in feeding or watering. In some instances it is produced from giving the animal a large quantity of rank grass or watery roots, which on account of their moist nature, is quickly swallowed before being properly masticated.

SYMPTOMS. The signs resemble those of spasmodic colic, except that they are less violent. In most cases there is a gradual accumulation of gas, the abdomen is distended to a considerable size before the animal shows signs of uneasiness. In cases where the animal swells on the right side, it is the large intestines that are filled with gas. In other cases where both sides are equally swollen the stomach and small intestines contain gas. The horse's back will have an arched appearance, passing gas from the anus frequently, the horse will make attempts to vomit. In some cases vomiting actually takes place. This is a bad sign, as rupture of the stomach usually occurs at this stage.

TREATMENT. Give one of Dr. Korinek's Physic Capsules. Rub the abdomen briskly for half an hour, then apply blankets wrung out of hot water. Injections are very beneficial. Use one gallon of water with about two ounces of turpentine added. Also give one of Dr. Korinek's Colic Capsules every hour. Puncturing the intestines is advisable in some cases. This requires a special instrument and no one should attempt the operation without knowing the anatomy of the part, as the artery of the intestines may be injured and produce an internal hemorrhage or an infection may be set up producing abscesses.

\section{Cold-Nasal Catarrh}

CAUSE. Atmospheric changes common in the Spring and Fall, animal allowed to chill when standing in a draft, or driven when the system is in a poor condition. It is also produced by inhaling irritating gases, smoke, drenching through the nose, feeding dusty hay or grain that contains infectious matter.

SYMPTOMS. Animal is stupid, does not take food very freely, hair stands and looks dusty, throat becomes sore, pulse is not greatly affected. There may be a slight rise of temperature, say 101 to 103 degrees F. After a day or two there will be a discharge of mucous from the nostrils which may be offensive to the smell. There is generally an increased flow of urine. The breathing is not much affected. 
TREATMENT. Make the animal comfortable by placing in a clean, dry stall, where plenty of pure fresh air may be had. Avoid all drafts. Give one teaspoonful of Dr. Korinek's Distemper Remedy three times a day, placing it on the animal's tongue with a wooden paddle. Treatment is very simple if administered in the first stages, but if relief is not given, ailment becomes chronic and terminates in Nasal Gleet.

\section{Corns}

CAUSE. Dry feet, increased pressure from ill-fitting shoes, or high-heeled shoes, which tend to contract the heels and produce corns. Wide flat feet are predisposed to bruises which terminate in corns.

SYMPTOMS. Lameness, or as the old saying goes, "the animal will go tenderfooted." When standing the animal is generally very restless, they paw their bedding behind them at night. Tapping or pressure on the foot will assist in locating a corn.

TREATMENT. Discover the cause and remove if possible. Take away all pressure from over the corn and turn animal out in some damp pasture. If this cannot be done put on a flat or "bar" shoe, packing the sole of the foot with Dr. Korinek's Hoof Remedy, then place a leather between the foot and shoe. Also apply the Remedy to the whole of the hoof. Continued applications of Dr. Korinek's Hoof Remedy to the sole and wall of the feet every week will keep them soft and flexible, and with proper shoeing your animals will never have corns. Frequently coffin-joint lameness or navicular disease is mistaken for corns.

\section{Coughs-Acute and Chronic}

A cough is a symptom of various diseases, which should be treated in addition to the cough itself.

KINDS OF COUGHS. Many writers give several different varieties, but for the sake of convenience I will divide them into two classes, namely: ACUTE AND CHRONIC.

CAUSE. Acute Coughs are generally due to sudden exposure to cold, drafts and are the forerunning causes of the diseases of the organs of breathing.

CHRONIC COUGHS are associated with and are often the result of sore throat, lung fever, pleurisy, bronchitis, broken wind, influenza, nasal gleet, catarrh, glanders, heaves and distemper.

TREATMENT. Use Dr. Korinek's Distemper Remedy as directed on the package. Turn the animal out to pasture if the weather is not too cool, dampen the hay or grain which is fed, as dry, harsh or dusty foods tend to aggravate a cough. 


\section{Cracked Heels}

CAUSE. There is little doubt in my mind that ammonia, which is so plentifully found in ill-kept stables, is the chief cause of cracked heels. The action of ammonia on the skin is to render it soft and pulpy, and diminishes its strength by separating the layers of which it is composed.

SYMPTOMS. When inflammation is set up in the part, the secretion of oil is interferred with and cracks occur when the pastern joint is bent where the skin has become wrinkled. The discharge from cracked heels has an offensive odor. In early stages there is extreme heat and swelling; there is also pain and lameness which will disappear as the case becomes chronic.

TREATMENT. Keep the affected parts as dry and clean as possible and apply Dr. Korinek's Hoof Ointment morning and night after washing with castile soap and warm water. Feed carrots and green grass, and if possible, hot bran mashes every morning. Sometimes it is well to give a physic, such as Dr. Korinek's Physic Capsules. It reduces the swelling of the legs and tends to thin the blood.

\section{Curb}

CAUSE. Faulty conformation of the hind legs, that is to say, if an animal has crooked legs, a slight sprain from slipping or jumping will produce Curb. In cases where an animal has well proportioned limbs and is afflicted with Curb, it is caused by a rupture of the small ligament or cord situated just back of the hock.

SYMPTOMS. A swelling will be noticed on the back part of the hock. At first the animal is lame and the enlargement is hot and painful. After a few days' rest the inflammation will partially subside and the enlargement can be plainly seen. When the animal is walked about he may be very lame at the start, but this will disappear as he is moved.

TREATMENT. When the Curb is hot and painful it is well to apply ice packs or cold water to the part. When the inflammation subsides apply Dr. Korinek's Absorbent Blister and rub it in well for twenty minutes; repeat 48 hours later.

If the Curb is of long standing it is more difficult to treat, in which case the above treatment should be repeated again in two or three months. Do not use the animal in drawing heavy loads or drive on slippery roads for two or three months until the blister has strengthened the ruptured tendons. 


\section{Distemper-Strangles}

CAUSE. Distemper is placed among the germ diseases and is produced by the Streplocussus of Schutz. It is contagious and a number of animals in the same stable may become affected at the same time. It is supposed to attack an animal but once, but it may be contracted a second time. May occur at any time of the year.

SYMPTOMS. The animal will first appear dull and show loss of appetite and the hair will look dull and rough. There will be a watery discharge from the nose, and in a day or so a lump will appear between the jaws; the animal keeps his head in a peculiar position; saliva runs from his mouth; the pulse will be a little faster than normal. The breathing will become more rapid and the lump between the jaws will get larger. This lump, or tumor, may form in other parts of the body, on the shoulders, in the groin, lungs or intestines. It usually causes death if it cannot be absorbed. This is called irregular distemper. A determined effort should be made to draw the lump or tumor to a head as soon as possible.

TREATMENT. Place the horse in a clean, comfortable stall, excluding all drafts; blanket the animal, hand rub the legs and bandage; give inhalations of steam from hot water and turpentine; this will relieve the hard breathing. Remember, a horse cannot breath through his mouth, therefore, liquid drenches are dangerous. Give one teaspoonful of Dr. Korinek's Distemper Remedy every four or six hours according to the severity of the attack. Dr. Korinek's Distemper Remedy is in the form of a thick sticky paste which can be placed on the tongue of the animal, where it gradually dissolves, lubricating the air passages for two or three hours or even longer. To assist the lump or tumor in coming to a head apply a blister or liniment, preferably Dr. Korinek's Liniment, applied three times daily. If you fail to draw it to a head in three or four days it is best to secure the services of a good veterinarian who will lance it.

After an attack of Distemper the animal is usually very much run down. Feed Dr. Korinek's Stock Tonic and see him gain in flesh, looks and spirit.

\section{Diarrhoea}

CAUSE. Sudden change of food, frozen food, soft food, unwholesome food, stagnant water or drinking a large quantity of water at one time, purgative medicines, or it may be associated with blood diseases, lung and intestinal affections, or produced by micro-organisms. Many horses, particularly slack loined, slight, "washy" animals, purge if worked or excited, as may be observed among race-horses when taken to a race course. 
Diarrhoea may also be due to worms or it may be merely an effort on the part of nature to expel some irritant matter from the bowels or from the blood, in which case it should on no account be prematurely checked.

SYMPTOMS. Frequent loose evacuations of the intestines, with or without pronounced abdominal pain; generally, loss of appetite, animal looks gaunt and the hair rough.

TREATMENT. Keep the animal quiet, warmly blanketed, and comfortably stabled. Give pure water, often but in small quantities, feed moderately on clean food, such as, rolled oats and dry bran. Give one of Dr. Korinek's Diarrhoea Capsules every eight hours; they will not only check the diarrhoea but will tone the muscular fiber of the intestine which aids in throwing off waste or irritant matter from the system. If the horse shows signs of colicky pains, give Dr. Korinek's Colic Capsules as directed. Animals that are subject to diarrhoea should be fed Dr. Korinek's Stock Tonic which facilitates digestion by stimulating the flow of gastric juices.

\section{Dropsy of the Belly, Chest, Sheath, Udder and Legs}

CAUSE. Poor circulation. Kidneys not working properly, lack of exercise, diseases of the lungs, liver, heart, womb or sheath. Mares heavy with foal often have dropsical swellings.

SYMPTOMS. Swelling seldom contains fluid, although sometimes a sticky serum oozes through the skin; fingers pressed against the swollen parts leave impression.

TREATMENT. Avoid giving physics in this condition when possible, especially to mares with foal. Feed laxative food, such as hot bran mash, green grass, carrots, potatoes, etc. Dr. Korinek's Kidney Capsules are wonderfully effective in dropsical swelling and if given twice a day, will bring desired results within a few days.

\section{Eczema}

CAUSE. Anything that interferes with the healthy action of the skin as, checked sweating, irritation from dirty blankets or harness, or from accumulation of dirt on the skin through want of grooming, errors in feeding, overheating, or by infection. In some cases the cause seems to be constitutional; in others, local. Though the disease is not parasitic in character, it is probable that when once contracted, the diseased parts may become infected.

SYMPTOMS. Slight dryness and eruptions that may affect the head, ears, neck, shoulders, flanks, inside of thighs and root of the tail, followed by vesicles or pimples which may burst and discharge or the contents may be absorbed. The animal will rub 
against the stall, manger, or any other object he can reach, until the parts are very sore, or if worked, he will rub himself violently when unharnessed.

TREATMENT. Give one of Dr. Korinek's Physic Capsules to move the bowels freely. Feed as much easily-digested food as possible, such as carrots, apples, grass, hot bran mashes, steamed rolled oats, etc. Dr. Korinek's Stock Tonic is a blood purifier and is especially adapted for the above-mentioned malady.

\section{Eye Diseases}

CONJUNCTIVITIS, or Inflammation of the superficial structure of the eye.

CAUSE. Direct or indirect injury to the eye as, a blow from a whip, dust, sand or chaff in the eye, or it may be due to extreme cold, heat or foul air.

INFLAMMATION OF THE MEMBRANE OF NICTITANS. The membrane of nictitans is an accessory eye-lid common to all domestic animals, the purpose of which is to remove foreign substance from the eye in much the same manner as we use the hand.

SYMPTOMS of conjunctivitis or inflammation of the membranes of nictitans are very much the same. A partial or complete closure of the eye and a watery discharge due to over-stimulation of the lackrymal glands, the fluid being secreted so abundantly that it is impossible for the tear duct to carry it away; hence, there will be a continuous overflow of tears down the horse's face. The formation of a film or scum over the eye need not cause alarm if the eye shows no sign of puncture.

TREATMENT. Examine the eye carefully and remove any foreign body with a clean feather. Apply Dr. Korinek's Eye Remedy three or four times daily. Avoid the use of liquid medicines, as they are hard to apply and the animal throws them out by shaking its head.

\section{Failure to Breed-Barrenness}

CAUSE. Contraction of the neck of the womb, growths on or in the ovaries, whites or leukorrhea. The first is the only form of barrenness which responds readily to treatment.

TREATMENT. Wash the hands in some antiseptic solution such as carbolic acid, bichloride mercury, and see that the fingernails are smooth. Grease the hand and arm with vaseline and proceed to dilate the neck of the womb. It may be difficult at first to insert one finger but the opening will gradually enlarge. Work slowly and carefully until three fingers may be inserted. Breeding should follow about three hours after the womb has been dilated. 


\section{Filariae-(Thread-like Worm)}

CAUSE. Drinking stagnant water or eating hay gathered from swamps or marshy land. When full grown, the worm measures from two to six inches in length; the tail is more or less curved. They are usually found in the lung cavity, the heart sac, and the intestinal cavity from which they sometimes descend into the sac containing the testicles. Occasionally they are found in the watery humor of the eye. Animals said to have a snake in the eye have been exhibited as curiosities; in all cases the simulated snake was nothing more than the Filariae.

SYMPTOMS. Colicky spells, poor appetite, indigestion, potbelly, rough coat, swelling of the sheath, legs, and lower surface of the belly.

TREATMENT. Prevention is the only treatment, for the worms, once they have entered the digestive canal, it is impossible to remove them.

\section{Fistula-Or Fistulous Withers}

Fistula is a solution of continuity to a greater or less extent. In fistula proper there are two openings, one external and one internal, into an inner cavity. If there is but one opening, it is called incomplete, if two openings, it is called complete. There is no particular difference between fistula and sinuses.

CAUSE. An ill-fitting collar or saddle, being struck by or rolling over an object and bruising the withers, sometimes from being bitten by another horse when playing or fighting.

SYMPTOMS. More or less swelling, slight heat. Pressure upon the swelling causes pain. In the first stage there is only inflammatory action. The second stage is suppuration and in some cases there may be great swelling with but little pus formed. In other cases there is excessive suppuration and the pus makes its way out and discharges at the surface, where sinuses are formed extending in various directions; sometimes the discharge becomes chronic and produces an enlargement.

TREATMENT. If Dr. Korinek's Absorbent Blister is applied before the sinuses are formed, they may be prevented from forming, but after the so-called "pipes" form, I advise that you secure the services of a competent veterinarian.

\section{Founder}

CAUSE. Overeating or drinking-in fact any irritation of the stomach or intestines is liable to be followed by founder, owing to the similarity in the structure of the foot, skin and mucous membranes. Horses with weak feet are predisposed to founder but it may also occur in strong-footed animals. Founder is also produced by allowing the horse to chill after a hard drive on a hot or cold day. 
SYMPTOMS. The horse is stiff and moves with great difficulty; he will generally, though not always, remain standing. Throws his weight upon the heel of the foot to relieve the toe, and if an effort is made to back him, he will drag his feet. Excessive heat is present at the top of the hoof and a throbbing of the arteries may be felt. When the fore feet only are affected, the horse will relieve them of as much weight as possible, when walking, by placing the hind feet well under the body, which results in a peculiar jumping motion. Founder may be mistaken for disease of the lungs or kidneys, owing to the standing position and arched back. Veterinarians have been known to mistake it for lung fever; the services of such men are dangerous and should be avoided. Founder may occur in all four feet but the fore feet are more often affected than the hind ones. Mares sometimes founder after giving birth to a colt, due to inflammation of the womb; symptoms correspond to those of common founder.

TREATMENT. In all cases of founder give one of Dr. Korinek's Kidney Capsules every six hours. Find the true cause of the disease if possible. It is well in most cases to give one of Dr. Korinek's Physic Capsules every eighteen hours till there is free action of the bowels. Physics are not recommended, however, where founder follows excessive irritation of the stomach and intestines, or in mares with foal. Feed soft, easily-digested food, such as carrots, kale, apples, potatoes, hot bran mashes, steamed rolled oats, etc. Have the animal stand in mud, water, or apply bags containing mud, bran, ice-in fact anything tha will cool and moisten the parts. Elevate the hind quarters and give rectal injections of warm water and glycerine.

\section{Galls}

CAUSE. Injuries from ill-fitting collars, saddles, harness, hobbles and scalping boots.

TREATMENT. Remove the cause. Never wash a gall with water, as this prevents its healing, nor use oils or salves, as they accumulate dirt, dust and germs which may cause infection. Apply Dr. Korinek's Antiseptic Gall Powder. It heals and refreshes galls and wounds by forming a smooth surface over the part which permits it to heal while the horse works. Apply as directed on the package.

\section{Glanders or Farey}

CAUSE. A specific germ (Bacillus Malleii or Bacillus of Glanders.) This dread disease is very contagious and is transmittable to man as well as to animals. Cattle and sheep, however, are immune. The disease may be contracted at watering troughs, stables, horse-shoeing shops, boats, and trains or transmitted by harness, bits, curry combs, bedding, pails, etc., as well as by direct contact with a diseased animal. 
SYMPTOMS. The animal seems poorly, though the appetite is good at times, loses flesh, sweats easily, temperature is above normal and a cough is usually present; legs and abdomen are swollen; discharge from the nose, often tinged with blood and very sticky; the membrane of the nose looks dusty and, if closely examined, shows ulcers or spots; the glands under and back of the ears and between the jaws are hard and lumpy. Glanders or Farcy also affects the skin; eruptions or nodules called Farcy Buds or Farcy Buttons and varying from the size of a pea to that of a hickory-nut are found on the inside of the hind legs, under the abdomen, on the side of the chest, shoulder, and neck, also around the nose, lips and face. The discharge from these eruptions is of a greenish-yellow color and very sticky. Glanders or Farcy may be mistaken for Nasal Catarrh, Nasal Gleet, Ulcerated Tooth, Nettlerash, Lymphangitis, Distemper, etc. The disease is fortunately not very prevalent in this country, as every precaution has been taken to stamp it out.

If at any time you have reason to suspect an animal has this disease, no matter whether it is your own or a neighbor's or a transient animal, it is your duty to report it at once to the State Veterinarian.

\section{Grease Heel}

CAUSE. Parasitic fungi invading cracked heels.

SYMPTOMS. Offensive discharge from the glands under the skin which, if not properly treated, red spots will appear, and yellow discharge will form a hard crust sticking to the roots of the hair.

TREATMENT. Cleanliness is one of the most important measures, also a good nourishing diet. If the skin is swollen and tender, poultice with hot flax seed or bran. After the swelling and tenderness have abated, wash well with clean water and castile soap and apply Dr. Korinek's Hoof Remedy morning and night. Feed green grass, carrots, kale, apples or potatoes if possible, also bran mashes. In all cases of Grease Heel give one of Dr. Korinek's Physic Capsules. It removes irritants from the bowels and promotes the digestion.

\section{Heaves-Emphysema of the Lungs}

CAUSE. Fast or heavy work. It may follow lung fever or pleurisy, or the animal may inherit weakness in the walls of the air-cells of the lungs. A very common cause is feeding dusty or dirty hay or bulky food. Horses that are accustomed to eating ravenously are often victims of heaves.

SYMPTOMS. Disease may develop slowly or rapidly. When the animal is at rest the air is taken into the lungs in a more or less normal manner, but is expelled by two distinct efforts, the abdominal muscles aiding the lungs in expiration, as may be seen by the 
heaving of the flank; the movement of the ribs in breathing is scarcely noticeable in a heavy horse. A healthy animal when at rest will throw the air from the lungs in a single effort. The difficulty in breathing is constant and increases in proportion to the amount of food in the stomach and intestines. At the beginning of the attack there is a spasmodic cough which is more or less intermittent; this develops later into a short, weak, suppressed cough, as if the animal lacked strength in his chest to give a full cough, and is often accompanied by expulsion of wind from the anus, which is somewhat protruded.

TREATMENT. Feed sparingly on good grain and hay, which should be dampened with water, if dusty. Do not feed musty, dusty, or bulky food, but give plenty of carrots, potatoes, apples, kale and green grass. Dr. Korinek's Distemper Remedy is very soothing to the breathing organs and should be given after feeding. It is placed on the tongue with a paddle or spoon where, being a paste, it adheres closely, lubricating the air-passages and will last from six to eight hours. In cases of long standing give Liquor Arsenicalis-two tablespoonfuls daily in the feed. After a week or ten days the dose should be increased to three or four tablespoonfuls. Have your druggist omit Tr. Lavender.

\section{How to Find the Age of Your Horse}

To tell the age of any horse, Inspect the lower jaw, of course; The six front teeth the tale will tell, And every doubt and fear dispel. Two middle nippers you behold Before the colt is two weeks old; Before eight weeks two more will come, Eight months the corners cut the gum. The outside grooves will disappear From middle two in just one year. In two years from the second pairIn three years "corners," too, are bare. At two the middle "nippers" drop; At three the second pair can't stop. When four years old the third pair goes. At five a full new set he shows.

The deep black spots will pass from view At six years, from the middle two; The second pair at seven years; At eight the spot each corner clears. From middle "nippers" under jaw At nine the black spots will withdraw; The second pair at ten are bright; Eleven finds the corners light. As time goes on the horsemen know The oval teeth three-sided grow; They longer get-project-before Till twenty, when we know no more. 


\section{Horse Dentistry}

This is a very important branch of Veterinary Science although if I were to go into detail on the subject it would require the writing of an individual volume. This science requires considerable practice. The price of special costly instruments that would make it prohibitive for the stockman to attempt doing his own dentistry.

My advice is to secure nothing but the services of a qualified Veterinarian, who has had privileges of a thorough knowledge of Veterinary Science.

The art of animal dentistry has been abused by the owners of stock securing services of irresponsible men in the veterinary profession who do not look to the betterment of the animal's condition, the owner of the animal not being able to see the condition of the animal's teeth for himself, he is talked into having the animal's teeth worked on regardless of whether it is needed or not. Perhaps the veterinarian needs the money and he will pull and crack healthy, sound teeth, also lacerate the poor animal's mouth. Be sure the veterinarian employed is reliable.

\section{Influenza; Pink Eye; Epizootic Catarrh}

CAUSE. Influenza is a specific and infectious fever which shows a marked tendency to rapidly spread over a large area of country. It appears suddenly without preliminary symptoms, and may become fully developed in twenty-four hours.

SYMPTOMS. The usual symptoms are those of catarrh, although bowel, lung and brain complications may be present, either single or combined. It always gives rise to great weakness. The distinguishing characteristic of influenza, from distemper, sore throat and other diseases affecting the organs of breathing is the suddenness of the attack, rise of temperature, varying from $103^{\circ}$ to $106^{\circ} \mathrm{F}$., pulse feeble and fast and a pinkish, swollen appearance of the inside of the eyelids. The animal is dull, in some cases almost unconscious. Sometimes the legs are very stiff and swollen and there is great difficulty in moving about.

TREATMENT. Pure fresh air, avoid drafts, pure water to drink in small quantities, but often. Blanket the animal, hand rub and bandage the legs. Give Dr. Korinek's Distemper Remedy, one teaspoonful on tongue every four hours. When temperature is high give Dr. Korinek's Fever Capsules as needed. Do not give physics when the lungs are affected, as this is dangerous. Give soft food, such as hot bran mashes, steamed oats, carrots, apples, potatoes, or green grass. 


\section{Lampas}

CAUSE. In young horses it is commonly caused by cutting teeth. In older animals it is usually due to indigestion.

SYMPTOMS. A puffy swelling and redness of the gums. The animal may have difficulty in eating.

TREATMENT. In young animals when cutting teeth, let nature take its course, but when treating a horse of four years or more, give one of Dr. Korinek's Physic Capsules. Then tone up the digestive organs by feeding Dr. Korinek's Stock Tonic as directed on package.

\section{Leeches (Haemopis)}

The leeches which suck the blood of the horse may be divided into two classes: the external parasites which attach themselves to the skin of the legs and adjacent parts of the horse, and the Haemopis Sanguisuga and others of this class which, not being able to penetrate the skin, endeavor to enter the mouth or nostrils of the horse when he is drinking or grazing in wet and leechinfested pastures. They sometimes cling to the mucous membrane of the eyes. The horse leech, which lives in the water, usually gain access to the mouth and nostrils of the animal when young and not more than one-tenth of an inch long. They rarely go beyond the air and food passages, generally fastening themselves to the walls of the windpipe and gullet, where they cling till the animal dies from loss of blood or suffocation. They often cause bleeding from the mouth and nostrils and may be seen by close examination.

TREATMENT. Remove the parasites and sustain the strength of the animal by suitable food. Give Dr. Korinek's Stock Tonic and also feed common stock salt. Where the leeches cling very tightly to the mucous membrane of the mouth and nostrils, it is well to cause the horse to inhale the vapor of hot water containing turpentine.

\section{Lockjaw-Tetanus}

CAUSE. The bacilli of Tetanus are widely distributed and can be found on practically every part of the globe. Their favorite place of production, however, is in barn yards and marshy ground. They are frequently swallowed by stock along with forage, and can often be seen in recently expelled feces. The most favorable temperature for their development is about $70^{\circ} \mathrm{F}$. They act by means of extremely virulent poisons which they produce, and which cause the terrible symptoms that are characteristic of the disease.

SYMPTOMS. The muscles of expression are usually the first brought under the continual spasm of tetanus, and when thus affected give the face of the animal a pinched and drawn in appearance. The other muscles of the head and those of the neck, are 
next attacked. The mouth is closed, the nose poked out, the head elevated. The muscles of breathing and those of the limbs become contracted so that the neck is hollowed, and the tail is raised; the horse stands with outstretched limbs. The animal shows great stiffness or rigidity in attempted movenents. The eyes are sunken and when startied, or excited the breathing is quickened and the flanks have a wrinkled or corrugated appearance. Death may quickly occur from continuous spasms of the muscles of the throat. Another sign is the flying up of the accessory eyelid when the animal is excited.

TREATMENT. If noticed in its first stages and if the animal is able to eat, secure the services of a good veterinarian and insist that he use fresh tetanus vaccine. This disease, however, is almost impossible to cure. Ninety per cent die.

\section{Lung Fever-Pneumonia}

CALSE. Predisposition is largely accountable for this disease, which is more common to young horses than old; also, changes of temperature, introduction of foreign bodies or liquids into the trachea (windpipe) and the bronchial tubes, inhalation of smoke or irritating gases, excitement, exposure to cold after clipping, turning out to pasture from a warm stable, or injury to chest or ribs from being struck with a pole, etc.

SYMPTOMS. I)ullness in spirit; animal usually shivers or trembles, when this ceases the temperature rises to perhaps 103 or 106 degrees $\mathrm{F}$; the ears and legs grow cold and the mouth hot and sticky; pulse increases to 60 or 90 per minute, full and bounding; breathing short and labored, and abnormally quick, increasing to perhaps 50 inspirations per minute, whereas in health it does not exceed 12 or 13 per minute. A cough is also likely to be present and the animals remain standing until they are on the road to recovery or until death takes place. Other symptoms are, constipation, feces covered with mucus or slime; urination frequent, scanty and dark in color; appetite poor but thirst great; the eyes look glassy and the membranes have an inflamed appearance. It is a good sign if the animal looks about freely. When the critical stage is past the temperature and pulse gradually fall, the appetite returns, the urine becomes more abundant and takes on its natural color, the cough loosens and the discharge from the lungs is profuse and of a yellowish color, and the breathing becomes normal.

TREATMENT. Good care is of the utmost importance. Place the horse in a comfortable, well-ventilated stall, being careful to exclude drafts; hand rub the legs and bandage with woolen cloth; blanket the animal, give plenty of bedding and keep pure water before him at all times. 

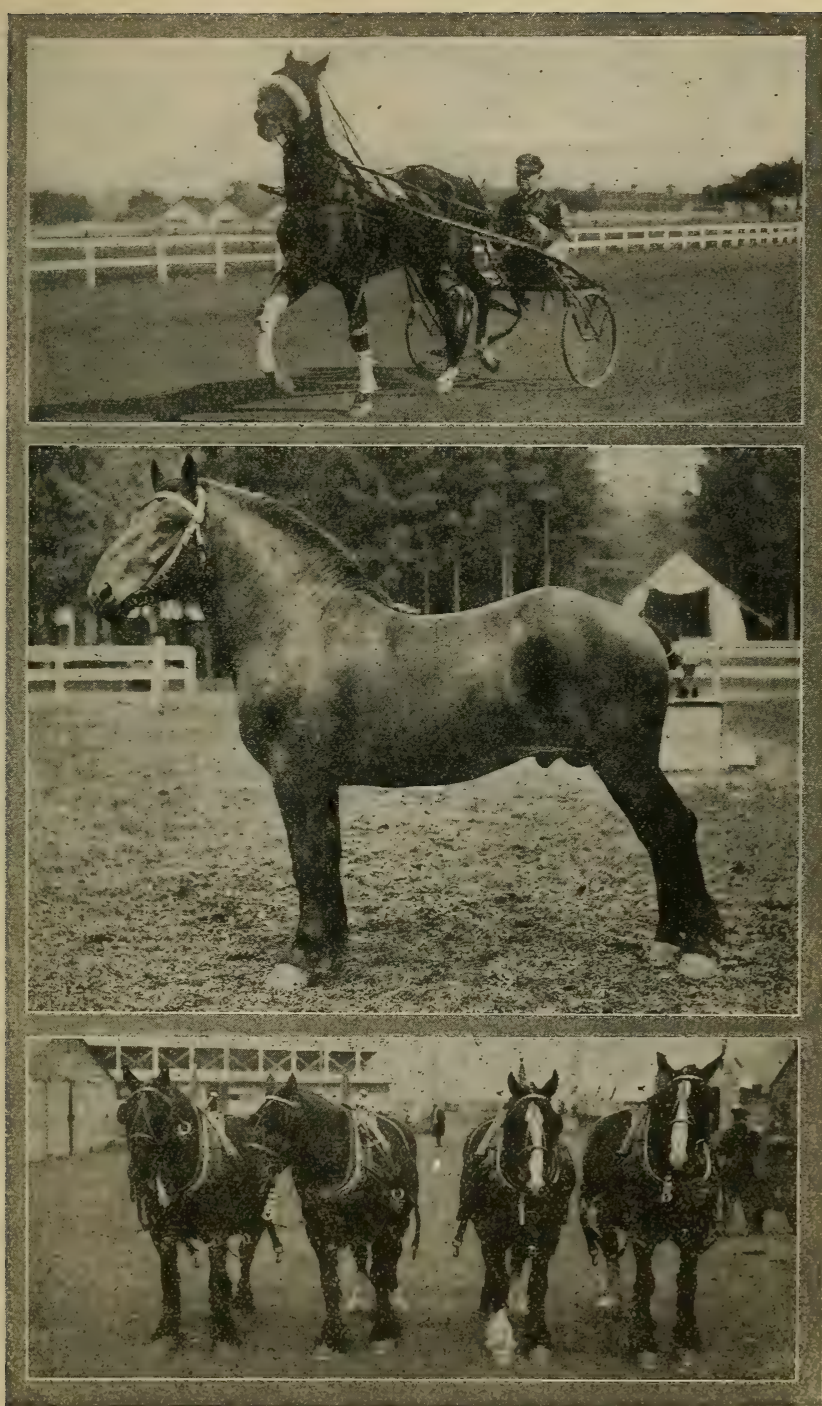

PRIZE WINNING HORSES 


\section{Mange (Scabies)}

CAUSE. Mange is a contagious disease produced by the, presence of a small parasite that varies in length from a fiftieth to a hundredth of an inch, according to the species, of which there are three: Sarcoptes, which generally affects the withers; Symbiotes Communis, affecting the legs; and the Psoroptes Communis, which affects horses about the root of the tail and mane. The latter, especially, is the one most commonly found affecting horses. They multiply rapidly and are spread from diseased to healthy horses by their bodies coming in contact with one another or by corrals, stables, railroad cars, etc., recently occupied by mangy horses.

SYMPTOMS. The mange mite attacks the skin and produces a thickness of its outer surface, covering it with crusts and scabs with a consequent loss of hair. Intense itching accompanies the disease and affected horses continually bite and rub themselves.

Psoroptic mange commences at the root of the tail or at the roots of the mane on the neck or withers and gradually spreads over the back, up to the head, over the sides and finally affects the entire body. In cases of long standing the skin becomes ulcerated, the animal becomes greatly weakened, emaciated and finally dies.

TREATMENT. When a large number of horses are affected in one locality it is best to prepare a vat and dip them, under the supervision of the United States Bureau of Animal Industry. When just a few horses become affected, the following has proven very effective: Sulphur eight ounces, Oil of Tar eight ounces, Sweet Oil two quarts. Mix and apply liberally to the parts affected. A few applications are generally sufficient to eradicate the disease.

\section{Monday Morning Disease-Lymphangitis}

CAUSE. This ailment is common with hard-working horses and is caused by confining them in the stable and allowing their usual amount of food. More nutriment is consumed than can be taken up by the system, which causes an irritation. It is frequently found in certain stables on Monday morning, hence its nameMonday Morning Fever.

SYMPTOMS. Swelling and lameness usually affecting the hind leg inside of the thigh and extending down the leg in a hard ridge. It will pit on pressure and cause intense pain; the horse will have difficulty in extending the limb forward, the swelling may surrouna the leg entirely. Pulse will be 50 to 60 per minute, temperature $10^{2}$ to 104 degrees $F$., breathing will be faster than normal. The animal has great thirst, but the appetite is very poor, usually remains standing, if he lies down will have great difficulty in getting up. 
TREATMENT. Apply hot applications to the affected limb or limbs for one hour, then rub dry and apply Camphorated Liniment. Give one of Dr. Korinek's Kidney Capsules three times daily until the animal is on the road to recovery. Feed soft, easily digested foods, such as hot bran mashes, steamed oats, carrots and green grass if possible.

The parts may regain their natural appearance, but there is a tendency to leave the limb enlarged.

\section{Mud Fever}

CAUSE. Horses driven over muddy roads during the day and exposed to freezing temperature at night, or driven over muddy roads, then washing the limbs and not drying properly, often produces a superficial inflammation of the legs.

SYMPTOMS. The legs are swollen, extremely hot and tender, the horse is stiff, the hair comes off the legs easily and if the cause is not removed, severe complications may follow, as the secretions of the skin become greatly affected.

TREATMENT. Prevention. Horses that are driven over muddy, wet roads should have their legs rubbed dry when stabling them for any length of time. When the legs are badly swollen wash them with clean warm water and castile soap and dry them well with a clean soft cloth. Then apply Zinc Oxide Ointment or a lotion made from Acetate of Lead one ounce, Zinc Sulphate one-half ounce. Place in a quart of clean water and apply twice daily. Either application is very beneficial in the treatment of Mud Fever. Feed the animal wheat bran mashes, steamed rolled oats, vegetables, etc., as they have a good effect on the system which aids in relieving the inflammation of the skin.

\section{Nasal Gleet-Chronic Catarrh}

CAUSE. Exposure to cold followed by neglect, and lack of nourishing food; bruise or fracture of the frontal bones of the head; injury to the blood-vessels inside the bones, or an ulcerated tooth. May also be caused by tumor, or foreign substance or liquids in the nasal cavities. Sometimes dried pus in the nostrils, resulting from a cold, will cause nasal gleet.

SYMPTOMS. A white or yellowish discharge from one or both of the nostrils, the quantity varying with the severity of the attack and the length of time the disease has been established. If, when tapping over the nose below the eye, a dull sound is produced, it is safe to conclude that the cavities are filled with pus; to make certain, compare the sick animal with a healthy one; in some cases you will notice that even the bones of the nose below the eye are slightly elevated. The lining of the nose may be of a red or yellow color but not ulcerated in spots, as in glanders. The animal may 
continue in pretty good spirits and work well for a time, but as the case develops, he becomes lean in flesh and, what is termed hidebound. Always examine the teeth. In a case of long standing the discharge has a fetid smell, differing in this respect also from glanders.

TREATMENT. If not due to injury or an ulcerated tooth, the animal will in most cases recover with proper medical treatment. Give one of Dr. Korinek's Tonic Capsules three times a dáy; if a cough is present, give one tablespoonful of Dr. Korinek's Distemper Remedy every six or eight hours after feeding. When due to injury, tumors, ulcerated tooth, fracture of the bones of the head, or dried pus in the nasal cavities, it is best to secure the services of a skillful veterinarian and one whose professional knowledge renders him thoroughly competent.

\section{Navel String Infection-Umbilical Pyaemia}

CAUSE. While the unborn foal (fetus) is in the womb of its mother it is surrounded by enveloping membranes which constitute the after-birth on delivery. These membranes are attached to the wall of the womb and are connected to the fetus by means of the navel-string (umbilical cord) which is provided with two arteries and a vein for the nourishment of the young creature and for the removal of its waste products.

It also has a narrow canal (the urachus) which serves to remove the urine of the fetus; in fact the subsequently formed bladder takes its origin from a dilation of the urachus. Under normal conditions when the foal is born respiration takes place, the umbilical arteries and vein become quickly blocked up, urine is discharged through the urethra (which communicates with the penis or vagina as the case may be) the foal enjoys a separate existence and the wound caused by the division of the umbilical cord leaves a scar which is known as the navel.

It is usually supposed that the germ of a navel-string infection gains admittance into the body through the exposed surface before the wound is closed. However I am of the opinion that the mother is the bearer of the infection in a great many cases, for in the uterine secretions of mares whose foals fell with navel-string infection, the same characteristic germs were found as were present in the joints of the affected foals. The infectious material is, by the act of covering, conveyed from mare to mare so that the mucous membranes of the womb becomes the habitat of the specific germ.

By the inoculation of these germs into the blood stream of foals an illness has been produced which in the smallest particular could not be distinguished from that arising in naturally affected foals. If the infected germs are transmitted by the mother it is a strange fact that their presence does not produce any disturbance in her. 
This is a very common malady in most places. I have known several instances on particular farms where they were unable to raise either foals or calves, but if the mothers were removed to another farm immediately after or before foaling, the foal or calf lived and was reared without difficulty, and although constitutional debility plays an important part the presence of specific germs constituting an infected area is, I believe, the most important factor in producing this disease.

According to my observation about 75 per cent. of the cases die within the first three weeks after birth. This high rate of mortality would be considerably diminished if proper treatment was adopted.

SYMPTOMS. The attack usually comes on during the second or third week after birth and almost always before the closure of the navel opening, which in affected animals will be found to be in a wet and suppurating condition. Occasionally foals two or three months old which have the urachus closed and are in an apparently healthy condition contract this disease in a form of painful swelling of the joints. The first symptoms are generally dullness; more or less fever; lameness which is often attributed to rheumatism or to injury caused by the mare treading on the foal; and disinclination to move or even to stand. Upon examination the patient will be found to have a soft, gelatinous swelling of one or more of the joints of which the hock, elbow, fetlock, stifle and hip usually manifest the enlargement most clearly.

These swellings are hot and painful to the touch; they tend to suppurate and frequently cause intense lameness. In very rare cases open urachus may be without any joint inflammation. In this disease inflammation of the joints and open urachus are almost always co-existant. Animals which recover from a bad attack are seldom worth the trouble of rearing because as a rule their constitution becomes permanently impaired and one or more of their joints become stiffened by the attack.

TREATMENT. In the treatment of this disease we have to attend to constitutional disturbance, inflamed joints, open urachus and complications such as constipation and diarrhoea.

The comfort of our little patient must be studied under all circumstances. If the weather be at all cold it should be covered with a warm sheet. Should the foal have any difficulty in rising from the recumbent position an attendant should be told off to assist it to rise and see that it is regularly fed. It is only in extreme cases that the foal refuses to suck its dam. During warm weather, and especially if the ground is dry, such a patient is always better off for a little sunshine, but on no account must it be left out during extreme heat as in this state it is very liable to sunstroke. The best food for the mare is grass, which, during the day, she can generally have. The inflamed joints of the foal should be rubbed lightly with Dr. Korinek's Absorbent Blister every 48 hours, which, when applied to the skin, appears to have a well- 
marked antiseptic action on the underlying tissues. An inflamed joint should on no account be bathed with warm water, fomented or poulticed because the application of moist heat would be the best possible means for promoting the development of the infective germs which are the cause of the locai and general disturbance. The open navel-string should not be ligatured because that operation is generally followed by an increased inflammation of the part, and by an aggravation of the other symptoms apparently on account of this outlet for deleterious products becoming blocked up. If the navel string has been ligatured and is in an inflamed state the ligature should be removed without delay.

As a supplement to the food we may give brown sugar or treacle, both of which are easily digested and are very nourishing. Four or five eggs daily will also aid in keeping up the strength.

\section{Navel Rupture-Umbilical Hernia}

CAUSE. Hereditary predisposition is well marked in this complaint. It may exist at birth but, so-called congenital rupture may very probably be the result of the pulling which the navel-string underwent at the time of foaling. However it may be, umbilical hernia usually occurs during the first two or three months after birth; that is to say while the opening at the navel is becoming obliterated and the tissues at that place is becoming consolidated. They can, however, appear later and may result from more or less violent strains occasioned when the foals are jumping or playing. At other times these strains are induced by intestinal irritation accompanied by diarrhoea or constipation with straining. However the strain may take place, the abdominal muscles contract and push the intestines towards the wall of the belly. Then if they find an opening or even a weak spot, like the ring of the navel while it is undergoing the process of becoming closed, they select it and a rupture is produced.

SYMPTOMS. The rupture, the location of which clearly shows its character, may vary in size from that of a hen egg to that of an ostrich egg. If pressed upon with the hand, especially if the animal is placed on its back, will disappear, to return, however, when the pressure is removed. If it be composed of intestines it will be soft and elastic when the bowels are empty, but when they are full of semi-solid food, they will be doughy. In any case the tumor will feel elastic when composed of intestines and when formed of its connecting membranes will naturally not vary in consistence. If intestines be present, movements and abdominal rumblings may be detected in it. This rupture rarely gives rise to serious consequences because its contents are composed of large intestines and omentum, either of which are, in this position, liable to become strangulated. It may, however, become engorged and inflamed from injury. Its existence naturally depreciates the value of an animal suffering from it. 
TREATMENT. In the majority of cases the rupture will disappear of its own accord in two or three months. In case the rupture shows no signs of diminishing in size it is well to apply a bandage around the abdomen. Write our consultation department and we will prescribe a treatment that will apply directly to your animal's particular case.

\section{Open Joint}

CAUSE. Injuries, such as a kick from a sharp shoe, wire cuts, punctures from snags, or from probing a wound near a joint. Open joint is one of the most serious accidents that may happen to a horse for the sufferer is apt to die from the ensuing constitutional disturbance, and even if he recovers, the joint will, in all probability be permanently stiff.

SYMPTOMS. If the joint is opened or severely injured the wound will have an ordinary appearance except that there may be a flow of joint oil from the injured oil sack. However, the discharge gradually becomes more unhealthy until finally it is mixed with pus and blood and assumes a fetid odor. After two or three days the joint swells and becomes very painful and a high fever sets in. In unfavorable cases the animal dies from exhaustion very shortly, or at best recovers with a permanently stiff joint.

- TREATMENT. Never probe a wound near a joint. If the injury is small and is noticed at once, apply Dr. Korinek's Absorbent Blister. This will set up sufficient inflammation to close the opening and kill any infection that may be present, as it possesses powerful antiseptic properties. If the wound is large, wash with some antiseptic such as Dr. Korinek's Dip-one teaspoonful to a pint of water or carbolic acid in the same proportion; the dip is just as effective and much cheaper. The wound should be washed twice daily and bandaged with clean absorbent cotton and bandage. Do not attempt to syringe into an open joint or some of the solution may gain entrance into the joint. Keep the animal quiet and feed on soft laxative food.

\section{Palisade Worm (Strongylus Armatus)}

This parasite thrives on poor and marshy ground and is commonly found in the United States and Canada. The body of the worm is gray in color, more or less stiff and straight and thicker in the front than in the hind part; it varies in length, the male measuring from three-fourths of an inch to one inch and the female from one to two inches. It may occur in an adult or an immature state. In the former it implants itself on the mucous membrane of the large intestines by means of its armed mouth, while in the latter it lives in cysts underneath the mucous membrane of the intestines 
and is sometimes found in the brain, testicles and liver. The immature worms, which do not issue directly from the cysts, get into the arteries and are carried by the force of the blood to all parts of the body.

SYMPTOMS. Same as in Red Worm with the exception of colicky pains caused by the worms blocking the arteries which carry blood to the intestines, thus interfering with the process of digestion. Where the worms enter the arteries of the limbs, it results in lameness. It is a good plan to examine your animals once or twice a year to insure them against this pest.

TREATMENT. Same as for Red Worm.

\section{Petechial Fever (Purpura Haemorrhagica)}

CAUSE. Constitutional weakness following some debilitating disease such as Distemper, Pink Eye, Catarrh and even following operations when an animal becomes weak from want of exercise, in which case it generally appears during his recovery. It is not infectious and cannot be transmitted by inoculations.

SYMPTOMS. There is a slight swelling of the limbs, more likely to be about the hocks. The swelling may disappear by exercising but will soon return. The swellings present a very abrupt appearance, nearly the same as if a string was tied around the limbs and swell very quickly which is symptomatic of Pupura. Exudations take place in which, if on white limbs, you will see little red spots, from which a liquid is oozing. The swelling is very painful and the entire limb may be swollen. Small vesicles appear on the limbs and also on the mucous membranes, and it is well to look at the mucous membranes before forming your opinion, as you will, no doubt, detect these spots, which may extend into the lungs. These spots increase and may run into each other. The mucous membranes of the nose may become a mass of corrupt matter. The upper lip may hang pendulous, which is due to the want of nervous stimulus. If the nostrils are swollen very badly there is difficulty in breathing, the animal is not able to take food, the symptoms are considered very bad. The pulse varies much in some cases; althougn the swelling is very great, the pulse may not be more than forty or fifty per minute. The temperature is elevated one to three degrees above normal, there may be a coughing and a brownish colored discharge from the nostrils. The mouth and eyes become affected and together with the discharge from the nose, the horse is a loathsome object. In milder cases the appetite is retained, or the animal may take food one day and the next refuse it. The bowe's are constipated as a general thing in the first stages of the disease and the urine may be of a dark color, may even contain blood. There may be a peculiar dropsical swelling of these petechial spots or it may show itself in connection with the eyes and there may be blood extravagation without outer symptoms. This disease may 
affect the bowels, liver, lungs, etc. The animal usually stands, perhaps from the difficulty in moving the limbs. It is necessary to watch the case closely, for flies will attack him and he will be filled with maggots. Sluffing may take place; the entire sheath or patches upon the body may sluff off and there may be paralysis of the penis.

TREATMENT. Place the animal in a clean, light, comfortable stall. If the weather is cold, blanket. The following medicine is recommended because of its particular affect on the blood in this disease: Chlorate of Potash eight ounces, Iodide of Potash eight ounces, Quinine Sulphate eight ounces. Make into thirty-two capsules and give one capsule every six hours. Also administer one ounce capsules filled with Spirits of Turpentine three or four times a day. Moisten the capsules with sweet oil and give with capsule gun. Feed hot bran mashes containing two to three ounces of pure flaxseed meal. Also, feed vegetables, green grass, if possible.

\section{Pin Worm; Thread or Maw Worm (Oxyuris Curvula)}

This worm when full grown is about one and three-quarters inches in length; its tail is thin and whip-like, the head thick and terminating in a curve somewhat resembling the crook of a stick. The presence of these parasites may be detected by a light-yellow substance (the eggs of the worms) which adheres to the skin below the anus. Pin Worms, like Round Worms, frequently come away with the fecus.

TREATMENT. Dissolve four tablespoonfuls of common salt in one gallon of warm water and clean out the rectum and when this has been expelled follow with an injection of two quarts of lukewarm water in which has been dissolved one of Dr. Korinek's Worm Capsules. This causes the expulsion of eggs and worms that cling to the walls of the rectum. The worms sometimes make their way so far forward that it is impossible to reach them with injections; in this case treat as for round worms.

\section{Pleurisy}

CAUSE. Exposure to sudden changes of temperature, confinement in damp, poorly-ventilated stables, wounds that penetrate the chest walls, fractured ribs, heart disease, etc. Also occurs in conjunction with bronchitis, influenza and other diseases.

SYMPTOMS. As a rule the lung on the right side only is affected, although one or both lungs may be affected at the same time. The animal is distressed and uneasy, trembles, affected side is painful to pressure. Breathing is short and quick and the flanks heave, which shows that the animal is trying, as much as possible, through action of the muscles of the abdomen, and not allowing any move- 
ment of the ribs. The nostrils are dilated. There is usually a short, dry, painful cough present whick is repressed as much as possible to avoid irritating the inflamed parts. Often when expelling air from the lungs the horse gives a painful grunt, especially when made to move. Pulse is generally hard and faster than usual. Temperature in early stages may rise to 104 to 106 degrees $F$. If the ear be applied to the affected side a dry, crackling or friction sound can be heard. A groove along the lower portion of the ribs will extend back to the flank. Within two or three days the pulse will be softer and weaker, temperature will fall to 101 or 102 degrees F. and fluids form and the painful short breathing will disappear. The liquids may now undergo absorption, if properly treated and the case terminate favorably in a week or ten days. Frequently large quantities of fluid accumulate in the chest that cannot be absorbed, the breathing becomes weak and rapid and the animal dies from exhaustion.

TREATMENT. Give one of Dr. Korinek's Fever Capsules every four hours. It is very important that you apply Dr. Korinek's Absorbent Blister over lungs and rub in well every 48 hours. It absorbs excessive accumulations of fluid which must be prevented from forming. Dr. Korinek's Absorbent Blister when applied over the lungs stimulates blood circulation, which relieves blood congestion in pleurisy. Do not be afraid of leaving a scar if it is not applied too often. Grease such as common hog lard should be applied and rubbed in well 24 hours after each blister. The diet and means of keeping up the appetite are very important. Coax the animal to eat. In pleurisy give grass, carrots, kale, apples, bran or steamed oats.

\section{Poll Evil}

Poll Evil is so called because it occurs in the region of the poll. It is not a constitutional disease, but comes no doubt from wellmarked causes, as from inflammation set up and involving the bones and muscles in the region of the poll, and perhaps of the larger ligament. Owing to the low vitality of the parts and the action of the nead in taking food, etc., the pus is apt to burrow deep into the muscles.

CAUSE. Direct or indirect injury. A common cause is striking the head against a low doorway or on ill-fitting halter or bridle.

SYMPTOMS. Swelling just back of the ears on one or both sides of the head. The animal stands with the nose out; slight heat in the parts, pain on pressure. In the first stages it is merely inflammatory action. The second stage is suppuration, or there may be great swelling in some cases when there is but little pus formed.

In other cases there is profuse suppuration and the pus makes its way out and discharges to the surface and sinuses are formed, which extend in various directions. Any abscess in this region is called Poll Evil.

TREATMENT. The same as for Fistula or Fistulous Withers. 


\section{Quittor (Fistula of the Foot)}

CAUSE. Injuries. Horses working on rough, stony roads are subject to punctures, pricks, bruises, corns, treads, etc., that end in pus formation which does not get a dependent opening and destroys the tissues in which it comes in contact. Finally it bursts, forms sinuses and pipes, as commonly called, at the top of the hoof.

SYMPTOMS. Extreme lameness, heat, pain and swelling will show themselves about the top of the hoof. As a rule a quittor splits slowly and is more or less painful during the first stages. After the sinus is formed and the pus discharges, the inflammation generally subsides. Its healing process is often delayed due to the diseased portion of the cartlidges inside the horny hoof.

TREATMENT. Apply flaxseed or hot bran poultices to relieve the inflammation and hasten the formation of sinuses or pipes. Then with an ordinary syringe inject the following: Silver Nitrate 10 grains, water one ounce. Inject fifteen (15) to twenty (20) drops twice daily. Keep the foot clean and the animal as quiet as possible. It is very disagreeable, as stated before, and the healing is very slow, but this must be naturally expected, as we are unable to provide the sinuses with good drainage.

\section{Red Worm (Strongylus Tetracanthus)}

The Red Worm varies in length from one-third to three and onequarter inches, and is sometimes white, though it usually appears to be red because of the blood it contains. This parasite is found in all parts of the world. Its favorite haunt is marshy land.

SYMPTOMS. Paleness of all visible membranes, eyes watery and inflamed, swelling of the sheath, legs, and lower surface of the belly; fetid diarrhoea, dullness, debility, emaciation, rough coat, and the presence of worms in the feces. The worms when first passed are bright red in color but after being exposed to the air they turn dark and may easily escape the notice of the casual observer.

TREATMENT. Withhold all food for twenty-four hours, then give one of Dr. Korinek's Worm Capsules. Follow this in four hours with one of Dr. Korinek's Physic Capsules. Feed Dr. Korinek's Stock Tonic liberally.

\section{Ringbone}

CAUSE. Faulty conformation, for example, a narrow or straight pastern joint is considered faulty. Be very careful in selecting a sire when breeding, as faulty conformation is hereditary. Ringbone may also result when young animals are put to work on hard roads or stony pastures sometimes produce ringbone, before the bones have become properly hardened. Other causes are injury to tendons or ligaments, bruised joints, blows, calking, or picking up a nail. 
SYMPTOMS. Lameness will manifest itself when the horse first starts out in the morning; this may become less noticeable or even disappear temporarily as the animal works. They will gradually grow lamer and examination will disclose an enlargement at or around the top of the hoof. This may appear in one or more feet, but the front feet are more often affected.

TREATMENT. One important factor in treating Ringbone is keeping the patient quiet. This can be accomplished by placing the animal in a very narrow stall, carrying his feed and drinking water for a month or six weeks. Appiy Dr. Korinek's Absorbent Blister twice within an interval of forty-eight hours, the first and third week of his standing.

To cure a Ringbone it is necessary to unite the ends of two bones together of the affected joint. I am quite sure that you will agree with me that a fractured bone cannot unite if moved frequently. The same action must take place in healing a Ringbone as that of a fracture. The younger the animal the better are the chances for its recovery, the bones in an old animal being hard and brittle, do not heal so quickly as in the young animal.

\section{Round Worm (Ascaris Megalocephala)}

Resembles the earth-worm somewhat in shape, yellowish-white in color, stiff and elastic. When full grown it varies in length from six to sixteen inches. These worms are usually found in the small intestines, although they sometimes invade the stomach, and when numerous, seriously disturb the animal's health.

SIMPTOMS. The animal's general health is affected as is evident from the morbid state of his appetite, rough coat, pot-belly, liability to colic and slight diarrhoea. Some of these worms are often expelled with the feces. As they increase in number they block up the small intestines, giving rise to colic, and may in time kill the horse. They sometimes cause perforation of the bowels.

TREATMENT. Withhold all food for twenty-four hours, then give one of Dr. Korinek's Worm Capsules; follow in six hours with one of Dr. Korinek's Physic Capsules. If the animal is poor and run down, give Dr. Korinek's Tonic Capsules or Dr. Korinek's Stock Tonic as directed on package. It is a good plan to repeat the above treatment two weeks later to insure the removal of any worms which may have survived the first treatment.

\section{Rheumatism}

CAUSE. Exposure to cold rains, drafts, lying on damp ground when the blood is in poor condition. Also due to over-stimulating food.

SYMPTOMS. Lameness, swelling or soreness which may shift from one place to another, then finally locate in or near one of the joints of the limbs. 
TREATMENT. Take away all grain, feed green laxative foods, such as carrots, apples, potatoes, kale, and good hay. If the weather is warm turn out to pasture, but confine to a good warm stable at night. It is advisable to give one of Dr. Korinek's Physic Capsules for its action on the blood is required. Also feed Dr. Korinek's Stock Tonic. When the swellings are painful apply Dr. Korinek's Liniment as directed on package.

\section{Scrotal Rupture (Inguinal Hernia)}

CAUSE. Abnormal size of the upper ring through which a part of the intestines or its connecting membrane descends into and through the canal leading from the abdominal to the scrotal cavity. There is little danger of strangulation from this form of rupture which may occur at birth and disappear with age. A careful examination should therefore be made of the scrotum before castration.

SYMPTOMS. In most cases this condition is easily detected. The scrotum will be somewhat enlarged. Sometimes the intestines will become strangulated and colicky symptoms appear. When a young male colt shows signs of colic, examine him for Scrotal Rupture.

TREATMENT. The trouble usually disappears with age, although in some cases it is well to operate. Where colicky symptoms are present roll the colt on its back, manipulating the scrotum. Diet carefully.

\section{Staggers \\ (Forage Poisoning; Inflammation of the Brain; Cerebral Meningitis)}

CAUSE. Certain plants or stagnant water have the reputation of producing staggers; frequently seen in the early autumn months when the grass in the pastures become dry and certain forage remains green, which contain toxic principles. These plants are ravenously eaten by horses on account of being green and tender. This is one of the common causes of the disease, although mouldy, indigestible or highly nitrogenous foods frequently produce staggers. This form of staggers is not contagious, although what produces staggers in one horse will produce it in another. In this way several horses may become affected with staggers at the same time. Inflammation of the brain may occur as a complication of some infectious or digestive diseases. Other causes are blows to the head, tumors in or on the brain, which causes the animal to naturally stagger, as the brain controls the horse's organs of locomotion.

SYMPTOMS. Vary to a certain extent, but a careful observer will detect some trouble connected with the nervous system, as the animal walking unsteadily, stepping high and keeping the legs 
spread apart, bracing itself to keep from falling. There is also great depression, as dullness and sleepiness with little or no inclination to move about. The head may be placed against a wall or fence and the legs kept moving as if they were trying to walk. As the disease progresses and no attempts are made to relieve it they will become fractious, nervous, easily excited, pawing and eventually fall, keeping the feet moving as if they were walking, throwing their head about in a delirious manner and death follows. The hor'se as a rule eats and drinks ravenously when the first signs of staggers are noticed, but in its latter stages the tongue and gullet becomes paralyzed and although the animal attempts to eat and drink they cannot swallow. The pulse varies. It is strong, but sub-normal when the first symptoms of staggers are noticed, that is to say, they are as slow as 20 to 25 beats per minute. As the disease progresses, however, they become weaker and faster. Constipation frequently accompanies this disease, also paleness tinged with yellow about the mucous membranes of the mouth and eyes. In many instances I believe that the poisonous forage eaten by horses depresses the heart action to such an extent that it results in the brain not receiving the proper blood supply, causing dizziness or staggers.

TREATMENT. Place the animal in a clean, dark stall, keeping the surroundings as quiet as possible. In its first stage it is easily treated, but as the horse becomes easily excited and his swallowing becomes difficult, it is very unsuccessful. When the first signs are noticed administer one of Dr. Korinek's Physic Capsules. If good bowel action is not produced within twenty-four hours, administer another. This removes the irritant from the intestines and prevents its absurption into the blood. Also administer one of Dr. Korinek's liever Capsules every four hours.

My method of administering medicine to animals places me in pusition to treat them and compel them to take medicine even though paraly'sis may exist. When animals will eat, give food that is tasily digested, as hot wheat bran mashes, steamed rolled oats and vegelables and give small quantities, but often of clean, fresh water. It is necessary to give stimulants and tonics as soon as they are on the road to recovery. Administer one of Dr. Korinek's Tonic Capsules two or three times daily.

\section{Sore Throat (Pharyngitis; Laryngitis)}

CAUSE. Exposure to cold weather or rain when the animal is not accustomed to it; drenching with irritating medicines or inhaling irritating smoke or gases.

SYMPTOMS. At first the animal generally chills, the legs and ears cold, but eventually they become very warm as the temperature increases, coughing, grinding of the teeth, saliva oozing from the mouth; the animal will hold its head in a stiff, straight position, moving it as little as possible. There will be great difficulty in masticating and swallowing, as the food will come from the mouth 
in the form of wads, and as this soreness of the throat progresses food will also come from the nostrils. This is a bad sign, as extensive inflammation is no doubt present. Water, also, runs through the nostrils freely when the animal attempts to drink, due to the swollen condition of the throat. The animal forces the water back into the mouth, but is unable to swallow and hence the water gushes out through the nostrils. The animal evinces great pain when pressure is applied from the outside and they breathe with great difficulty. Although the pulse are not much affected at this stage, the temperature is elevated from one to two degrees above normal. The urine becomes scanty and highly colored, the eyes blood-shot and discharging. Eventually the throat becomes greatly swollen and abscesses may form and discharge. As a rule constipation is associated with this disease.

TREATMENT. Mild attacks of sore throat are easily treated, but when serious cases develop it is unsuccessful. Place the animal in a clean, comfortable stall; permit as much fresh air as possible, but avoiding drafts. If the weather is chilly, blanket the animal, hand rub the legs and bandage with woolen cloths or bandage. Administer Dr. Korinek's Distemper Remedy, one teaspoonful every two hours. In mild attacks give less frequent. When they will eat, feed food that is easily digested, as hot wheat bran mashes and steamed rolled oats, containing two or three ounces of pure ground flaxseed. It is always necessary to apply strong liniments to the throat, as they relieve inflammation and stimulate the formation of an abscess. Apply Dr. Korinek's Liniment. This will be found very beneficial. It will relieve the inflammation and hasten the formation of an abscess. If the swelling is extreme between the jaws, so as to interfere with the animal's breathing, it is well to lance the abscess if a soft spot can be found. Just cut through the skin with a knife; then use a clean, blunt instrument to locate the pus cavity. Otherwise severe hemorrhage may be produced.

\section{String-Halt}

CAUSE. Several theories have been put forth as to the cause of string-halt, and it is generally supposed to be a nervous disease; a condition opposite to paralysis. The exact cause of this disease is hard to determine, but it is likely to occur in highly nervous horses. It sometimes follows an injury which may have irritated tne nerves in some way. I believe that castration causes it in many instances, due to the severe struggle when being thrown, or pulling down severely on the spermatic cord when removing the testicle.

SYMPTOMS. Spasmodic contraction of one or both limbs. This sign varies, as sometimes it is very violent, while in others it may be so slight that it is hard to detect when stepping the horse forward, but on backing or turning the horse around the signs are easily noticed. All symptoms are better marked in the winter than in the summer, as some show it in the winter that do not show it in the summer at all. 
TREATMENT. Very unsatisfactory, although an operation is successful in some cases, but if this is attempted, the services of a competent veterinarian should be secured.

The feeding of laxative foods that are easily digested relieves string-halt in many instances.

\section{Shoe Boil-Ciapped Elbow}

CAUSE. Due to injury or pressure from lying down on a sharp shoe. A rough floor also has a tendency to produce it.

SYMPTOMS. Enlargement of the elbow joint, which becomes tender and painful. When it first appears it is flabby and feels as if it contained fluid. Many veterinarians make the mistake of lancing the swelling.

TREATMENT. Do not lance, but let it come to a head of its own accord, then apply Dr. Korinek's Absorbent Blister every forty-eight hours. Rub in well for about twenty minutes at a time.

\section{Side Bones}

CAUSE. The chief causes of sidebones are: One, deprivation of frog pressure; two, injuries; three, high heeled shoes, the use of which is almost entirely confined to draft horses, a high-heeled shoe prevents the frog from resting on the ground, which is the natural support of the foot.

SYMPTOMS. Enlargement just above the hoof, usually affecting the front feet, or which may affect only one side of one of the feet. The pain which produces the lameness is due to pressure on the soft tissues between th newly formed sidebone and the hoof. Sometimes the cnlargement has a tendency to spread the hoof. In such a case the lameness is not so severe.

.TREATMENT. Clip the hair from the part and rasp the hoof thin just below the sidebones, so that the hoof will dent on pressure from the fingers. Then apply Dr. Korinek's Absorbent Blister twice, 48 hours between applications. Repeat this treatment every two weeks until lameness subsides.

\section{Spavin-Bog}

CAUSE. Faulty conformation, slipping, falling through a bridge or culvert; large loosely built draft horses are prone to this blemish. Bog spavin is hereditary and you should therefore, select a good type of animal for breeding purposes.

SYMPTOMS. A puffy swelling located in front and on the inside of the hock, varying from the size of a walnut to that of a man's fist. It very seldom causes lameness, but is a serious disfigurement and blemish. 
TREATMENT. Ineffective unless taken in its first stage and when the animal is young. Apply Dr. Korinek's Absorbent Blister, rubbing in for twenty minutes at a time, every 48 hours for one week, then grease with common hog lard. Repeat this treatment in a month's time.

\section{Spavin-Bone}

CAUSE. Sprains of the hock from falling, slipping, jumping, pulling, traveling on uneven roads, falling through bridges, etc.

Since spavin is due to causes which come into-existence after birth, it cannot be regarded as an hereditary disease. Hereditary predisposition, however, is largely accountable for its appearance. In the first place, the process of evolution, in the horse, which is a single-toed animal, descended from a five-toed ancestor, predisposes him to suffer from union of the bones of the hock, just as it predisposes him to splints. The weaker the bones of the hock in comparison to the weight of the body, the more inclined will the animal naturally be to contract spavin.

SYMPTOMS. Spasmodic catching up of the spavined limb, the moment the heel of the foot touches the ground, something like the manner of string-halt. At times the stiffness can be observed only when the animal is pushed from one side of the stall to the other. Spavin may often be detected when riding a horse down a steep hill from the fact that he drags the toe:

The time of all others when a spavined horse will be apt to show his lameness will be the day following a hard day's work; and when he makes his first move from the stable in the morning is the proper moment for examination. Therefore, you should be prepared to form judgment quickly in these cases, for the longer the animal is trotted up and down, the less lame will he generally become.

We may have a visible sign of spavin, swelling and hardness of the part, without lameness. If there be heat and tenderness on pressure, lameness will almost always be present. A careful comparison should be made of the hocks.

TREATMENT. One important factor in treating spavin is keeping the animal quiet. This can be accomplished by placing the animal in a very narrow stall, carrying his feed and drinking water for a month or six weeks. Apply Dr. Korinek's Absorbent Blister twice with an interval of forty-eight hours, the first and third week of his standing.

To cure a spavin it is necessary to unite two or more bones of the hock, and I am sure that you will agree with me that a fractured bone cannot unite if moved frequently. The same thing exists in Bog Spavin only we have not the ragged edges of a fractured bone. 
The younger the animal, the easier the spavin is to treat, because bones harden with age and contain more mineral matter and less flexible animal matter. While treating the animal feed easily digestable foods.

\section{Stifle Joint Lameness (Dislocation of the Patella)}

Although this is a rare accident, it is the most common form of dislocation which occurs in the horse.

CAUSE. Young loose jointed horses are predisposed to dislocation of the stifle on account of the cornparative want of strength of their ligaments. They are much more liable to this accident than older horses, especially if they are in poor health or in rough, hilly pastures; the nature of which would naturally make them susceptible to the injury; however, it may take place as a result of accident at any age. Young horses that suffer off and on from dislocation, frequently lose this liability with increasing strength and age. The dislocation may be partial or complete. In the former case and the most common, the patella, or the little stifle bone that glides in the groove composed of the lower hip and upper thigh bones has become entirely dislocated or removed from its natural position.

SYMPTOMS. When the dislocation is complete the affected limb is drawn forward, while the foot from the pastern down is drawn backward, and the animal may throw weight on it when made to move, which is accomplished with great difficulty. When the dislocation is partial, the symptoms are about the same as mentioned, only the limb is less rigid. If the horse is moved the stifle makes a clicking sound. In this form the animal may have both limbs affected.

TREATMENT. Do not try to move the animal about while in this condition; the proper thing to do is to remove the cause as soon as possible. By placing an ordinary collar on the horse's neck and tying a rope to the under part, drawing it between the fore legs and passing it around the fetlock joint, have an assistant pull gently on the rope and when the leg is brought sufficiently forward by the assistant, the hand is passed over the region of the stifle bone and a hard, quick push inwardly will throw it back into place. Although this sounds simple it is sometimes accomplished with great difficulty. When the parts are in their natural position the horse is apparently well, walks about as if nothing had been ailing him. Animal should be placed in a stall where he cannot move forward or backward, having the rope attached to the collar and the foot, holding the foot forward and Dr. Korinek's Absorbent Blister applied to the part and rubbed in for at least twenty minutes. This will strengthen the ligaments and muscles and prevent further dislocation and trouble. After being in this position for twentyfour hours the animal is ready for work again. 


\section{Splints}

CAUSE. Splints are caused chiefly by trotting or running on hard ground. It is evident that the higher the action of the animal's legs and the heavier the horse, the greater the danger of his cuntracting this disease. Jumping is also a common cause of splints; especially in a horse unaccustomed to it, for with practice the animal learns to regulate his movements so as to diminish to some extent the disagreeable if not actually painful effects of concussion.

SYMPTOMS. A splint is detected by grasping the horse's leg with the fingers upon one side and the thumb upon the other, and tracing the inner and outer splint bones from their heads downward to their tapering extremities. Any actual enlargement will at once arrest the hand; any rising or irregularity will create suspicion and lead to closer examination. Horses, especially young ones which have lately been put to work, not infrequently develop splints before any swelling appears on the bone. For this reason in examining a case of obscure lameness, particularly if the animal is young, do not fail to look for the sign of splint lameness, namely; that the lameness is abnormally greater at a trot than at a walk, and that the animal usually fails to bend the knees freely and grows worse with exercise. The last-mentioned condition is also present with corns, but an examination of the foot will determine the question of their existence. In young horses splints are sometimes mistaken for coffin-joint lameness or navicular disease.

To avoid this error, it should be remembered that, when brought on by navicular disease, the action of the limb improves with exercise; also that horses of five years of age or less very rarely suffer from coffin-joint lameness.

Some horses, owing to unusual development of the inner splint bones of the fore-legs, may appear to have splints, although careful examination may prove both limbs to be free from any bony deposit. When deciding such a point, note if the two inner splint bones are of the same size. Any swelling perceptible in a limb recently splintlameness is usually attended by heat and pain on pressure.

TREATMENT. If there is heat present, foment with hot or cold water and apply Dr. Korinek's Absorbent Blister at intervals of fortyeight hours every two weeks until four applications have been made. During this treatment, use the horse for slow work on soft roads, etc. As a rule the splint will not disappear at once but will gradually go away.

\section{Surfeit (Nettle-Rash-Urtecaria)}

CAUSE. The usual cause of Surfeit is supposed to be due to a character of food consumed which upsets the animal's digestive organs and the skin being continuous with the mucous membranes lining the intestinal canal, a disturbance of the one structure is 
readily communicated to the other. Apparently owing to the extreme dry nature of the forage during the greater part of the year, horses in the United States frequently suffer from Surfeit.

SYMPTOMS. Surfeit is a term applied to an eruption of small, irregular lumps or boils which are more or less painful to the touch and which break out suddenly as a rule on the horse's body and neck, and, in rare cases, on the legs. A favorite seat of Surfeit is the parts covered with the harness or saddle and along the neck and withers. Surfeit is very troublesome and annoys both the horse and driver, especially when the horse perspires, as they rub violently when coming in contact with any object.

TREATMENT. Give two to four ounces of Epsom Salts in hot wheat bran mashes every morning. Feed as much sloppy food as possible, vegetables, etc. Avoid feeding dry, woody hay, as it irritates the intestines and aggravates the disease.

\section{Sweeney-Atrophy}

CAUSE. An ill-fitting collar, one tug longer than the other, striking an object when pulling - as a stone or the corner of a building - a splınt, spavin, ringbone, sidebone, coffin joint, lameness, curb, corns, stıfle lameness, or in fact anything, that tends to make an animal favor or abstain from using certain muscles. Sweeney is not a disease, but a retarding of the development of the muscles, which waste away or shrink when not used as nature intended Perhaps you have seen persons who had suffered a fracture of the leg or arm, and through not using the limb, the muscles would waste or atrophy and remain in this condition until they were again normally used.

SYMPTOMS. Locate the cause. The animal may be very lame, although sometimes any lameness is very difficult to detect, and where it is present, is usually due to ill-fitting collars. Remember, an animal may have a sweeney of the hip as well as of the shoulder, and keep in mind the above mentioned causes.

TREATMENT. When you have the cause of the sweeney located it is easily treated as a rule. Dr. Korinek's Absorbent Blister is effective in all cases except where the trouble is caused by corns, then use Dr. Korinek's Hoof Remedy. Bear in mind that it takes considerable time for the wasted muscles to regain their normal condition.

\section{Thoroughpin}

CAUSES. Generally due to some irritation of the hock joint, such as severe sprains from animal jumping, slipping, kicking or falling through a culvert or bridge, or it is frequently due to hereditary, so be very careful in choosing a sire when breeding.

SYMPTOMS. Sometimes there is lameness when the thoroughpin is first noticed, but it will gradually disappear as soon as the inflammation ceases. There will be a puffy soft enlargement, which 
occurs at the upper and back part of the hock, beneath the great tendons. Generally both sides are enlarged and puffy, but occasionally it happens that one side only is involved. Thoroughpin is also a fore-runner of Bog Spavin, as they are generally connected. The hock contains joint oil, as all other joints do, and is retained in place by a thin white fibrous membrane. Irritation of the hock joint tends to develop an extra large quantity of joint oil, and, as the hock is less protected by tendons when a thoroughpin or Bog Spavin occurs, those puffy swellings are filled with joint oil and are connected. If you press on one side of a thoroughpin you will see the other side bulge out. If you press on a Bog Spavin and there is a thoroughpin present, you will see it bulge on either side of the thoroughpin-or vice versa.

TREATMENT. If an old horse and of long standing, thoroughpin cannot be very satisfactorily treated, but if the animal is young treatment is often successful. Never attempt to open or lance these puffy swellings or you will produce an open joint. A good blister such as Dr. Korinek's Absorbent Blister is the only treatment that I could recommend. Make two applications, 48 hours apart, repeat treatment the following week. Remember to rub in well. There will be no danger of leaving a scar. Speedier results will be obtained if the horse be kept very quiet.

\section{Tapeworm (Taenia)}

These worms have been found in the horse, but so rarely that they need not be considered. When present they are more or less injurious to the animal.

\section{Thrush}

CAUSE. The two main causes of Thrush are lack of pressure on the frog and the decomposing effect of filth and fermentation of organic matter which accumulate in the cleft of the frog.

SYMPTOMS. The animal in some cases is lame, there will be a swelling accompanied by a very fetid discharge, in some cases the frog has practically rotted away, there will be more or less inflammation in the foot. The legs may even swell. Thrush is more frequently found in the hind feet because of the manure and filth with which they must come in contact.

TREATMENT. Cut away all loose pieces of horn, then apply a good hot flaxseed poultice and leave it on for twenty-four hours, after which wash well with soap and warm water. Then mix thoroughly together one teaspoonful of calomel and one tablespoonful Dr. Korinek's Hoof Remedy and apply to groove in the frog. Keep the foot clean and do not allow the animal to stand in filth. 


\section{Wind Gal's}

CAUSE. By sprains from slipping and concussion, and from fast work on hard roads.

SYMPTOMS. When concussion alone is responsible, the suspensory ligament and the back tendons will at first be in a normal condition, and the swelling will probably be confined to both the inside and outside of the leg, and may be felt in the form of a puffy swelling on each side of the fetlock by placing the fore-finger and thumb on the joint. In more serious cases resulting from sprains the vacant space between the back tendons and the suspensory ligament may also become filled with fluids. In other words, a wind gall has formed. In some cases the animal may be lame.

TREATMENT. When there is heat present apply cold, wet packs until the heat disappears, then apply Dr. Korinek's Absorbent Blister, rub in well for twenty minutes and repeat at intervals of 48 hours. This treatment is not always successful so do not be discouraged if the enlargements do not disappear.

\section{Wounds}

Wounds caused by external injuries have a general resemblance, and whether clean-cut, punctured, lacerated, poisonous, gunshot, etc., they require practically the same treatment.

TREATMENT. First stop excessive bleeding. There are several methods of accomplishing this, such as tying the artery with clean thread, searing with a hot iron, twisting the artery with forceps, or with compressed bandages. After the bleeding is suppressed and all foreign matter and ragged pieces of flesh removed from the wound, wash with Dr. Korinek's Dip and Disinfectant, one teaspoonful to a pint of water, or a carbolic acid solution of the same strength.

SEWING OF WOUNDS. I cannot say that I am in favor of sewing wounds unless they are gaping badly. After the wound is clean dust on Dr. Korinek's Antiseptic Gall Powder. Heavy cotton thread may be used for sewing the wound after being saturated in the antiseptic solution as above mentioned. Twitch the animal's nose or you may be compelled to throw him. 


\section{Wolf or Supernumerary Teeth}

Wolf teeth are comparatively small in size and have only one root and are found just in front of the upper molar teeth. Sometimes they do harm, but that is an exception and not the rule. They can be easily removed with a pair of small forceps or in some instances may be punched out.

I think they interfere with the eyes, causing them to become watery and inflamed. This is due to the tooth exerting some influence upon the optholmic division of the fifth nerve.

Supernumerary tooth substance may develop in almost any part of the body. This substance is called supernumerary teeth and is most commonly found in the testicles, ovaries or sinuses of the head.

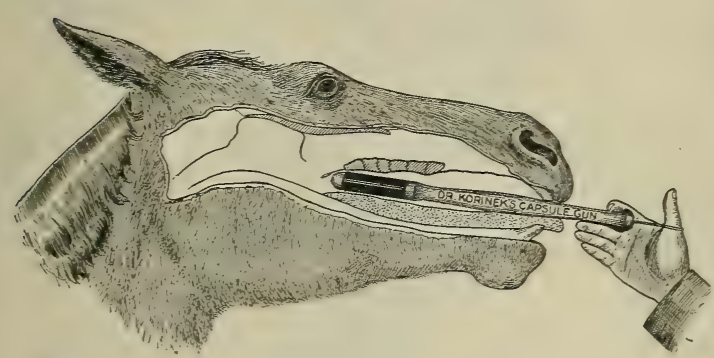

METHOD OF ADMINISTERING DR. KORINEK'S CAPSULE REMEDIES. 


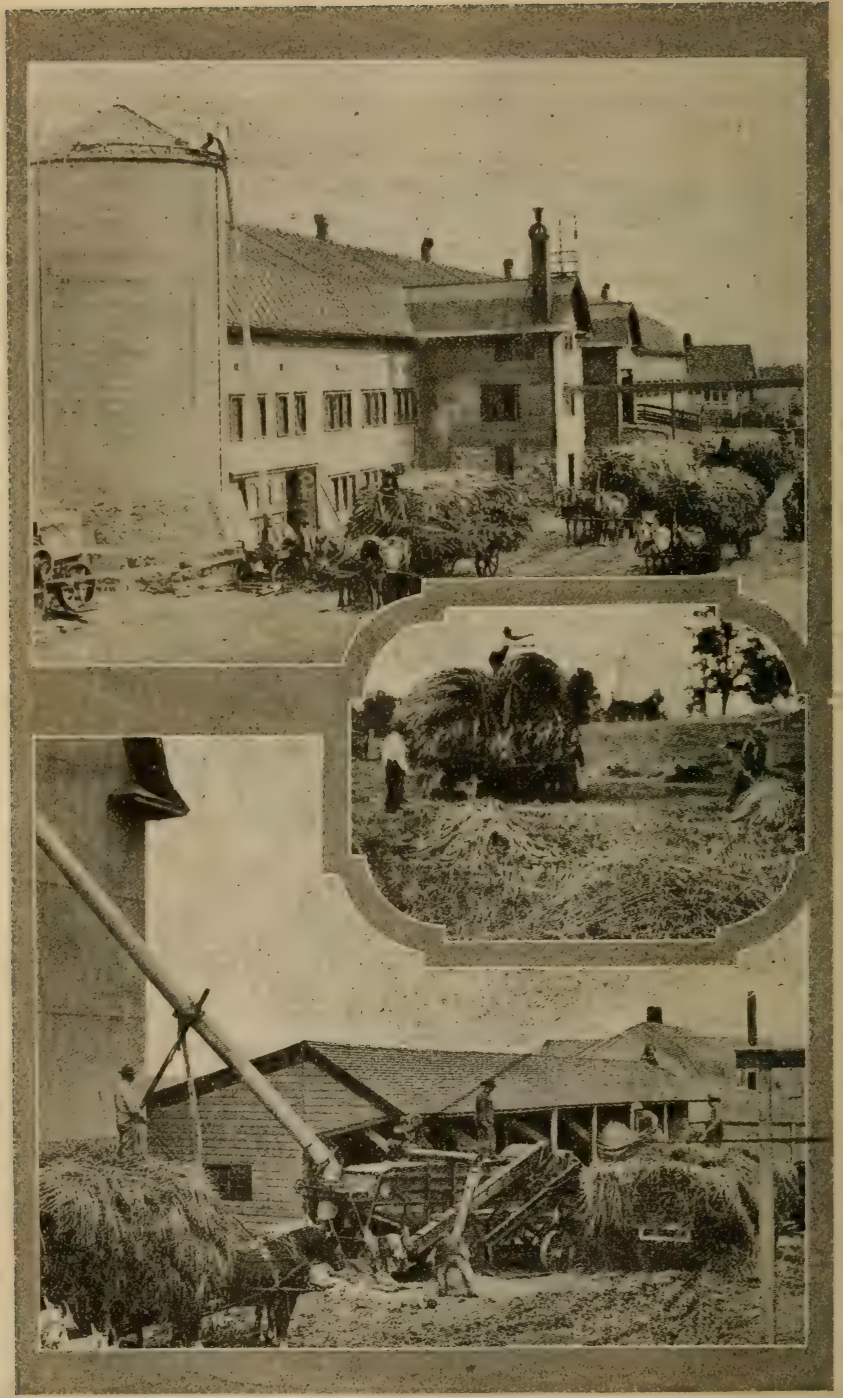

FILLING THE SILO 


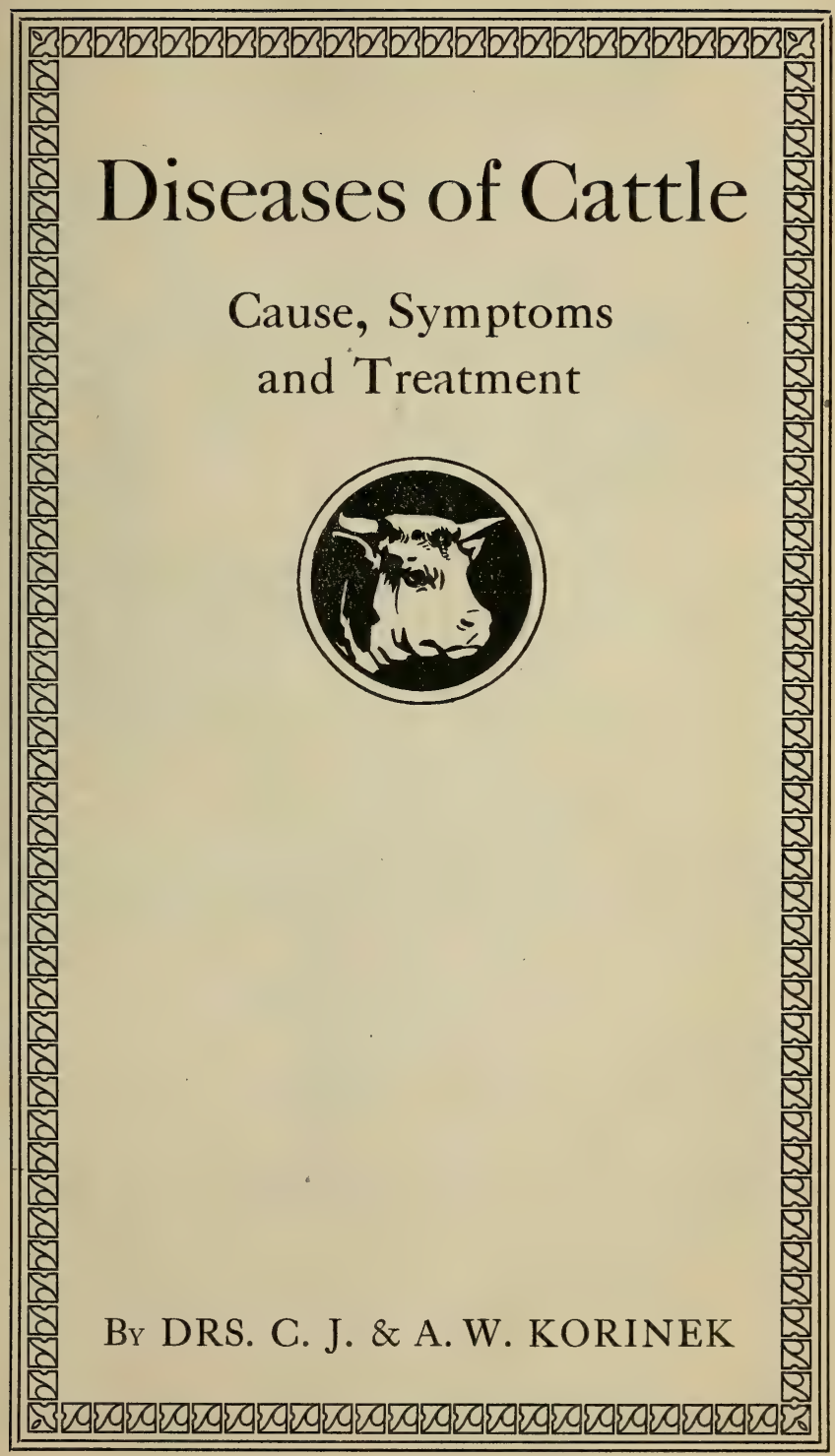




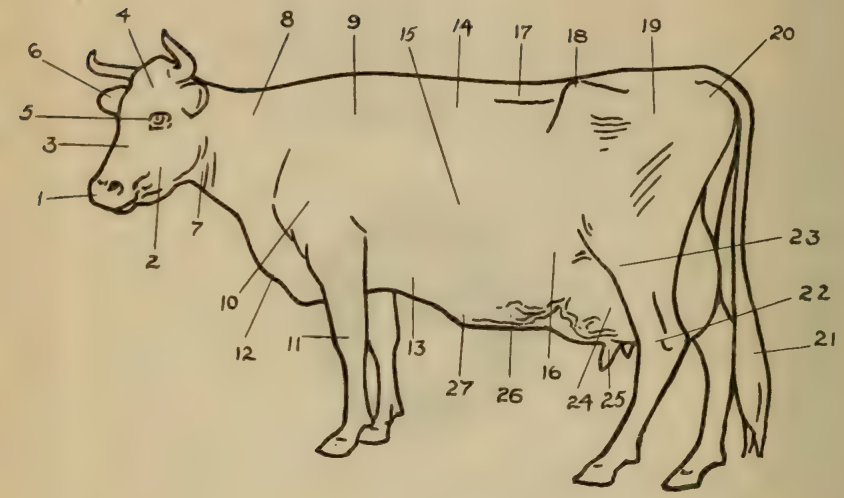

POINTS OF THE COW

$\begin{aligned} 1 & \text { Muzzle } \\ 2 & \text { Jaw } \\ 3 & \text { Face } \\ 4 & \text { Forehead } \\ 5 & \text { Eye } \\ 6 & \text { Ear } \\ 7 & \text { Throat } \\ 8 & \text { Neck } \\ 9 & \text { Withers } \\ 10 & \text { Shoulder } \\ 11 & \text { Knee } \\ 12 & \text { Dewlap } \\ 13 & \text { Chest } \\ 14 & \text { Back }\end{aligned}$

15 Ribs

16 Barrel

17 Loins

18 Hips

19 Rump

20 Pin Bones

21 Tail

22 Hock

23 Stifle

24 Udder

25 Teats

26 Milk Veins

27 Milk Wells 


\section{Abortion (Non-Contagious)}

CAUSE. Injuries from slipping or falling on icy roads, in box cars or deep gutters; receiving blows on the body, keeping the animal in unsanitary stables, eating poor food that may be irritating or poisonous, etc. In such cases the cow's vitality is in a run-down condition, so to speak, and the fetus dies and is expelled as a result. Losing large quantities of blood also produce abortion, or a cow heavy with calf placed in the same quarters with the cows that retain their after-birth also has a tendency to produce it. Intestinal worms, lung worms, liver flukes, cause an excessive drain upon the system or produce irritation of the digestive organs, consequently the cow gets very poor and emaciated. The above mentioned are perhaps the most common causes of "non-contagious abortion."

SYMPTOMS. The cow is uneasy, becomes separated from the herd, the bag and vigina may be slightly inflamed and perhaps discharging. If a cow heavy with calf and she craves eating dirt, rags, nails, etc., this indicates a lack of iron or phosphorus in her system and proper food should be provided for relief.

PREVENTATIVE TREATMENT. Provide clean, warm, comfortable quarters, good food, clean water to drink, do not subject to injuries, do not permit coming in contact with cows retaining their afterbirth.

The above is very important, especially if the cows are valuable and a large yield of milk is expected. If they have worms, treat the same as under special heading. If they have a loss of blood or a lack of mineral matter in their system, give Dr. Korinek's Kow Konditioner as directed.

\section{Anthrax}

Anthrax is a very contagious disease and can be communicated to all warm blooded animals and to man as well.

CAUSE. Due to the presence of a germ called the Bacillus of Anthrax and is one of the oldest diseases attributed to germs. These bacilla thrive in a warm climate, although sometimes found in cold countries. The infection is carried to various parts of the world by boxcars, ships, hides, hoofs, horns, wool and hair taken from sick or dead animals affected with Anthrax. This, perhaps, is the most common method of spreading the disease.

SYMPTOMS. Loss of appetite, grinding of the teeth indicating great pain, trembling of the muscles, temperature elevated to $104^{\circ}$ or $106^{\circ} \mathrm{F}$., breathing very rapid, pulse fast and weak, hair rough. 
There are some cases where animals are seized quickly with the disease and die very suddenly. This form resembles apoplexy. Carbuncles or abscesses are seen on the surface of the body in nearly all cases, a bloody discharge from the mouth and nose. The animal may paw the ground, rear in the air, run, and finally go into convulsions and die. This is termed "the furious form of Anthrax."

TREATMENT. Prevention is the most important. Fields or pastures that are infected with this disease should be burned every summer if possible, to destroy the germs. The animals that succumb to the disease should be buried deeply and quick lime thrown upon them; and blood stains upon the ground should have a strong disinfectant thrown upon them. The hide of such an animal should not be used, as the person removing it is likely to contract the same disease, especially if an abrasion is present on the hand, or such a hide or any portion thereof is likely to spread the infection after reaching the tannery, etc.

MEDICAL TREATMENT OR SERUM TREATMENT. This is the only thorough method of eradicating the disease, and when it once becomes prevalent in a locality the services of a competent veterinarian should be secured and all animals inoculated.

\section{Amaurosis}

Diminution, or complete loss of vision.

CAUSE. Paralysis of the optic nerve (nerve of sight) injury to the eye, or confinement in dark stables.

SYMPTOMS. Pupil fully dilated and glassy-sometimes called glassy eye. The cow carries the head high and steps high, which is very unusual in a cow. A blind cow would be a serious trouble to her owner.

TREATMENT. Nothing can be done for the cow after she is blind, but the condition may be prevented, and if detected in its early stages, the development of the disease may be arrested by the use of Dr. Korinek's Eye Remedy.

\section{Abscesses}

An abscess is a pus-containing cavity.

CAUSE. Injuries, bruises; tapping the cow when bloated, may result in an abscess if the operation is not properly performed.

SYMPTOMS. The condition is not always noticeable in the first stages of its development, but in time a well-defined swelling appears which is hard and tender to the touch. The hair falls from the affected part, which grows softer and less sensitive as it comes to a head. When ripe it breaks and discharges pus. 


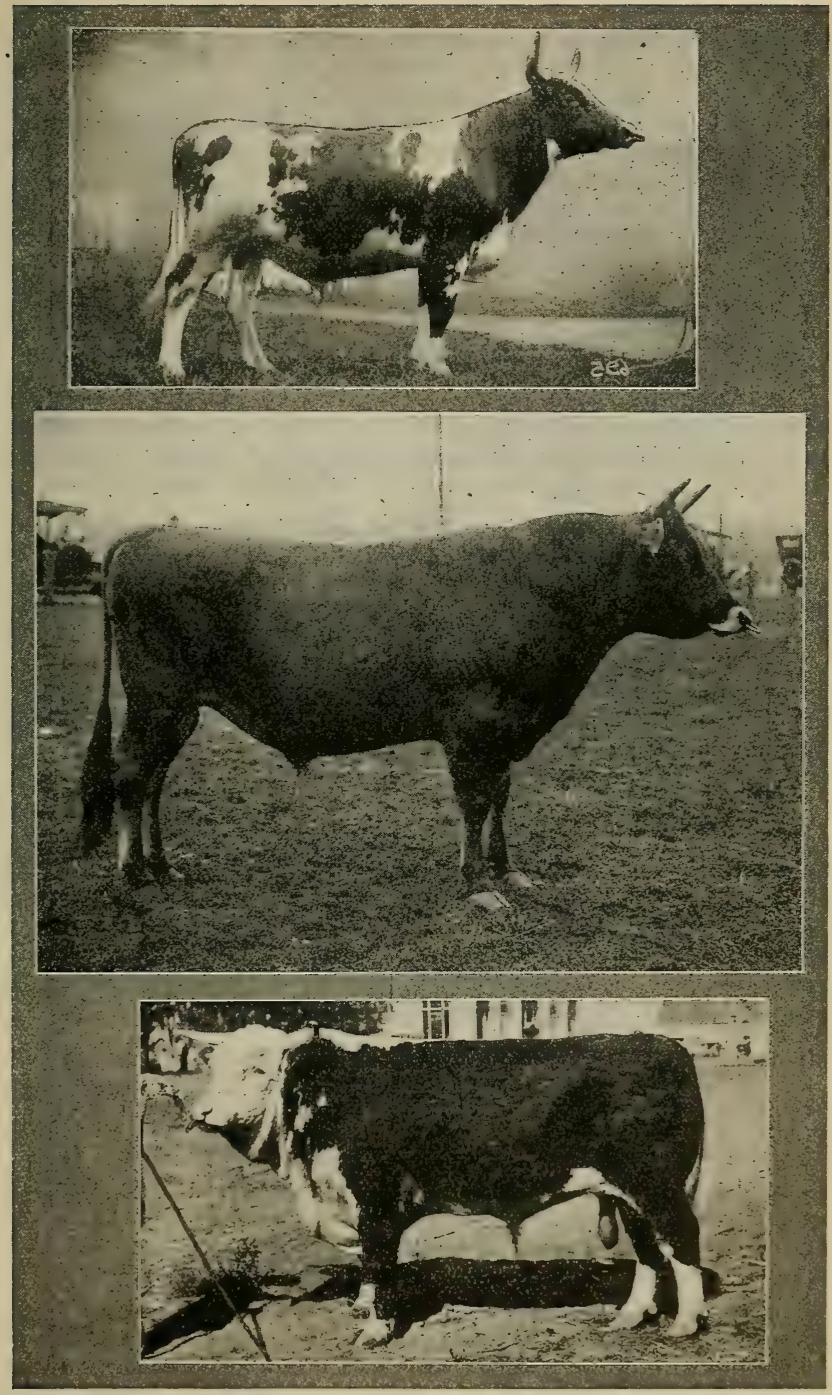

THOROUGHBRED SIRES 
TREATMENT. It is always better to allow the abscess to open naturally and without the aid of a knife, as there is danger of cutting an artery and causing the animal to bleed to death. As soon as the abscess breaks it should be drained and washed out thoroughly with Dr. Korinek's Dip, one teaspoonful to a pint of water. If it seems slow in coming to a head, apply Dr. Korinek's Absorbent Blister, as directed; this will hasten the ripening process. Give Dr. Korinek's Kow Konditioner, which is a great aid to the system in throwing off poisons.

\section{Blood Poison (Pyaemia; Septicaemia)}

CAUSE. Due to a septic infection taken into the blood, frequently found in cows with retained afterbirths, following intlam. mation of the womb or intestines, wounds and bruises of the skin and frequently found in calves affected with joint illness.

SYMPTOMS. High temperature, $103^{\circ}$ to $107^{\circ} \mathrm{F}$, pulse rapid and feeble, breathing increased, grinding of the teeth, the animal refuses to eat in most cases and ceases to chew the cud, although there may be great thirst present. Abscesses may form in various parts of the body, the membranes of the eyes and mouth will be congested with blood, giving them a dark-red appearance, although in the latter stages of blood poison this may change to a yellowish tinge. Constipation or diarrhoea may be connected with the disease. The animal dies from general emaciation from four to six days after the first symptoms are noticed.

TREATMENT. The treatment of this disease is very unsatisfactory and death is the usual result. Perhaps the best treatment used is Dr. Korinek's Kidney Capsules internally, which thins and purifies the blood, and if there are any abscesses or wounds steam with hot packs after which apply Dr. Korinek's Dip and Disinfectant. While in the process of recovery give Dr. Korinek's Kow Konditioner as directed and you will be well paid for the quick results you will get.

\section{Bronchitis}

Inflammation of the membranes lining the Bronchia.

CAUSE. Result of inhaling cold air when not accustomed to it. A bad cold, if neglected, may develop into Bronchitis. It may also be caused by drenching with irritating medicines if, as sometimes happens, the drench enters the air passages instead of the gullet.

SYMPTOMS. Loss of appetite; fever, temperature $104^{\circ}$ to $105^{\circ} \mathrm{F}$. by the fever thermometer; breathing short and labored; painful cough, indicating inflammation of the windpipe and bronchial tubes and if the ear is placed to the side of the chest, a crepitating or cracking sound may be heard. Discharge from the nose and mouth. 
- TREATMENT. Place the animal in clean, warm, comfortable quarters, well-ventilated but free from drafts; blanket well and give Dr. Korinek's Distemper Remedy, two tablespoonfuls on the tongue every three or four hours. If the fever is high, give Dr. Korinek's Fever Capsules.

\section{Bleeding (Haemorrhage)}

CAUSE. May be caused in various ways and is sometimes difficult to control.

TREATMENT. Dr. Korinek's Antiseptic Gall Powder applied to the wound will cause the artery or vein to contract, thus checking the flow of blood; bandages should then be applied and the animal kept quiet. If the location of the injury will admit artery or vein being tied with thread, it is well to do so.

Bleeding from the navel in the newly-born calf may be caused by the cow bursting the navel-string too near the body of the calf. After washing the umbilical thoroughly with Dr. Korinek's Dip (one teaspoonful to a pint of warm water) tie a strong string around the navel-cord and leave it there until it drops off.

Bleeding from the womb may be caused by pulling the afterbirth from the cow, or by laceration of the organ during calving. Wash out the womb with a solution of Zinc Sulphate, one teaspoonful to a pail of water. Keep the animal quiet and give plenty of cool water to drink.

\section{Blue Milk}

In this condition the milk becomes watery and of a bluish color.

CAUSE. Germ (Baccillus Cyanogenus).

SYMPTOMS. The only symptom is the bluish color of the milk.

TREATMENT. This condition yields readily to Dr. Korinek's Kow Konditioner and good rich food.

\section{Bloody Milk}

CAUSE. This condition may be caused by injury of the udder, rupture of an artery, etc., or it may be due to eating resinous plants.

TREATMENT. Secure one of Dr. Korinek's Milk Fever and Garget Apparatus, and treat the same as an ordinary case of garget. Great care should be exercised in milking as the condition may be aggravated. In addition to the above treatment give Dr. Korinek's Kow Konditioner. 


\section{Black Leg; Symptomatic Anthrax; Black Quarter}

CAUSE. Due to germs. The Bacillus of Black Leg gains entrance to the blood by wounds of the skin or the mucus membranes, lining of the mouth, and the intestines. This disease principally affects cattle, although sheep and goats may become affected with the same disease.

SYMPTOMS. This disease affects cattle that are in good condition and between the ages of six months and two years. In most cases death is very sudden, and perhaps the animal is found dead. The first symptoms are high temperature, from $104^{\circ}$ to $108^{\circ} \mathrm{F}$., los's of appetite, the animal stops chewing the cud, breathing considerably hurried, the joints of the limbs become swollen, also the chest and shoulders. All the swellings are painful on pressure and spread very rapidly over the body. The affected animals move with great difficulty and lie down frequently. If the hand is passed over the body, there is a peculiar crackling sound due to the gas developing under the skin. At the most distended portions of the swellings the skin becomes dry and the animal apparently has no sense of feeling. If the skin is punctured at this place, there will be a darkred, frothy discharge which has a very disagreeable odor. There will be a shivering of the muscles and the animal dies from convulsions.

TREATMENT. Remove non-affected animals to non-infected pastures, and confine affected animals to as small a territory as possible. The carcasses of the dead animals should be buried deep and covered with lime or burned, being very careful that all blood stains on the ground where the animals have been skinned are thoroughly disinfected. Inoculation is necessary, and is the best form of treatment in localities where Black Leg exists. Inoculate or vaccinate the calves when six months old or over, although after the animal reaches the age of two years or over they generally become immune to this disease.

\section{Barrenness}

CAUSE. Overfeeding, food too rich or too poor in quality, or an insufficient quantity of food. Diseased generative organs, Contagious Abortion, Leucorrhea, or diseased ovaries. Malformations, abnormal growths in the womb, closure of the neck of the womb, etc. Inbreeding, using the same herd bull on his own offspring. Breeding too young, offspring takes nourishment away from the young heifer, which is necessary to complete her maturity.

When a bull and a heifer are born twins, one or the other is unable to reproduce. Heifers become muscular, bag is undeveloped, and they resemble a steer.

Overstimulation of the generative organs, excessive secretions, or to inactivity and absence of secretions. 
TREATMENT. In some instances it is impossible for cows to breed, in which case it is best to market them. In other cases, a good breeding tonic is all that is necessary. Give Dr. Korinek's Kow Konditioner as directed on the can. If the neck of the womb is contracted, it should be dilated. If due to Contagious Abortion, the cow should be treated for the disease.

\section{Bloating}

A very common disorder in cattle and characterized by a puffedup appearance of the left flank. The cow has four stomachs, of which the rumen is the largest, its capacity being about fifty gallons in the average cow, and it is this stomach which fills with gas when a cow bloats.

CAUSE. Eating green clover or alfalfa; even when a cow is accustomed to this diet, it may cause bloating if wet with dew or rain; cured alfalfa (hay), moldy or frozen mangles will also produce bloating; the above-mentioned foods undergo a process of fermentation which causes excessive formation of gas, and death may result very quickly. Death may be due to rupture of the stomach or the diaphragm (muscle separating the abdominal and lung cavities), but is more often due to suffocation caused by distention of the stomach, which becomes so large that it pushes the diaphragm forward against the lungs in such manner as to stop their movement and the animal smothers. When the cow falls it indicates that one of these possibilities has occurred and death follows quickly.

SYMPTOMS. Cattle usually bloat suddenly and without warning; the left flank becomes abnormally swollen; back is arched, breathing labored; sometimes the tongue hangs out and the animal bellows. When rupture or suffocation occurs, the animal reels, staggers, and falls, after which nothing more can be done.

TREATMENT. Give Dr. Korinek's Anti Cow Bloat Capsules as directed upon the package; in extremely bad cases it is well to gag the animal by placing a piece of wood about two inches thick across the mouth like a bit and tying it in place with a rope passed over the crown of the head.

NOTE. We would emphasize the fact that since death from bloating occurs so quickly, this remedy should always be at hand and have a prominent place in every stockman's medicine case.

\section{Cough (Acute and Chronic)}

A cough is a symptom of various diseases, which in addition to the cough, must be treated.

KINDS OF COUGHS. Many writers give several different varieties, but for the sake of convenience, I divide them into two forms, namely, Acute and Chronic. 
CAUSE. Acute coughs are generally due to sudden exposure to cold, drafts; coughs are the forerunning symptoms of diseases of the organs of breathing.

Chronic coughs are associated and often a result of sore throat, lung fever, pleurisy, bronchitis, and catarrh.

TREATMENT. Give Dr. Korinek's Distemper Remedy as often as necessary, and do not expose the animal to sudden changes of temperature or inclement weather.

\section{Calving}

Signs of normal calving are, firmness and enlargement of the udder, extending well forward following the milk veins. The teats as a rule discharge a thin milky fluid, relaxation of the muscles on each side of the crop or the base of the tail. The outer surface of the womb becomes swollen and inflamed, discharging sticky, stringy, transparent mucous. The cow becomes uneasy, stops eating and if in a pasture becomes separated from the rest of the herd; will lie down and get up alternately as if in great agony. When birth pains start, the back is arched, and a severe straining follows by the contraction of the abdominal muscles. The membranes covering the fetus will be the first to make their appearance, engorged with a fluid from the womb. This is commonly known as the water bag, which eventually bursts and the two fore-feet can be seen, and, as the animal continues to strain, the nose and head will next be seen. When the calf's shoulders are exposed, the cow as a rule rises suddenly to her feet and the entire calf is expelled, also, the membranes surrounding it, but the membranes next to the womb as a general rule remains longer and sometimes requires artificial assistance to remove it. (See Retention of the Afterbirth.) Destroy the fetal membranes by burning or burying them deeply. Do not permit the cow to eat them. Wash the calf's navel with Dr. Korinek's Dip one part to 70 parts of water once or twice a day until the navel is thoroughly dry, as an infection may follow, producing joint ill or scours, from which death may result.

ABNORMAL CALVING. This is a condition where the fetus is not normally presented, as that just described. The feet may be presented in a normal manner, but the head and neck twisted back or to one side, or the head and one fore-foot may be presented normally, while the other fore-foot is doubled back, or there may be a breach presentation, as the rump of a fetus with both hind feet thrown close to its body. This is a very difficult presentation, especially if in a young animal. A fetus abnormally presented requires good judgment and cleanliness, also lubrication of the walls of the womb with unsalted lard, cotton or sweet oil. Endeavor to place the fetus in as near a normal position as possible before any force is used in its delivery, although where both hind feet are presented. 
it is advisable to remove it in this position. The hands and ropes should be clean and washed with Dr. Korinek's Dip and Disinfectant one to $\mathbf{5 0}$ of warm water. It is not only dangerous to the animal, but to the operator as well, if proper antiseptic precautions are not practiced.

Space does not permit me going into details on various fetal presentations nor does it permit me to explain the exact methods or operations I would use in each particular case. Nevertheless, good judgment and cleanliness are important. Do not hurry. Take your time, and you will be successful in most cases.

When the fetus is removed and the afterbirth does not come away within twelve or eighteen hours, remove it. (See Retention of Afterbirth, which will describe its means of attachment and its removal.)

\section{Cataract}

Destruction of the lens of the organ of vision, and seldom occurs in cows. The blindness may be partial or complete.

CAUSE. Opthalmia is the forerunner of this disorder, or it may be due to injury.

SYMPTOMS. If the pupil of one eye appears larger than the other, it is well to make a careful examination, as this is one of the first signs of a Cataract; this is followed by partial or complete blindness and white pearly deposit in the lens of the eye.

TREATMENT. Very little, if anything, can be done. Beneficial results are obtained by using Dr. Korinek's Eye Remedy as directed.

\section{Catarrh}

Not a very serious disorder in itself, but if neglected, may terminate in Lung Fever or Bronchitis.

CAUSE. Exposure to cold and wet weather; usually comes on in the spring or fall.

SYMPTOMS. Sneezing, coughing, dullness and at times loss of appetite; nostrils dry and very much reddened in the first stages, but after the fever breaks, a profuse discharge is observed.

TREATMENT. Place the cow in a dry, well-ventilated box stall, blanket well and give her plenty of bedding. Give Dr. Korinek's Distemper Remedy, one tablespoonful every three hours, or in extreme cases, every two hours; inhalations of steam from boilinghot water and turpentine are beneficial. Dr. Korinek's Kow Konditioner will aid recovery and quickly restore the normal supply of milk. 


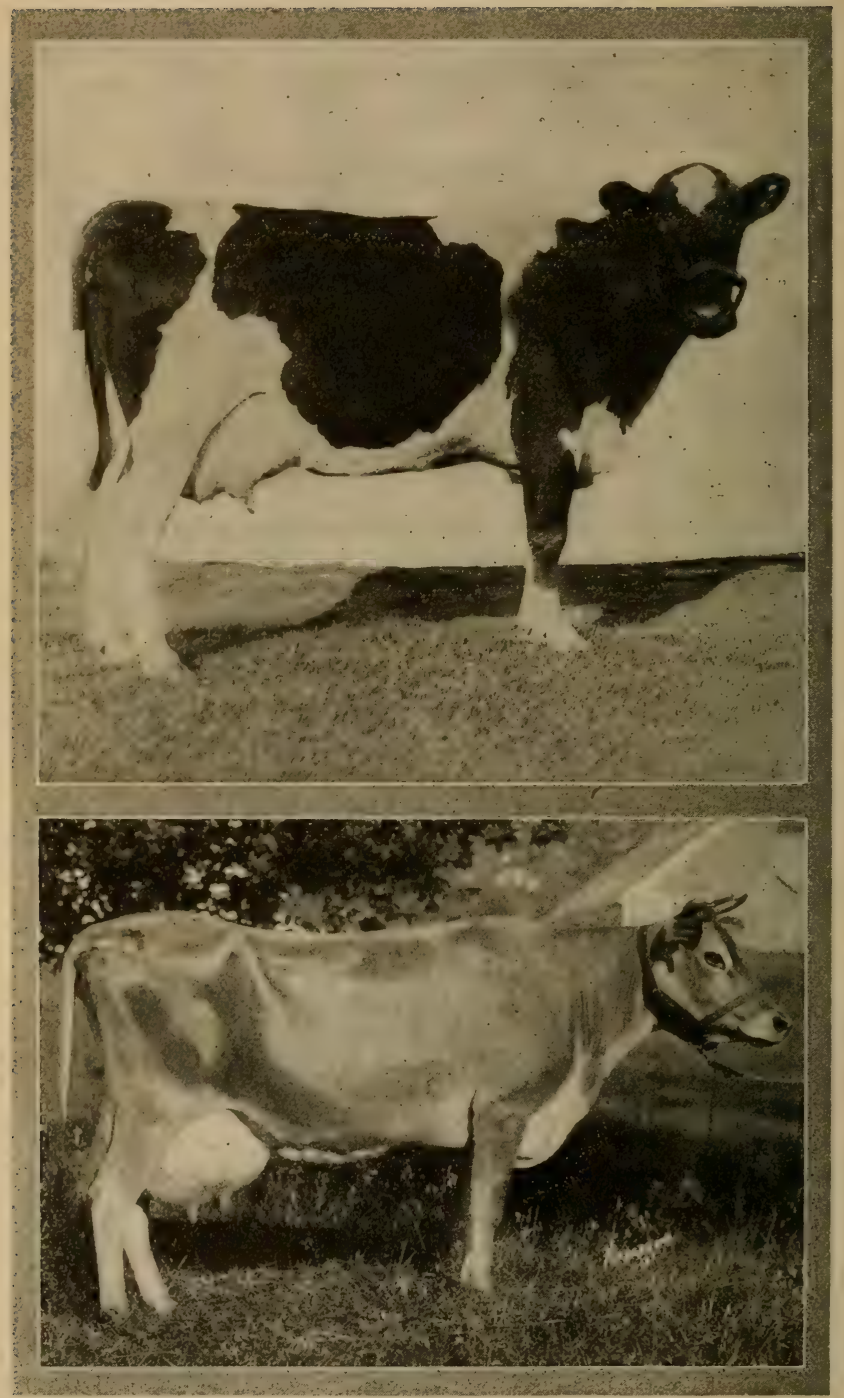

PRIZE WINNING MILCH COWS 


\section{Cow Pox}

A germ disease affecting the skin of the udder.

CAUSE. Disease is usually transmitted from one cow to another by the hands of the milker.

SYMPTOMS. Slight fever; udder and teats are red, swollen and tender. After three or four days, small pimples or pustules will appear about the size of a pea on the teats; these spots become larger and within a few days may be an inch in diameter. At the end of the second week the pimples burst, discharging an amber-colored fluid, and leaving raw sores, which cause the animal to suffer intensely when being milked. The supply of milk is also markedly decreased.

PREVENTION. The same person should not milk both the healthy and the diseased cows unless the hands are thoroughly washed in a carbolic acid solution and clothes changed that are likely to come in contact with the disease, as coat sleeves, etc.

TREATMENT. Give one of Dr. Korinek's Physic Capsules, and apply Zinc Oxide Ointment to the udder, as directed; this affords immediate relief and the sores heal rapidly. Dr. Korinek's Kow Konditioner will soon restore the health of the cow and the normal supply of milk.

\section{Calf Scours \\ (White Scours in Calves, or Calf Cholera)}

CAUSE. A specific germ, peculiar to the newly-born calf; often times associated with Navel-Ill, becoming infected through the umbilicus.

SYMPTOMS. Usually appears from the first to the third day after birth though it may come later; every symptom of diarrhoea; passages yellowish-white in color, foamy and very offensive to smell. Animal is dull and weak; back arched; hair dull looking; violent straining; discharge of mucous from the nose, muzzle is hot and dry. Calves lose flesh very rapidly and die in a short time if not given special care and treatment.

TREATMENT. Give Dr. Korinek's Calf Scour and Cholera Remedy as directed, and disinfect the barn to prevent the spread of the disease. Dr. Korinek's Dip, one tablespoonful to a gallon of water is excellent for this purpose.

Ordinary Diarrhoea, Bloody Flux, and Dysentery are also treated successfully with Dr. Korinek's Calf Scour and Cholera Remedy. 


\section{Choking}

CAUSE. A blocking of the oesophagus (gullet) caused by the animal attempting to swallow apples, potatoes, roots, dry grain, etc.

SYMPTOMS. Stringy discharge of saliva from the mouth; violent coughing, wrenching of the head and neck. The animal will bloat very quickly if the passage is completely obstructed, because it is through this channel that, in health, gasses escape from the stomach of a cow.

TREATMENT. Locate the obstruction as quickly as possible and if within reach, insert the hand and extract the object. Do not attempt, except as a last resort, to push the object down into the stomach, as there is great danger of rupturing the gullet. Try rather by gentle manipulation on the outside to push the object upward; if this fail, call in a good veterinarian, as experiments are likely to prove expensive to the owner.

\section{Colic}

CAUSE. This disorder is uncommon in cattle that are not worked. Oxen were much subject to it through drinking unlimited quantities of water when in an overheated condition.

SYMPTOMS. Kicking or raising the feet to the belly; alternate lying down and getting up; distention of the abdomen, stomach or paunch with gasses, chills, etc.

TREATMENT. Give Dr. Korinek's Colic Capsule or Dr. Korinek's Anti-Cow-Bloat Capsules, as directed.

\section{Coal Oil Poisoning}

Coil-oil is an old-fashioned remedy that is only used in the absence of something better. It is unsatisfactory and even darigerous, as an over-dose often results in poisoning.

SYMPTOMS. Owing to the irritating effect upon the stomach, there is an abnormal flow of stringy saliva from the mouth, looseness of the bowels, loss of appetite, watery discharge from the nose and eyes and sometimes convulsions and death, if a large enough dose has been given.

TREATMENT. Give one of Dr. Korinek's Colic Capsules morning, noon and night; feed easily-digested food and allow plenty of cold water.

\section{Casting the Withers (Eversion of the Womb)}

CAUSE. Weakness or relaxation of the organ and failure to contract after calving. The womb should contract immediately after the placenta (afterbirth) comes awav. 
SYMPTOMS. A large red-colored mass protrudes from the vulva with numerous small mushroom-like bodies (Cotyledons). Animal may lie or stand; when lying, there is danger of the womb becoming lacerated or covered with dirt.

TREATMENT. This condition is best avoided by feeding Dr. Korinek's Kow Konditioner during pregnancy, which will invigorate the generative organs and insure normal calving.

Great care is necessary in returning the womb to place. First wash the entire surface with a solution of Dr. Korinek's Dip (one tablespoonful to a gallon of warm water) and place the cow in such a position that the head will be lower than the hind quarters; then, supporting the womb with one hand, and manipulating with the other, gradually push the organ back into place. Do not try to force it all back at one time; the work should be done slowly and well and may take some time. Having replaced it, insert the hand to make sure it is in proper position. If the cow continues to strain and attempts to force it out, keep her standing with her hind quarters elevated and wash out the uterus once daily with Dr. Korinek's Dip (same proportion as above). Feed Dr. Korinek's Kow Konditioner; it will put tone into the generative organs and restore the full supply of milk in a very short time.

\section{Chronic Diarrhoea (Chronic Bacterial Dysenteria)}

This disease is peculiar to cattle and is characterized by diarrhoea, rapid loss of flesh, and is incurable.

CAUSE. A bacterium which invades the small intestines; may be picked up in the drinking water or while grazing on infected pastures.

SYMPTOMS. Diarrhoea, appetite is normal but the movements of the bowels varies according to the nature of the food eaten; rapid loss of flesh regardless of the quality of food.

PREVENTION. Destroy diseased animals and disinfect barn, barn-yards, etc.; do not allow healthy cattle to graze on infected pastures.

TREATMENT. Thus far no treatment has proven effective.

\section{Chapped Teats}

CAUSE. Anything that tends to irritate them. A sudden chilling of the teat in cold weather, after the calf has just let it go or after the operation of milking with wet hands or from an animal wading through deep water or tall wet grass. Also filth or irritants coming in contact with teats when lying down.

TREATMENT. Remove the cause if possible and dry the teats well after each milking and apply Zinc Oxide Ointment. Feed laxative food that is easily digested, as it has a very good affect on the blood, consequently it promotes the healing of wounds. 


\section{Diarrhoea}

CAUSE. An abnormally frequent and watery movement of the bowels due to overdosing with physics, irritants, poor food; intestinal worms, indigestion, etc.

SYMPTOMS. Frequent discharge of a thin and watery nature from the bowels, the movements are attended with great pain and sometimes streaked with blood; loss of appetite not unusual.

TREATMENT. If the disorder is caused by indigestion, poor food or poisons, give Dr. Korinek's Physic Capsule, but if due to a severe physic give Dr. Korinek's Diarrhoea Capsules as directed on package; followed in either case with Dr. Korinek's Kow Konditioner, which will tone up the digestive organs. When treating for Diarrhoea, do not allow too much water at any time. For calves use Dr. Korinek's Calf Scour and Cholera Remedy.

\section{Garget}

A congested condition of the udder, common in heavy milkers.

CAUSE. Not always easy to determine; may be due to injury of the udder, or lying on cold, damp ground, etc., in heavy milk cows before or following calving.

SYMPTOMS. Some parts of the udder may be swollen; milk is thin and watery and may be tinged with blood, and later becomes charged with small clots or cheese-like particles which are forced out during the milking process.

TREATMENT. Give one of Dr. Korinek's Physic Capsules, and use Dr. Korinek's Milk Fever and Garget Apparatus as directed. Give two tablespoonfuls of Dr. Korinek's Kow Konditioner three times daily, and feed soft, sloppy food.

\section{Dislocation of the Eyeball}

This rarely occurs in cattle. It may, however, follow a blow or kick from a horse. The muscle holding the eyeball in place is ruptured and the eyeball forced from its socket.

TREATMENT. If the injury has not been neglected, and the eye is still warm, it may be replaced, cutting the lid at the outer corner if necessary, to return the eyeball to place, and taking a stitch where the incision has been made. The eye should then be bandaged to hold it in position. If it should be necessary to remove the eyeball, a veterinarian should be employed to perform the operation. 


\section{Dehorning}

Dehorning is not considered a cruelty, for horns on cattle do not add to their comfort and lessens the owner's risk. It is much better, safer and, I think, more humane to prevent the growth of horns on calves after they are three or four days old by rubbing the elevations where the horns make their appearance with a pencil of caustic potash after being moistened with cold water. Repeat this application two or three times, which is generally sufficient.

When dehorning cattle with clippers or saw, it is well to remove about one-half inch of the flesh of the horn. This gives their head a much better appearance after the horns are removed and healed. When a horn is freshly amputated, apply oil of tar occasionally, as it is an antiseptic and prevents infection and the annoyance of flies. However, this should be performed during the season when the flies are less numerous.

\section{Drenching}

This consists of pouring a dose of liquid medicine down the throat of an animal.

Drenching would be bad enough if the animal were well, but it is extremely dangerous when an animal is sick and in pain. To prevent the medicine from escaping from the mouth, the head is raised high in the air, in which position, and with the mouth open, it is almost impossible for the animal to swallow, and the medicine is apt to enter the windpipe and lungs, resulting in lung fever; drenching, in fact, is as bad and perhaps more dangerous than the disease.

\section{Capsulize Your Cattle}

Dr. Korinek's Capsules are easily given and cause no bad aftereffects. The animal gets the entire dose and without unnecessary irritation.

\section{Dropsy} where.

A collection of watery fluid in the abdominal cavity or else-

CAUSE. Usually due to old age or poor food. In calves it is sometimes due to the presence of worms which, through stimulation, may cause excessive secretion of digestive fluid (chyle), resulting in Dropsy.

SYMPTOMS. Abdomen becomes abnormally large, especially underneath; the flanks sink in. The animal has poor appetite and walks with a weak, staggering gait; the mucous membrane of the mouth, nose and eyes is pale and bloodless.

TREATMENT. The cause should first be determined and the disease treated accordingly. If due to worms, give one of Dr. Korinek's Worm Capsules. If caused by lack of nourishment, improve the quality of the food, give bran mash containing a cup of oil-meal daily, and Dr. Korinek's Kow Konditioner in large doses. 


\section{Eczema}

A non-contagious disease of the skin common to caltle and characterized by red and inflamed condition of the skin.

CAUSE. Due to feeding on decayed vegetation and lying on wet and unsanitary bedding, or by application of irritating drugs to the skin.

SYMPTOMS. Redness and scratching of the affected part, which is usually confined to a small area. Inflammatory stage is followed by eruptions and a glutinous discharge, terminating in scabs and a thickening of the skin.

TREATMENT. Determine the cause and remove. If due to poor food, improve the quality; feed laxative food, and a hot bran mash occasionally; if the bowels seem inactive, give one of Dr. Korinek's Physic Capsules. Allay itching by applying Zinc Oxide Ointment, and feed Dr. Korinek's Kow Konditioner, which will purify the blood and restore normal tone.

\section{Ergot Poison (Ergotism)}

CAUSE. Ergotism is produced by cattle eating fungoid growths which attack kernels and seeds of rye and blue grass, etc. These kernels or seeds grow dark in color and become abnormally large and curved in shape. The infected grass or hay when eaten by cattle contract the arteries, especially those of the legs, just above the feet, although all the arteries in the body are contracted to a certain extent. This disease is frequently seen in spring and summer.

SYMPTOMS. Ergot is prescribed in cases of bleeding, because of its contracting effect upon the arteries (closing or stopping the flow of blood) where the blood supply is the weakest, as in the extremeties. It is cut off and this, of course, causes the skin just above the hoofs to break or crack as though it were cut with a knife. This shuts off the entire supply of blood to the foot, which mumifies and the lower portion becomes gangrenous and eventually sluffs off. One of the first affects of ergot poison in pregnant cattle is abortion, due to the blood supply to the wound being shut off by its contracting effect on the arteries. Cattle are particularly susceptible to ergotism.

TREATMENT. When ergotism is so advanced as to produce sluffing of the feet, it is best to destroy the animal. If other animals are affected slightly, find out the cause and remove it. Look to the hay or pasture as the producer. Administer one-half ounce of Chloral Hydrate two or three times a day in their drinking water or mix it with sufficient quantity of flaxseed meal to fill an ounce gelatin capsule and give with capsule gun. If the skin is slightly broken above the foot, wash with five per cent. solution of Carbolic Acid. Where the feet have become gangrenous, amputation of the foot or feet is necessary, which is not advisable unless the animal is very valuable. 


\section{Feeds and Feeding}

Food contains water, ash, protein, crude fiber, carbohydrates, and ether extract or fat. These must be given in correct proportions in order to get the desired food value. If, for instance, the food allowance contain too much crude fiber, such as straw, stalks, etc., indigestion will result; if the ration contains an undue proportion of concentrated food, the bowels become sluggish and fail to perform their duties and in either case the result will be anything but profitable.

A cow should have at least 25 pounds of dry matter or ruffage every day and to get the best results, the concentrated food should be given between feeds of the dry matter. In addition to this the cow must have plenty of pure water at all times. If it is necessary at any time to change the feed, it should be done gradually to avoid creating digestive disorders, founder, etc.

The digestive organs of the pregnant cow are inclined to be sluggish, and her food should be of a laxative nature to keep the bowels open. Dr. Korinek's Kow Konditioner is an excellent regulator and fed in small quantities will keep the cow in good condition at little cost.

\section{Founder (Laminitis)}

CAUSE. Inflammation of the internal or sensitive structure of the foot due to overfeeding, overheating, standing on concrete or stony floors without exercise, and sometimes due to injury to the feet.

SYMPTOMS. Feet hot and tender; fever, temperature 104-105 F.; breathing very rapid; appetite remains good. Animal lies down most of the time and if made to walk, does so with great difficulty. One foot or all four feet may be affected. Founder in a cow reduces the secretion of milk.

TREATMENT. Give two of Dr. Korinek's Physic Capsules immediately followed by one of Dr. Korinek's Kidney Capsules morning, noon and night. Stand the animal in a running stream of cold water if possible, or put cold packs to the feet to relieve congestion and reduce inflammation of the parts. Feed Dr. Korinek's Kow Konditioner to restore the normal flow of milk and perfect digestion.

\section{Fluke in the Liver and Lungs}

CAUSE. This disease is contracted by cattle grazing on marshy lands. There are two different species of fluke that affect the liver and lungs of cattle. They are both flat, leaf-like worms. The common liver fluke is about one-half inch long, while the so-called American fluke is somewhat larger. In their life history these flukes depend on snails as intermediate hosts. At a certain stage of development the young flukes live on snails. They become encysted on stalks and blades of grass which are finally swallowed by grazing cattle. This disease is most frequently seen in young cattle. 
SYMPTOMS. The animal shows no marked signs at first, but eventually the appetite diminishes, rumination or chewing of the cud becomes irregular, the animal becomes dull, hide-bound, hair standing or staring, the visible mucous membranes of the mouth and eyes become pale and bloodless, the eyes discharge watery fluids, oozing down the face, temperature varying from two to three degrees above normal and milk supply, if in aged cattle, remarkably reduced. In all cases there is great thirst and the animal becomes very much emaciated and refuses to eat. Swellings about the belly and breast, etc. Diarrhoea at first, alternating with constipation, but finally becomes continuous. The diseased animal succumbs to the malady in from two to six months.

TREATMENT. Medical treatment is unsatisfactory. The disease will be prevented to a considerable extent by giving animals plenty of salt and in the marshes containing pools of water introduce or plant carp, frogs and toads into the infected tracts. These animals destroy the young parasites and feed upon the snails, which serve as their intermediate hosts. Or, prevent the cattle from grazing upon swampy marshes by fencing them out.

\section{How to Care for the Calf}

One of the most imporiant factors in raising good, strong, healthy cattle, is caring for the calves when young. In days gone by the average stockman was satisfied if he managed to keep the calves alive until green grass came. This lack of feed and care resulted in a stunted frame and weak digestive system which brought little profit to the owner. That the calves well repay the little extra cost and care is evidenced by the splendid condition of the dairy cows and the large quantity of milk yielded and the large frame which in beef cattle allows the animal to put on flesh and weight.

\section{Hollow Horn (So-called)}

The horns of cattle are normally hollow, although the core extends well into the horn. This, however, is merely a prolongation of the frontal bone which affords a point of attachment for the horn. A case once came to the notice of the writer in which a so-called "horse doctor" when called to attend a sick cow, diagnosed the disease as "Hollow Horn," and drilled several holes into the horns; unfortunately the gimlet broke, a piece of it falling into the frontal sinuse (a cavity of the head containing air), causing inflammation and death. The cow therefore died from the treatment and not from the disease. 


\section{How to Give Dr. Korinek's Capsules}

Insert the thumb and forefinger of the left hand into the nostrils of the animal, holding the head firmly; then with the Capsule Gun in the right hand and grasping it with firmness, insert it in the side of the mouth; when the mouth is well open, push the Capsule Gun far back on the tongue and press the little plunger and the animal will swallow the capsule. With the aid of Dr. Korinek's Capsule Gun, one man can usually induce the most vicious animal to swallow medicines. It may be necessary in some cases to place the animal in stanchions. By all means avoid drenching.

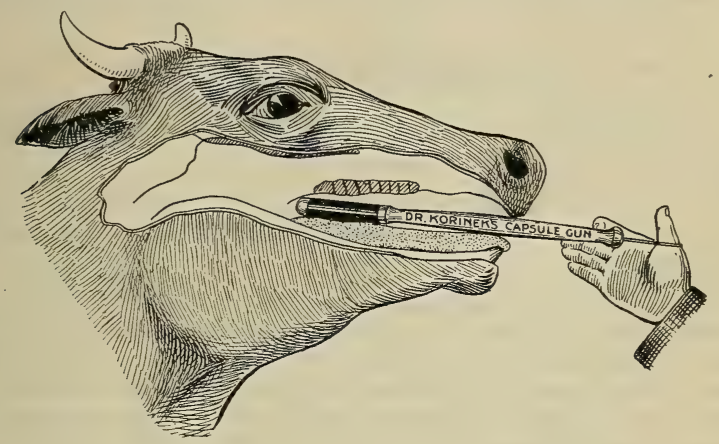

\section{Hard Milkers}

CAUSE. Due to contraction of the little sphincter muscle at the opening of the milk duct. Many an otherwise valuable animal is disposed of because of this defect.

TREATMENT. Write to the Korinek Remedy Company for method of treatment.

\section{Inflammation of the Eye (Conjunctivitis)}

Inflammation of the membrane of the eyeball and the lid.

CAUSE. Injury, weakness, irritating gases, foreign bodies, such as chaff in the eye, standing in dark and poorly-ventilated stables.

SYMPTOMS. A constant flow of tears runs from the eye which is inflamed and partly or completely closed. The lining membranes are very red (blood-shot).

TREATMENT. If due to foreign bodies, remove at once; in order to accomplish this the animal must be placed in stanchions, the neck twisted, and the eyelid turned back. Do not throw burnt alum in the eye; this will only make the condition worse. Apply Dr. Korinek's Eye Remedy as directed. 


\section{Inflammation of the Kidneys}

CALSE. Injuries in the region of the kidneys, sometimes caused by cows riding each other. Also due to irritating medicines, poisonous plants, drinking stagnant water, etc.

SYMPTOMS. Animal urinates often and in small quantities; hind legs spread out; breathing hurried, and in severe cases the temperature may be two or three degrees above normal. There may be swelling over the loins and as the disease progresses, the animal grows weaker and finally dies. Fortunately this disease is not common among oxen.

\section{Inflammation of the Penis}

CAUSE. Injuries received from snags, walking through the underbrush, jumping fences, etc.

SYMPTOMS. Arched back, swelling of the sheath and in some cases a discharge. It may be serious enough to affect the appetite and cause fever.

TREATMENT. Wash out the sheath three times daily with a solution of Dr. Korinek's Dip, one tablespoonful to a gallon of warm water (an instrument may be made for this purpose with a piece of hose and a funnel). After injecting, hold the opening tightly for a few minutes before allowing the liquid to escape. Repeat this treatment two or three times daily. Feed Dr. Korinek's Kow Konditioner and laxative food.

\section{Inflammation of the Womb (Metritis)}

CALSE. Inflammation of the lining of the womb is usually due to difficult labor, where the hands and instruments have been used; may result from a bruise received during the operation or from unclean hands or instruments - the veterinarian may cause the trouble if his instruments are not sterilized. Retention of the afterbirth if neglected may also cause inflammation of the womb.

SYMPTOMS. Signs of fever three or four days after calving; chills, arched back, stiffness of the hind parts, legs, ears and horns cold, nose dry and hot, grinding of the teeth indicating great pain, temperature two or three degrees above normal $\left(104-105^{\circ} \mathrm{F}\right.$. by an ordinary clinical thermometer). The cow stands still, ceases to chew her cud, and the secretion of milk is below normal. In a very short time a discharge from the womb is observed, watery and colorless at first, but which soon becomes thick and yellow and tinged with blood, showing that the lining of the womb is sluffing and being passed out; this discharge has a very offensive odor. The cow's condition is serious, requiring the best of care or she may die. 
TREATMENT. Give two of Dr. Korinek's Physic Capsules as soon as possible and flush the womb thoroughly with a solution of Dr. Korinek's Dip, one tablespoonful in a gallon of warm water (refer to cut showing how to give a uterine injection) three times daily for three or four days, after which twice a day is sufficient; continue until the discharge disappears. Dr. Korinek's Kow Konditioner should be given in doses of two tablespoonfuls three times daily; this will restore the milk supply quickly and put her in fine condition.

\section{Inflammation of the Heart Sack (Pericarditis)}

- CAUSE. Cattle being ruminants, therefore, not masticating their food finely before swallowing, consequently foreign bodies, such as nails, wire, etc., are picked up with the food and taken into the rumen or paunch. These sharp objects penetrate the walls of the paunch, rumen or first stomach and diaphragm the membrane or sack surrounding the heart, which produces an inflammation of the heart sack, or pericardium.

SYMPTOMS. Symptoms develop very slowly or indications of indigestion will be present as the appetite is variable, temperature elevated, breath labored, the animal avoids walking down hill as it causes them pain from the stomach and intestines pressing the lungs against the heart. The symptoms, however, are so slight that they may easily escape the notice of a casual observer. The animal eventually becomes poor, emaciated and dies. If you open the heart sack it will be found full of watery pus.

TREATMENT. Unsuccessful as this disease is seldom diagnosed correctly and if it was, an operation would be necessary, and this does not prove successful in the majority of cases.

\section{Inflammation of the Bag (Mammitis)}

CAUSE. Lying on the cold ground or concrete floors which chill the bag; injury to the bag by a kick or blow; also quite common in heavy milkers a day or two after calving.

SYMPTOMS. Chills, fever; ears, horns and legs are cold; bag is much enlarged and hard, red, and very tender to the touch; milk watery and nearly colorless at first, but later becomes tinged with blood and pus and has a fetid odor. This condition may affect all, or only one-quarter of the udder.

TREATMENT. Give two of Dr. Korinek's Physic Capsules; feed soft, easily-digested food and give plenty of cold water to drink. Purchase Dr. Korinek's Milk Fever and Garget Apparatus and follow directions closely; also apply Zinc Oxide Ointment to udder. Give Dr. Korinek's Kow Konditioner as recommended. 


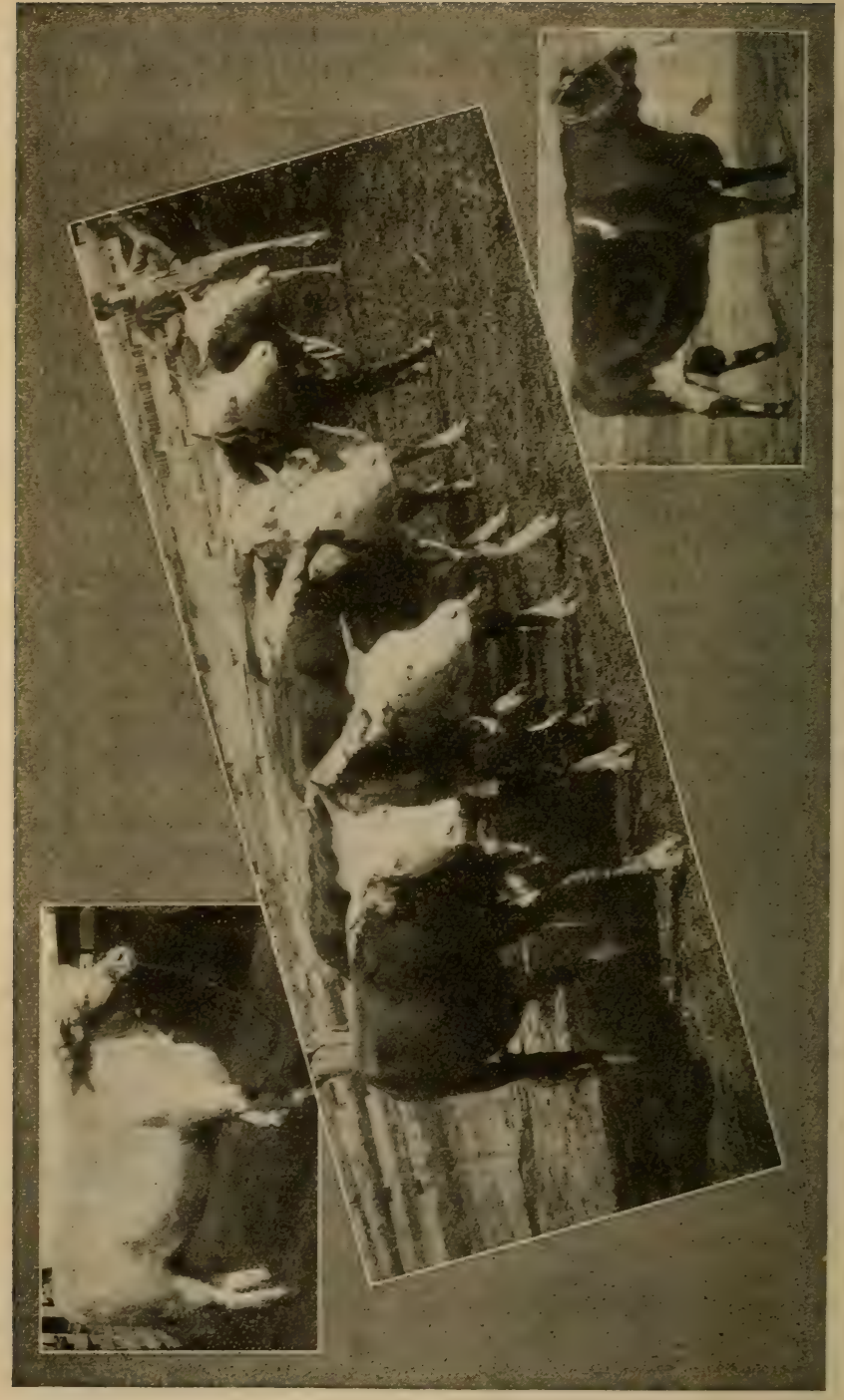

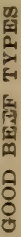




\section{Infectious Abortion (Contagious Abortion)}

This is a specific infectious disease caused by the Bacillus Abortus resulting in expulsion of fetus (premature calf). Infectious Abortion ranks second to Tuberculosis, from an economic standpoint, and in some localities supercedes Tuberculosis in its ravages. Not only the result of losing the calves, but a great shrinkage in the production of milk and failure to breed for several months and sometimes forever. It is common in both range and dairy cattle; the loss yearly reaches millions and is almost inestimable.

INFECTION. Infection or the introduction of Bacillus Abortus may be caused in various ways, but is usually introduced during copulation with the bull or importation of new stock into the herd. Although the infection may gain entrance through the digestive organs, or by rubbing the external genitals on rubbing posts or objects harboring the germs, but the usual means by which the disease spreads is by the herd bull.

SYMPTOMS. Period of incubation varies and is too irregular to base on a given time, some cases being more chronic than others or the animal affected withstands a more severe test or resistance against the invading germs. Two or three days before expulsion of fetus we notice signs of making bag. External genitals become reddened and swollen and sometimes small nodules imbed themselves upon the surface of the vulva, indicating great inflammation. A mucoid discharge is quite noticeable; sometimes it may have the appearance of pus, but as a rule it is odorless. The disease is much plainer in heifers than in older cows. Heifers will be seen making bag about the fifth or seventh month, in an older cow, of course, symptoms of calving are not so well marked.

Cattle affected with infectious abortion usually carry their calves from five to seven months, although it may occur in earlier months of gestation and the fetus and membranes are expelled unnoticed, which causes no systematic disturbance of a cow, but if the fetus is carried until later months of gestation, the afterbirth is usually retained or does not come away with the fetus. The fetus is usually dead and if born alive is puny and dies soon.

After abortion has occurred, a dirty yellowish discharge containing pus persists for several days and if the afterbirth is not removed in due time may result in inflammation of the womb and blood poisoning. After a cow has once had infectious abortion, she may be served by the bull several times before she will conceive and in some instances may never breed.

PREVENTION. Where the disease exists, change the uninfected cattle to new sanitary quarters or premises, keeping the diseased cows by themselves, or in a separate stable from the healthy ones. Each herd should have its own attendant, or one person should not take care of both the diseased and healthy stock. When any of the cows develop symptoms of abortion they should be treated with 
suspicion and removed from the healthy cows at once. After abortion has occurred, the fetus and membranes should be burned or buried deeply and covered with lime.

If, on the other hand, the afterbirth is retained, feed two tablespoonfuls of Dr. Korinek's Kow Konditioner in hot bran mash twice daily. This will hasten its expulsion. After the afterbirth is removed, flush out the womb with Dr. Korinek's Dip (one tablespoonful dip to one gallon warm water) and repeat as often as is necessary, or until the discharge has been completely controlled. Do not breed cows for at least two months after abortion has taken place. A cow that has discharge from the womb resulting from infectious abortion, should never be bred, because of her infecting the bull and passing the infection on to other cattle which the bull may serve.

The preventative treatment of the bull when affected with infectious abortion consists of syringing out the sheath and washing the surface around the sheath with a solution of Dr. Korinek's Dip (one tablespoonful dip to a gallon warm water) three or four times daily, or as often as is necessary, and when the bull is used for service he should be treated before and after service. This will be necessary where infectious abortion has once existed to keep from further spreading the dreaded diseage.

TREATMENT OF INFECTIOUS ABORTION. Consult
COMPANY.

\section{Loss of Appetite (Anorexia)}

CAUSE. This condition cannot always be traced to a definite cause, but is usually due to poor food or weakness of the digestive organs.

SYMPTOMS. Variable appetite; overeats one day and refuses food the next; licking the ground; eating manure, etc.

TREATMENT. Give Dr. Korinek's Physic Capsule and follow with Dr. Korinek's Kow Konditioner; this will improve the general health and restore the appetite. Feed good rich food and plenty of salt.

\section{Loss of Cud (So-called)}

So-called Loss of Cud is merely a cessation of rumination which invariably accompanies loss of appetite in cattle. Consequently one of the first noticeable symptoms in disease of any kind, is that the cow stops chewing her cud, and it is useless to attempt to restore it by giving artificial cuds. When the animal regains her health, the process of rumination is resumed. 


\section{Leeches; Blood-Suckers}

Leeches which suck blood of cattle are sometimes taken up by cattle when drinking water from ponds, etc. The leeches attach themselves to the inner surface of the mouth or nose, and sometimes reach the upper part of windpipe or the gullet. Bleeding at the mouth or nose may be noticed, the membranes where the leeches attach themselves become congested and swollen, and as a result of the loss of blood an anaemic condition follows.

TREATMENT. If the worm can be reached it is easily destroyed by cutting it in two with a pair of scissors or it may be removed with a pair of forceps or with the fingers after wrapping a piece of cloth around them so as to prevent them from slipping.

Steam from boiling hot water containing pine tar or oil of turpentine may cause worms to release their hold if they cannot be removed by other means. In ponds where leeches are numerous, eels should be introduced as they feed upon blood-suckers of this species.

\section{Loss of Cud, or Indigestion}

Loss of cud is not a disease in itself but is one of the first signs of indisposition. Indigestion is usually mistaken for this condition, and some stockmen still believe that a cow really loses her cud and that if she could be induced to chew a handful of hay, she would soon be well again. This is not the case however; the cow is sick, probably from indigestion, and she will not eat until the cause of the trouble is found and removed.

Indigestion is a weakened state of the organs of assimilation which become sluggish and incapable of digesting the food.

CAUSE. Overfeeding; too much bulky or indigestible food, or too dry with insufficient amount of water; moldy forage, frozen roots, etc.

SYMPTOMS. Animal stands quietly and does not chew her cud; the hair is rough; the nose dry, indicating fever; breathing is usually faster than normal, with perhaps a slight grunt. The secretion of milk is suddenly diminished, and if the hand is pressed in the region of the flank and quickly removed, a marked depression or pit will remain which shows that the paunch is full of undigested food.

TREATMENT. Give two of Dr. Korinek's Physic Capsules at once, and follow with one of Dr. Korinek's Colic Capsules morning, noon, and night until well; the latter is a stimulant and will assist the action of the physic. In addition to the above treatment see that the cow is blanketed and her surroundings clean and comfortable.

After treatment give Dr. Korinek's Kow Konditioner in large doses as directed upon the can; this will bring back the flow of milk quickly and prevent further derangements. 


\section{Lung Fever (Pneumonia)}

Inflammation of the lungs may affect one or both sides.

CAUSE. Due to exposure when warm; drenching with irritating medicines when the drench enters the lungs; inhaling smoke, gases, etc.

SYMPTOMS. Unlike the horse, cattle will lie down when suffering from lung fever. Appetite is impaired; breathing quick and shallow; fever high, temperature 105-106 F.; nostrils dilated; nose hot and dry; tongue sometimes hangs out; the elbows are spread to give room to the lungs; breathing causes extreme pain.

In the second stage of the malady, the temperature drops two or three degrees, breathing becomes more painful, milk and all other secretions are suppressed; legs, ears, horns and tail are cold, and if the ear is placed to the side of the chest, a wheezing sound is heard. If the lungs could be examined at this stage they would prove to be hepatized, or resembling in substance the liver.

In the third stage the animal shows signs of improvement. The appetite returns, cough becomes loose, and a healthy discharge flows from the nostrils.

TREATMENT. Place the animal in dry, comfortable quarters; plenty of fresh air but no drafts. Give Dr. Korinek's Distemper Remedy, one tablespoonful every two hours until relieved, then give as often as necessary; if the fever is high, Dr. Korinek's Fever Capsule should be given. Inhalations of medicated steam is also beneficial, using two tablespoonfuls of turpentine to five gallons of boiling water. Supply plenty of fresh water and feed laxative food, such as bran mash; these should be boiled and allowed to cool till about the same temperature as the body. Dr. Korinek's Kow Konditioner will materially hasten recovery.

\section{Laceration of the Eyelid}

CAUSE. Eyelid may be torn on the manger, rubbing-post, wire fences, etc.

TREATMENT. If torn, it may be necessary to take a few stitches in the wound, first washing thoroughly with Dr. Korinek's Dip, one teaspoonful in a pint of warm water, being careful not to get the solution in the eye. If stitches have been taken, they should be removed after the parts have united, and the eye kept clean.

\section{Measley Beef}

Is produced by a larva of common tapeworm of man. These small tapeworm cysts (tænia saginata) are about the size of a pea and found in the flesh of cattle, which become infested by eating food or drinking water which has been contaminated by the feces of persons harboring adult tapeworms. Then again, the person 
becomes infested by eating rare flesh of cattle infested with the-larva stage of measley beef. Great care should be exercised to prevent cattle from becoming infested with this parasite, as person's feces should not be placed where they will infect food or drinking water that is consumed by cattle.

\section{Milk Fever (Parturient Apoplexy)}

A disease peculiar to cows and follows calving, heavy milking cows being most susceptible to it.

CAUSE. Keeping the cow in a poorly-ventilated stable and at the same time feeding too well.

SYMPTOMS. A day or two after calving, a sudden dullness and languor is noticed; an uneasy movement of the hind legs and tail; the cow droops her head and hangs back in the stall; if made to walk she steps uneasily or staggers; pays no attention to her calf. She finally becomes so fatigued that she falls and is unable to rise; the pupils of the eyes are fully dilated and the membranes reddened or congested. The cow may lie on her breast-bone or flat upon the side, most likely the former with her head turned toward the flank, and has the appearance of being sound asleep; the eyeball is not sensitive to the touch of the finger nor will she respond to a pin prick on any part of the body; the temperature in most cases is subnormal.

TREATMENT. If the cow is lying flat on the side, prop her up on her breast-bone; give her plenty of clean dry bedding, and if she is cold, blanket her. Purchase Dr. Korinek's Milk Fever Apparatus and follow directions closely; 95 per cent of the cases recover with this treatment.

\section{Navel-Ill}

CAUSE. This disease affects young calves, the germs finding entrance through the umbilicus of the newly-born calf; the navel at birth being freshly severed and coming in contact with the dirty stall or ground, there is every chance of infection.

SYMPTOMS. There may be swellings of the joints which may burst and discharge pus. This condition may not show itself until the calf is two or three weeks old.

PREVENTION. Same as for prevention of White Scours in Calves; bathe the umbilicus in equal parts Dr. Korinek's Dip and Disinfectant and warm water. (Refer to page on White Scours.)

TREATMENT. In addition to the above treatment for prevention, use Dr. Korinek's Calf Scour and Cholera Remedy as directed on can. 


\section{Physiology of Rumination}

The cow when eating hay or grass, merely moistens it with saliva and swallows it, when it passes into the rumen or paunch which acts as a storehouse for food. After this stomach is filled, the cow finds a comfortable place to lie down and regurgitate the food in small pellets, chewing it finely. When swallowed the second time the food passes into the second stomach for further digestion and assimilation.

It will readily be seen from the above, that the digestive system of the cow is easily deranged, especially from eating too much dry feed.

\section{Paralysis}

May follow calving or Milk Fever and affects both well-fed and poorly-fed cattle; quite common just before calving.

SYMPTOMS. Cow lies down and is unable to rise, although apparently well in other respects.

TREATMENT. Give two of Dr. Korinek's Physic Capsules, and use Dr. Korinek's Milk Fever Apparatus in the same manner as for Milk Fever. Also give Dr. Korinek's Kow Konditioner in doses of two tablespoonsful, morning, noon, and night; feed hot bran mashes and other easily-digested food. It may be necessary to use slings to raise the cow to her feet.

\section{Sore Throat (Laryngitis)}

Inflammation of the membrane lining the larynx or throat.

CAUSE. Overheating followed by too-rapid cooling; taking the animal from a warm stable and driving against a cold wind; sudden change of weather. Also caused by drenching with irritating medicines or by eating from high mangers, etc.

SYMPTOMS. Coughing; throat may be swollen and the breathing difficult; head is stretched out and the nose elevated to facilitate breathing; usually a discharge from the nose.

TREATMENT. Give one tablespoonful of Dr. Korinek's Distemper Remedy three times daily, or in severe cases every three hours as directed on the can. Feed soft foods that are easily swallowed and laxative in nature, such as sloppy bran mash.

\section{Ringing the Bull}

This should be done before the animal is too large and strong. An incision is made in the septum nasi (partition between the nostrils) with a trocar or knife, and the ring inserted and joined; the cut should be made far enough back so the ring will not pull out. If a knife is used for the operation, it should be one with a straight blade that cannot close on the hand. If the animal is very large and strong, he should be placed in a starichion and securely fastened with a rope about the horns or neck, before the operation is attempted. 


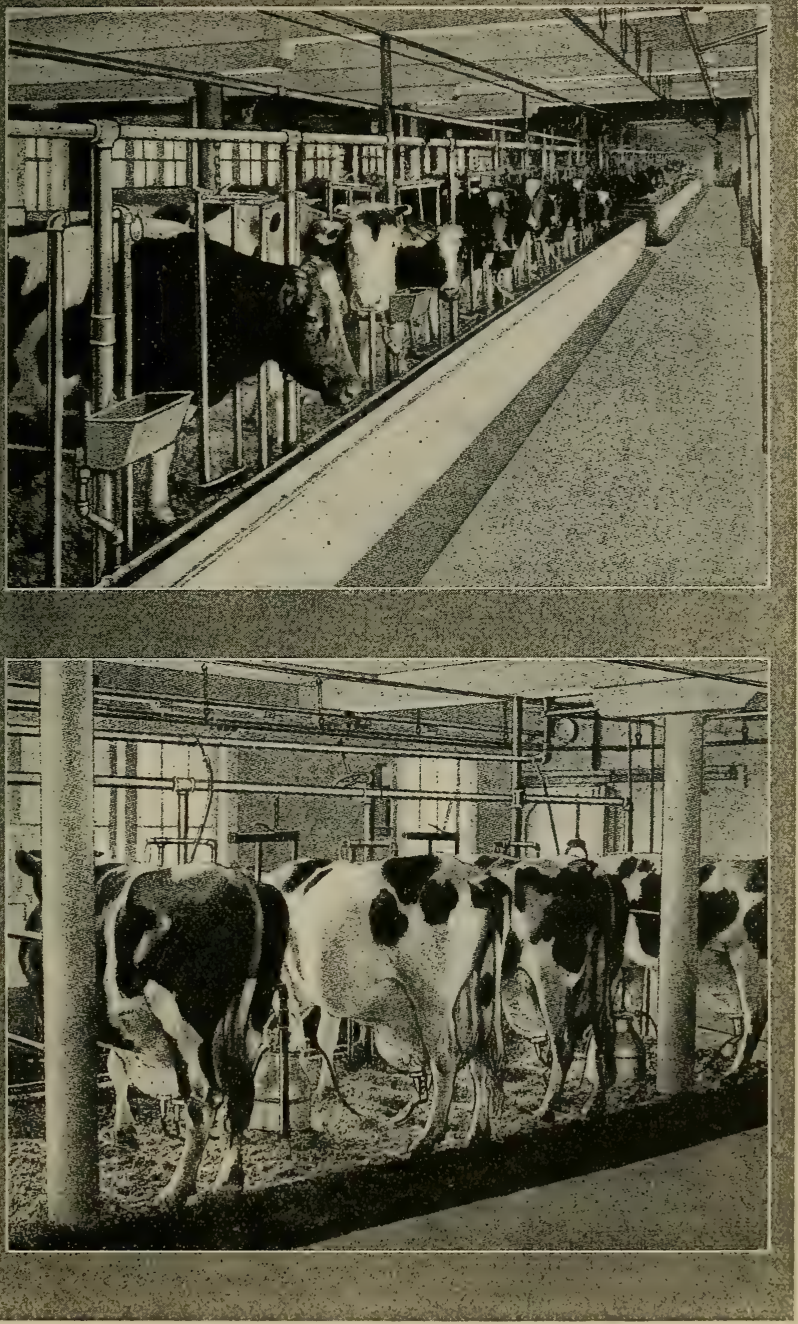

INTERIOR OF MODERN DAIRY BARN 


\section{Round Worm}

CAUSE. An animal swallowing the eggs of the parasite in food or water which has been contaminated with the feces of infected cattle. There are two species, the large round worm measuring from five to fourteen inches in length, the other small round worms varying in size from one-fourth of an inch to two inches in length. Both the small and large round worms infest the intestines of cattle and calves. These worms, especially small round worms, irritate the mucus membrane lining of the intestines, which may cause severe inflammation.

SYMPTOMS. Anemia, appetite variable, diarrhoea, general weakness, dullness and excessive thirst; also, a paleness of the visible membranes of the mouth, nose and eyes. Worms frequently pass with the feces and can be readily seen by a close observer.

PREVENTATIVE TREATMENT. See prevention of Twisted Stomach Worm.

MEDICAL TREATMENT. Give Dr. Korinek's Calf Scour and Cholera Remedy as directed.

\section{Rupture (Abdominal Hernia)}

CAUSE. This disease occasionally occurs in calves from receiving blows from the cow's horns on the right flank. After such an accident a swelling forms near the last rib. This swelling may be neither hot nor painful and is soft to the touch. It can be made to disappear by careful pressure when the sides of the rupture through which it has passed can be felt. On removing the pressure the rupture soon regains its swollen appearance. Similar conditions may also occur in aged cattle, usually due to injuries, such as being kicked by a horse, etc., or due to a weakness of the muscles that are ruptured sometimes during difficult birth.

TREATMENT. Feed the animal on laxative food and feed sparingly on bulky food such as hay, straw and grass. Round the edges on a block of wood which is a little smaller, but the same shape as that of the rupture. After wrapping with cloth, place it over the rupture, then place a bandage around the body. This permits the ruptured muscles to grow together, providing the animal is properly dieted as stated above.

Sometimes a rupture of long standing or a newly produced rupture may be treated by injecting strong solutions of common salt around the torn edges of the muscles. This causes swelling and inflammation which respectively forces the protruding intestines back and closes the opening. There is some danger attached to this method of treatment, and if it is attempted, I would secure the services of a competent veterinarian. 


\section{Rheumatism}

Constitutional disease due to a specific condition of the blood.

CAUSE. Exposure to wet or cold.

SYMPTOMS. Stiffness in gait, variable appetite, constipation, great thirst, hair unthrifty looking; passage of urine is scant; the cow may have fever and usually lies down the greater part of the time. There are two forms of rheumatism, Muscular and Articular; the former affects the limbs and other parts of the body, while the latter attacks the joints.

TREATMENT. Place the animal in warm, dry quarters with plenty of good clean bedding. Feed easily-digested food and an abundance of pure cold water. Give one tablespoonful of Sodium Salicylate three times daily, and if the bowels seem sluggish, give one of Dr. Korinek's Physic Capsules occasionally. Dr. Korinek's Kow Konditioner is an excellent regulator of the bowels for cattle.

\section{Red Water (Bloody Urine)}

A very common condition among cattle in certain districts where pastures are low and poorly drained and sometimes cattle on hilly lands are affected.

CAUSE. Certain kinds of vegetations such as oak shoots, ash, hazel, hornbean and other resinous plants.

SYMPTOMS. In the first stages, the cow appears well except for the color of the urine, but soon a loss of flesh is noticeable and the production of milk is decreased. The milk may be foamy or even tinged with blood. The urine contains no clots of blood but is merely red in color.

TREATMENT. Give two of Dr. Korinek's Physic Capsules, also Dr. Korinek's Kow Konditioner, two tablespoonsful morning and night. Change pasture and feed rich and laxative food.

\section{Retained Afterbirth}

The womb of the cow contains from eighty to a hundred small button-like bodies (colyedons) to which is attached the afterbirth. This accounts for the Retention of the Afterbirth being so common in cows.

SYMPTOMS. The afterbirth hangs pendulous from the cow and if left for some time has a fetid odor. If the cow is unable to expel it, it should be removed at once; if left too long it decomposes and there is danger of the system absorbing the poison from the putrid matter, causing diminution in the secretion of milk, inflammation of the womb, and blood poisoning. 
PREVENTION. Dr. Korinek's Kow Konditioner is a wonderful preventative; give one tablespoonful in the feed, morning, noon and night for three weeks before calving.

TREATMENT. Give two tablespoonfuls of Dr. Korinek's Kow Konditioner in a hot bran mash, morning, noon and night; take the chill from her lrinking water. The afterbirth should not be pulled away by force, as it may tear, leaving small parts which, if not removed, will result in Leucorrhea (Whites) and may terminate in Inflammation of the Womb. To remove the afterbirth insert the hand and carefully detach it from the walls of the uterus, after which wash out the uterus with a solution of Dr. Korinek's Dip, a tablespoonful to a gallon of warm water; an instrument for injecting the solution may easily be made from a section of ordinary hose and a tin funnel. (Refer to cut.)

\section{Ring Worm}

CAC'SE. An infectious skin disease caused by a vegetable parasite (Tenea Tonsurans) and may be transmitted from one animal to another and even to man.

SYMPTOMS. Disease usually appears in the spring of the year and is more apt to affect calves and young cattle. Circular spots of a silvery color appear on the head and neck; the hair falls from the affected parts and the spots become crusty. The infection is thought to be carried by rats and mice.

TREATMENT. Pemove the crusts with soap and warm water, and apply Tincture of Iodine pure to the denuded spots. Disinfect the barn and rubbing places with Dr. Korinek's Dip and Disinfectant as directed upon the can.

\section{Stringy Milk}

CALSE. Due to fungi; cows become infected while wading in stagnant pools or standing in muddy water.

SYMPTOMS. The milk, when first drawn, seems normal, but after six hours or more, a scum appears on the surface which, under a magnifying glass resolves itself into myriads of fine threadlike filaments.

TREATMENT. If cows have been allowed to stand in or drink from stagnant pools, change the water supply; also change the location of the milk house or thoroughly disinfect the surroundings. Give Dr. Korinek's Kow Konditioner-one tablespoonful morning and night. Dr. Ḱorinek's Kidney Capsules are also beneficial. 


\section{Scum on the Eye}

CAUSE. May be due to injury. The eye appears smoke-colored.

TREATMENT. Apply Dr. Korinek's Eye Remedy as directed; two or three applications will remove the scum.

\section{Suppression of Milk}

CAUSE. Usually due to ill health, debility, emaciation, chronic diseases of the bag and poor food. Excitement will sometimes cause a temporary suppression of milk in a nervous cow.

TREATMENT. If due to poor food, improve the quality of the food; if the bag is hard, massage and apply Dr. Korinek's Liniment. Dr. Korinek's Kow Konditioner will increase the milk supply unless the cow has a chronic disease, and if she is diseased she should be examined by a competent veterinarian, as her milk may be unwholesome.

\section{Whites (Leucorrhea)}

Disease common to cows characterized by a discharge of white pus from the womb.

CAUSE. Due to retention of the afterbirth, injuries inflicted by instruments in difficult calving, or may follow inflammation of the womb.

SYMPTOMS. A thick white discharge from the womb is noticeable a week or two after calving, which soon becomes tinged with blood. Cow is unthrifty, loses flesh, and shows a decrease in the milk supply.

TREATMENT. Treatment should begin at the first symptom of this disorder. Give one of Dr. Korinek's Physic Capsules; also give injections of Dr. Korinek's Dip (one tablespoonful to a gallon of water) three times daily, being careful to cleanse the womb thoroughly. Feed laxative food. Dr. Korinek's Kow Konditioner, given according to directions, will quickly restore the milk supply and improve the general health.

\section{Warbles (Grubs)}

A slight elevation of the skin with a grub imbedded within.

CAUSE. The Heel Fly or Warble Fly deposits its eggs on the legs of cattle during the fall months, and the animals licking the parts, take the eggs into the mouth and migrate into the gullet, where they are hatched. They burrough through the tissues and in the early spring will be found in the region of the back in the form of small lumps under the skin. 
SYMPTOMS. These Warbles are seen under the skin in the region of the back and over the loins and are very tender to the touch. When they are fully developed, the larvae work their way out through the skin. This usually occurs in the early part of the summer.

TREATMENT. Use Dr. Korinek's Fly Dope on your cattle and get rid of the flies and the animals will be free from grubs. When the grubs are ripe, or ready to come out, they should be pinched out and destroyed. If they are not ready to come out, inject a little oil with a hypodermic syringe which will kill the grub, when it may be squeezed out.

\section{Tuberculosis}

CAUSE. The bacillus of Tuberculosis thrive in animals, especially those when in a weakened condition or when exposed to atmospheric changes, unwholesome food, dark and poorly ventilated stables. They gain entrance into the body through the lungs or the intestinal canal. They lodge in various portions of the lungs or intestines and multiply very rapidly, causing irritations and formations, nodules, cysts or abscesses. They are the means of the bacillus entering the blood, which carries the infection to other parts of the body, as the spleen, liver, udder, womb, etc. Cows affected with localized tuberculosis, that is to say, the infection is confined to a very small portion of the lungs or any of the above mentioned organs, etc., may give birth to a calf having general tuberculosis at birth or shortly after, due to the cow's blood circulating through the body of the calf before birth.

SYMPTOMS. This disease may pass a casual observer unnoticed, although in some instances we notice a slight cough, unthriftiness, dullness. The coughing is best marked after taking a drink of water in the morning and then being exercised. Some animals keep up in good condition and look perfectly healthy, while some get emaciated, have constipation, variable appetite, and sometimes growths or abscesses can be felt or seen in the udder or glands of the body and neck.

However, cattle showing any weakness or the above symptoms should be tested for tuberculosis by a competent veterinarian who has had the privileges of a veterinary education and experience in the administration of tuberculin.

TREATMENT. It is not advisable to treat tuberculosis. Thus far medicine has failed to relieve the affected animal or kill the bacillus of tuberculosis in a living animal. The infected animals should be disposed of on account of tubercular cows giving birth to tubercular calves, the milk being unfit for human consumption, unless it is thoroughly pasteurized. Infected cattle should be separated from healthy ones, as the disease spreads very rapidly. Drinking and feeding troughs are a means of spreading the infection along, therefore, suspected cases of tuberculosis should be tested 
and if the animals react they should be slaughtered, and if the disease is localized, passed for human consumption. Animals suspected of having tuberculosis or reacting from tuberculin test, their meat should be well cooked.

\section{Ticks}

Ticks are very difficult to kill on account of their protected location, as ear ticks are not affected by dipping, and remedies strong enough for this purpose are liable to injure the animal, but these parasites may be expelled by pouring into the ear, carbolized sweet or cottonseed oil, with favorable results.

\section{Texas Fever (Southern Cattle Fever)}

This disease has various names, according to the locality in which it appears, among them: Spanish Fever, Red Water, Black Water, Red Murrian, Australian Tick Fever, etc.

CAUSE. Due to micro organism (Piroplasma Bigenium) which imbeds itself in the red corpuscles of the blood. The disease is scattered by means of a tick which drops from the affected animal.

SYMPTOMS. Loss of appetite; the animal does not ruminate. There is every sign of unthriftiness and a high fever. When standing, the back is arched; the affected animal seldom stands, however, preferring to lie down most of the time and shows a desire for solitude. The urine is dark or coffee-colored, hence the name "Black Water." The disease is usually fatal, the animal dying within a few weeks.

TREATMENT. Give quinine in large doses. This is considered by all authorities the best treatment. In addition to treatment it is necessary to use every precaution to prevent the spread of the disease, by dipping the animal as well as by thorough disinfection of the premises.

\section{Twisted Stomach Worm}

CAUSE. Cattle become infected with this worm by grazing on pastures on which infested cattle have grazed and scattered their droppings. The worms in the stomach produce a multitude of eggs of microscopic size, which pass out of the body with the feces. In warm weather these eggs hatch in a few hours. The eggs are also destroyed by dryness, but on the other hand, moisture, if the weather is warm, favors their development. The twisted worm measures onehalf inch to one and one-half inches in length.

SYMPTOMS. General weakness, loss of flesh, anemia, dullness, capricious appetite, excessive thirst, paleness of the skin and mucous membranes of the mouth and eyes, and dropsical swelling especially 
that of the lower jaw. Diarrhoea always accompanies this condition and if the feces is carefully examined the small worms may be seen wriggling about like little snakes, or when an animal dies and the fourth stomach is opened these worms can be seen in large quantities.

PREVENTION. Preventative measures are important, as damp, marsh soil favors the development of the embryos. High sloping ground is preferable for pasture. If low ground is used it should be properly drained; burning over the pasture will destroy most of the young worms on the grass and on the ground. Cattle should be supplied with water from flowing streams or wells and not stagnant ponds.

MEDICAL TREATMENTT. Dr. Korinek's Calf Scour and Cholera Remedy as directed.

\section{Tapeworms}

CACSE. Small portions of tapeworms, consisting of one or more segments, are occasiunally seen in the droppings of infested cattle, the infection is undoubtedly taken in with the food or water, infection being spread by the eggs of the parasite, and being expelled with the feces of an infested animal. The eggs being swallowed by insects, worms or snails, which act as an intermediate host, and which when swallowed accidentally by cattle while grazing or drinking carries with it into their stomachs the infectious stage of the tapeworm. Aged cattle do not seem to suffer much from tapeworms, but in calves these parasites cause scours and rapid emaciation.

SYMPTOMS. Emaciation, diarrhoea, loss of flesh, ravenous appetite, paleness of the mucous membranes of the mouth and eyes and the segments of the tapeworms can occasionally be seen in their droppings.

TREATMENT. Dr. Korinek's Calf Scour and Cholera Remedy as directed.

\section{Verminous Bronchitis (Lung Worm)}

CAUSE. Due to worm or parasite called strangylus micrurus, a small thread-like worm two to four inches in length, found in the bronchial tubes, a portion of the lungs. The life history of this parasite is not known, but infection is apparently derived through the medium of pastures where infested cattle have grazed. Young cattle are more seriously affected than old animals, especially common in low marshy pastures.

SYMPTOMS. This form of bronchitis usually affects the entire herd, the animals become poor, unthrifty, hacking, coughing, especially at night and sometimes animals actually cough up worms. 
TREATMENT. Various treatments have been recommended for Verminous Bronchitis or lung worm, as injecting turpentine into the windpipe or fumigating animals by placing them in a closed shed or barn and then burn sulphur, compelling the affected animals to inhale the fumes. This treatment perhaps is the safest and the most effective. A person should remain in the enclosed shed and when the fumes become so strong that there is danger of suffocation, open the doors and windows. This treatment should be repeated every week, until coughing ceases.

\section{Wolf in the Tail}

So-called "Wolf in the Tail" is not a disease, but is merely a relaxed and softened condition of the tail near its end, and may be found in well-kept and perfectly healthy cattle. Hence the fallacy of the belief entertained by some that by splitting the tail near the end with a knife and filling the cut with salt and pepper and bandaging with a cloth, the condition may be relieved.

\section{Warts on the Teats}

TREATMENT. To remove warts, use sharp scissors; the parts should first be washed with an antiseptic solution and the scissors sterilized. After removing the wart, cauterize the wound with Caustic Potash or a Silver Nitrate Stick; a second application may be necessary. 


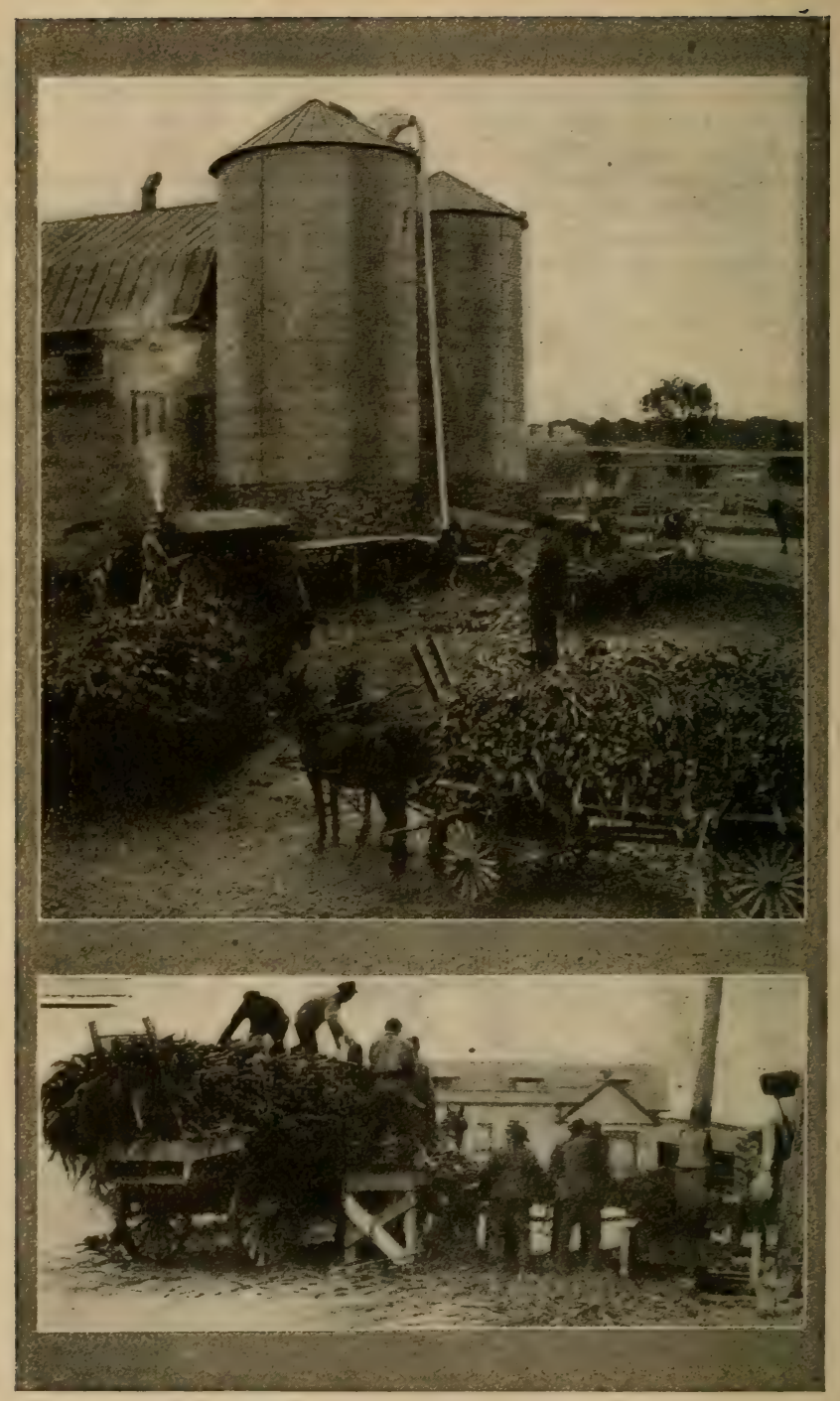

TAKING CARE OF $\cong$ HE FORAGE - 


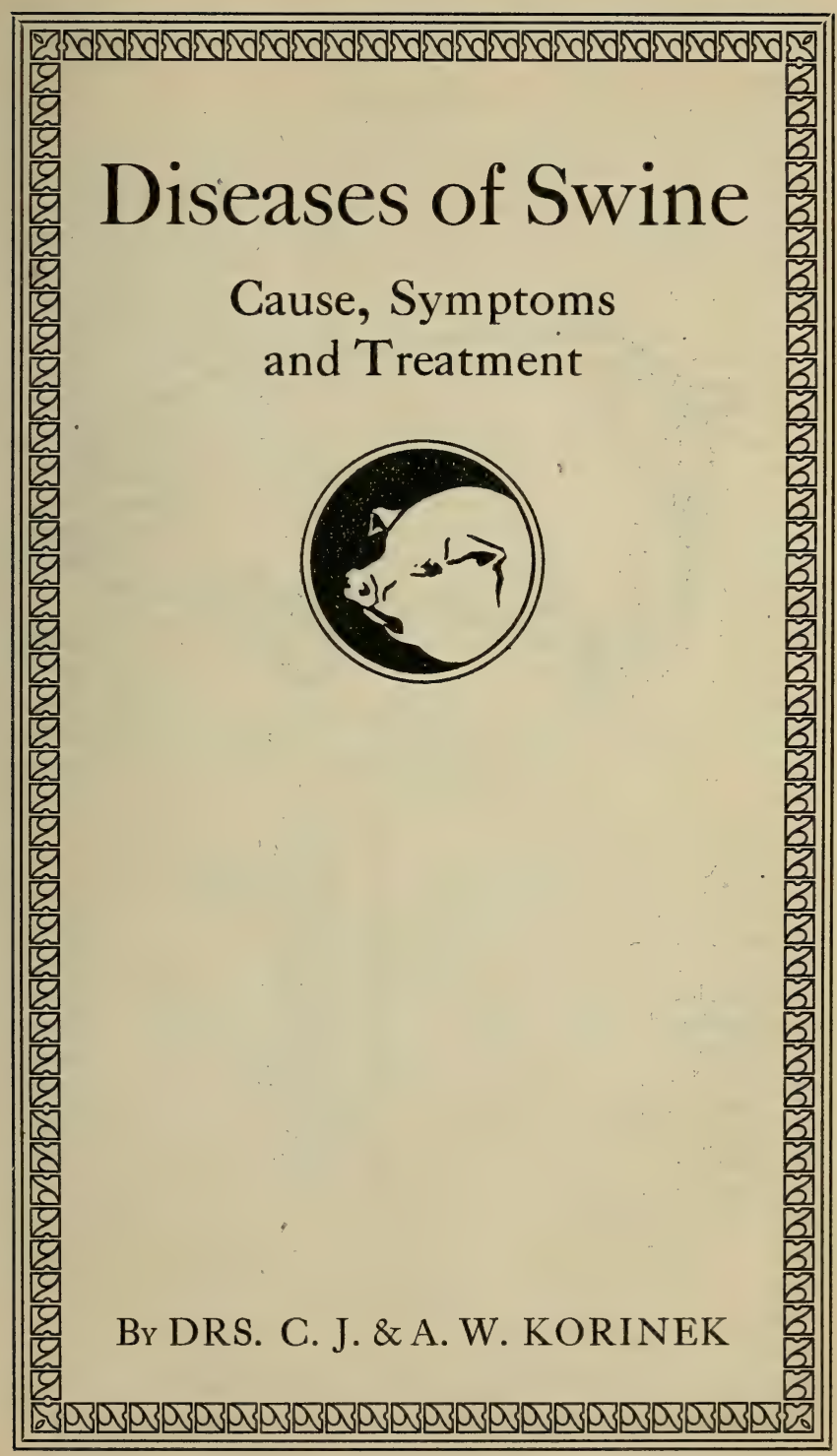




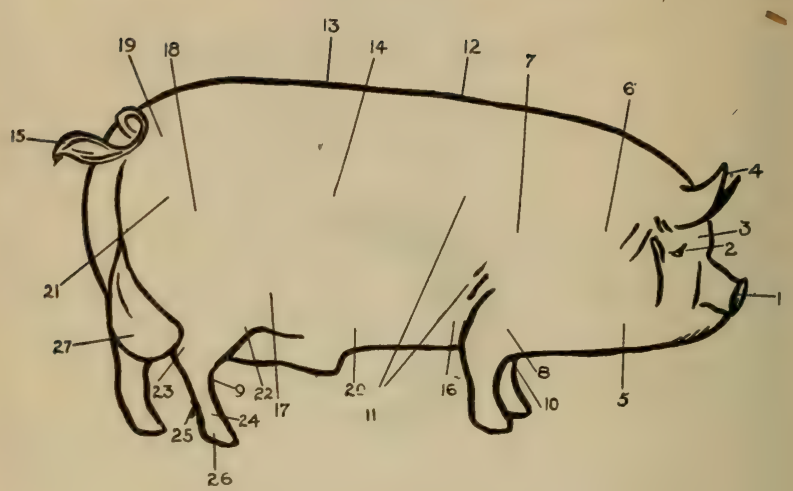

POINTS OF THE HOG

$\begin{aligned} 1 & \text { Snout } \\ 2 & \text { Eye } \\ 3 & \text { Face } \\ 4 & \text { Ear } \\ 5 & \text { Jowl } \\ 6 & \text { Neck } \\ 7 & \text { Shoulder } \\ 8 & \text { Foreleg } \\ 9 & \text { Hindleg } \\ 10 & \text { Breast } \\ 11 & \text { Chest Line } \\ 12 & \text { Back } \\ 13 & \text { Loin } \\ 14 & \text { Side }\end{aligned}$

15 Tail

16 Fore Flank

17 Hind Flank

18 Hip

19 Rump

20 Belly

21 Ham

22 Stifle

23 Hock

24 Pastern

25 Dewclaw

26 Foot

27 Pouch 


\section{Abortion}

CAUSE. Sows may abort at any state of pregnancy by slipping, falling, kicks, or by being caught while crawling through or under fences. Sows may also abort when allowed to crawl in quarters where there are other hogs. Contagious diseases also produce abortion, such as Cholera. Black pleuropneumonia is also a contagious form of abortion in sows, but this is very uncommon, as the disease spreads very slowly.

SYMPTOMS. There is no warning given as a rule, the sows expel their pigs before any signs of abortion are noticed.

In other cases the sows refuse to eat, they get uneasy, shivering and trembling of the muscles and the straining and labor pains are noticed. As a rule when a sow is injured she will not prepare a bed as she would normally.

TREATMENT. Preventative is the only safe and sure treatment. Although when the first sign of abortion appears and there are no signs of the membranes coming away, remove the sows to quiet, warm, clean quarters, off to themselves, and if straining, give one dram of Chloral Hydrate in their drinking water every two or three hours.

When a sow aborts burn the pigs in afterbirth and disinfect the pens with Dr. Korinek's Disinfectant. Keep this up for several days and do not breed until all. discharges from the vagina have ceased flowing.

\section{Administration of Medicine to Hogs}

This may seem easy, but, nevertheless, it is a difficult task. Never lay a hog on his back to drench him. In so doing there will be much danger of strangling to death. The proper method is to stand or set him on end, holding him up by the ears, and by the use of a bottle with a piece of hose drawn over its neck, give the medicine very slowly, so as not to allow a large quantity to accumulate in the mouth or throat at one time. There is always danger of some of the liquids escaping into the lungs and causing them to strangle, or it may produce pneumonia, but this perhaps is the best method of giving hogs medicine by force.

Hogs will generally take the medicine in their feed or drinking water, unless they are very sick or the medicine is extremely disagreeable to the taste.

\section{Blood Poisoning (Pyaemia Septicaemia)}

CALSE. Due to the toxic substance produced by germs that invade wounds, bruises, abscesses or womb following farrowing, if lascerated. 
SYMPTOMS. The seat of injury becomes swollen, pus may adhere to the hair, temperature elevates, appetite poor, moves about very slowly, becomes separated from the rest of the drove, lies around in some cool, quiet place, eventually becomes very weak and poor and dies, if good attention is not given the animal.

TREATMENT. Separate from the other hogs in a clean, comfortable place and wash the seat of injury with Dr. Korinek's Dip and Disinfectant. In case of abscess, open them low so as to assure good drainage. Keep clean, cool water before them at all times. Give mashes made from wheat bran and hot water, or any good, substantial food that is easily digested containing Dr. Korinek's Stock Tonic.

\section{Bronchitis}

CAUSE. Lung worms, poorly ventilated sleeping quarters, sleeping in small stacks of manure heaps, over-heated, filthy pens, where the animals inhale irritating gases given off the bodies of other hogs and in filth. Smoke and dust are very common producers of bronchitis.

SYMPTOMS. Breathing fast, appetite poor, slight raise in temperature, coughing. The hog is dull and stupid, refuses food, but drinks water frequently.

TREATMENT. Preventaitve; avoiding the above named causes, but when affected, move to clion, well-ventilated quarters, avoid dust and gases, disinfect bedding and floors with Dr. Korinek's Dip and Disinfectant. Also give large doses of Dr. Korinek's Stock Tonic. Feed vegetables or any easily digested food and hot wheat bran mashes.

In case it is due to lung worms, confint the animal in a close pen a few minutes and permit them to inhale the steam from turpentine and water by placing water and turpeniine in. a tin receptacle holding about two gallons and inserting heated bricks or stones into the solution.

\section{Black Tooth}

CALSE. Black tooth, so-called in swine, is principally due to injuries to the teeth received by chewing hard matter, such as bone, etc. This causes them to decay.

SYMPTOMS. Toothache. Toothache in swine is similar to that affecting man. Loss of appetite, salvation, or slobbering, hanging the head mostly to the side which is affected, loss of fear of man and of offensive breath. If the hogs are fed on strongly acid food for any length of time their teeth may become darl: colored, although the teeth are not materially injured, so long as decayed tooth substance cannot be noticed and while the appetite snd chewing facilities of the hog do not appear to be diminished, no interference will be necessary. 
It is customary with some people to examine the teeth of hogs and if one is found darker colored than the rest it is supposed to be the cause of the hog not doing well, if in a poor condition, and the tooth is hammered off even with the jaw, leaving the broken roots, lacerated gums, and nerves to increase the hog's suffering. If the hog recovers it is considered a sure case of black-tooth.

My advice is, if you are determined to have the tooth out, extract it properly. DO NOT BREAK IT OFF.

\section{Castration}

This is understood by every farmer, yet there are some points many do not know. For instance, you should use an antiseptic solution, as dip, carbolic acid or bichloride of mercury. Wash your hands and knife thoroughly, also the seat of operation and make your incision as low as possible to permit the pus to drain out nicely. If this is not practiced, the pus will become absorbed into the blood, producing blood poison, which may produce death, or at the best, will cause them to become stunted, where, if the operation was performed properly, the pig or hog would thrive, regardless of the shock from the operation.

I may'add that it is much better to castrate pigs or hogs when their stomach and intestines are empty, and it is always good practice to feed laxative and easily digested foods sparingly after this operation.

\section{Choking}

CAUSE. Vegetables such as potatoes, etc., roots, such as carrots, turnips and sometimes pieces of bone or glass lodge in the gullet. Paralysis of the muscular fibres of the gullet is a very common cause of choking in swine.

SYMPTOMS. The hog is unable to swallow, producing frothing at the mouth and if the obstruction cannot be dislodged death occurs in a very short time. Sometimes the obstruction in the gullet may be felt from the outside with the hand.

TREATMENT. The administration of small doses of raw linseed or olive oil or lard will assist the dislodging the obstruction. Also, careful manipulation of the gullet from the outside with the hand assists in either forcing it into the stomach or bringing it out through the hog's mouth. If vomiting can be produced, it dislodges the obstruction. If immediate results are not obtained from the above treatments, I would recommend butchering the hog for meat immediately. 


\section{Garget-Bag Inflammation}

CAUSE. Injuries, obstructed tits, accumulation of milk in the sow's bag after the loss of part of or all her litter. Difficult birth, slight wounds in the bag permit invasion of germs and very frequently is the cause of bag inflammation.

SYMPTOMS. Heated bag, swelling in one or more tits. The general body temperature elevated one or two degrees above normal. The sow perhaps refuses her feed, although she will drink water in large quantities.

TREATMENT. Feed soft sloppy food and vegetables. Epsom Salts two or four ounces in milk or feed. It is also well to milk the sow relieving her of the milk by hand three or four times a day. This is very necessary. Camphorated oil is very soothing and I would recommend its use freely only over affected tits.

\section{Diarrhoea in Young Pigs (Scours)}

CAUSE. Decomposed foods, slops, etc., fed to the mothers causing her to give toxic milk. Poorly ventilated, filthy, cold and damp pens, insufficient exercise, lack of sunlight, raising pigs by hand or with other sows.

SYMPTOMS. Frequent movement of the bowels; the passage being of a grayish-white color and the odor very disagreeable. At this stage of the disease reliable remedies must be given or they will die very soon.

The discharge from the bowels becomes very thin, the tail and legs become soiled, loss of appetite, the pigs become weak and dull, hair rough and it is difficult for them to move about. In very young pigs treatment is of little value.

TREATMENT. Remove the cause. Clean the sheds, pens and disinfect, feed good wholesome food and give them clean water to drink. To every ten pigs affected with scours give one teaspoonful of Dr. Korinek's Calf Scour and Cholera Remedy four or five times a day in milk or feed. When the pigs are too young to eat or drink, give the sow nursing them one tablespoonful of Dr. Korinek's Calf Scour and Cholera Remedy. three times a day in her feed, swill or drinking water. See that the surroundings are clean, dry and well ventilated. Permit no drafts, for this is a very important treatment in itself.

\section{Hog Cholera}

CAUSE. By the Bacillus Hog Cholerae, contaminated food, stagnant water, filth, etc., all have a tendency to aid its progress. I have seen farms in sections where cholera was prevalent, not in the least troubled with the malady, which was perhaps due to care- 
ful feeding of clean foods, care in watering, cleanliness about the pens and sheds and disinfecting ocassionally and on the other hand perhaps a most important item was that those hogs received tonics containing worm expellers at least four times a year. Many a case of supposed hog cholera is due to worms irritating and producing inflammation of the intestines followed by diarrhoea. A person not familiar with the disease, calls it "hog cholera." In other cases hogs fed swills from restaurants, hotels, etc., sometimes contain soap, washing powders, small particles of glass, etc., leading a person to think his hogs have cholera, and if a thorough investigation isn't made, it is naturally supposed that they died from hog cholera.

SYMPTOMS. In true hog cholera, the temperature will be elevated two to four degrees above normal. There will be a loss of appetite, vomiting, diarrhoea, although there may be constipation when the hog is first affected. The hog wanders off by itself in some cool, quiet place and lies down. When they walk they will stagger and show great stiffness in their hind parts, due to soreness of the intestines. The hair will have a roughened appearance, the back arched, the eyes inflammed and discharge pus, red blotches will show themselves back of the ears, inside the legs and on the abdomen. At this stage the diarrhoea is watery, dark and tinged with blood and very offensive in odor, breath is very fast and labored. The hog grows very weak and dies.

PREVENTION. Prevention must always be born in mind. Do not feed hogs anything you have because "it is only a hog." Always feed good wholesome food and give them clean water to drink. Watch the condition of their bowels and regulate them by feeding. Burn manure and bedding and disinfect carefully. Do not permit your hogs to drink out of running streams of water, especially if hog cholera is in your neighborhood. When buying hogs it is well to keep them off by themselves for two or three weeks, as they may be diseased. Do not permit neighbors, their stock or dogs on your premises when hog cholera is raging, as the infection of hog cholera can be spread very rapidly by matter from the affected hogs adhering to the shoes of man, to the feed of stock and dogs, etc.

Disinfect your pens once a week with Dr. Korinek's Dip and Disinfectant, using one part Dip to fifty parts of water; troughs and sleeping places must be disinfected as well, take burlap sacks that have been saturated in a solution of Dr. Korinek's Dip and Disinfectant and wrap it around rubbing posts, etc. It is well to dip your hogs in case you have a vat. Internally give the following: Wood charcoal, powdered, 2 lbs.; sulphur, powdered, 2 lbs.; sodium chloride, powdered, 3 lbs., sodium sulphate, powdered, 3 lbs.; sodium hyposulphite, powdered, 2 lbs.; antimony black, powdered. 2 lbs. Mix thoroughly and give a dessert spoonful to an urdinary size hog, smaller or larger hogs may be dosed accordingly. (See serum treatment for Hog Cholera on page 102.)

I am postive that if this method was properly practiced by all hog raisers and feeders, hog cholera would be a very rare disease. 


\section{Indigestion}

CACSE. Worms are perhaps one of the most common causes. Unwholesome irritating food or swill containing soap or washing powder all have a tendency to derange the process of digestion.

SYMPTOMS. Abdominal pain, vomiting, back arched, breathing rapidly and temperature elevated from two to three degrees. There may be diarrhoea and the animal may be constipated. Vomiting as a rule relieves acute attacks by expelling the irritant eaten. When it takes a chronic form the hogs become stunted.

TREATMENT. Endeavor to find out the cause and remove it. If constipated, give calomel 15 to 20 grains or if diarrhoea appears, give one tablespoonful of Dr. Korinek's Calf Scour and Cholera remedy three times a day in their feed. Always feed unthrifty pigs or hogs Dr. Korinek's Stock tonic; it expells worms and aids digestion. Feed it with wheat bran and hot water to sick animals.

\section{Jaundice or Yellows}

CACSE. Liver flukes, intestinal worms, gall stones, lack of "xercise, over-feeding or a stoppage of the bile duct.

SYMPTOMS. The white portions of the eyes take on a yellow color, also the membranes of the mouth. The back arches, hair looks rough, vomiting, temperature rises, animal is constipated, diarrlosea is sometimes noticed. Urine is passed frequently and is of a dark color.

TREATMENT. This disease requires careful feeding and lots of exercise. Give ten to twenty grains of calomel, then follow with Dr. Korinek's Tonic in large doses. It is doubly indicated in this disease, especially if due to worms. Feed swill and vegetables. Give them all the pure water they will drink.

\section{Kidney Worms}

CALSE. Damp, filthy surroundings seem to favor the growth of the embryo worm. They are taken into the digestive canal with the food and eventually pass to the region of the kidneys where they find conditions favorable for multiplying.

SYMPTOMS. Paralysis of the hind quarters. In paralysis the animal does not exhibit as marked tenderness from pressure of the fingers over the loins, as would be present if the weakness of the hind quarters were due to a sprain or to rheumatism of the loins. Occasionally hogs may suffer from the presence of one or more worms in the kidneys, but the ailment is rarely fatal, only becoming so after a long period of suffering and consequent degeneration 
of one or both kidneys. The presence of the worms in the kidneys is almost impossible to detect except through a microscopic examination of the urine. If worms are found in the kidneys of an animal that has been slaughtered for food, or has died from disease, it may then be supposed that others of the same herd, if not acting in a normal manner, are infected with the same worm and the same disease.

TREATMENT. Teaspoonful doses of turpentine is the only treatment that I can recommend. Preventative measures form the only practical method of treating diseases of this nature.

\section{Kidney Congestion}

CAUSE. Hogs are subject to various injuries about the kidneys, such as a large number of hogs piling up together, also exposure to cold, wet rains.

SYMPTOMS. Small quantities of dark colored urine is passed frequently, appetite poor, no energy to move about. They lie around a great deal; at times they may be paralyzed and dragging their hind quarters.

TREATMENT. Apply cloths or blankets, wrung out of hot water, also give potassium-acetate in 20 grain doses four or five times a day in drinking water. Feed soft, sloppy food containing Dr. Korinek's Stock Food and Tonic at the rate of one tablespoonful three times a day. It contains nerve stimulants, just what is indicated in paralysis.

\section{Lung Worms}

CAUSE. By threadlike worm varying in length from one-half to one and one-half inches and of a brownish-white color. They are found in the windpipe and tubes leading into the lungs. The adult worms in the lungs produce large quantities of eggs which are coughed up with mucous and become scattered over premises where other hogs are permitted to walk. They inhale the dust containing the eggs where they find moisture sufficient for their development.

SYMPTOMS. Severe coughing spells. Large quantities of mucous will escape from the nose and mouth. The hog becomes stunted although he may eat fairly well, but if not relieved, the worms collect in the Bronchi and produce sudden death, due to suffocation. The worms may set up an inflammation of the lining membranes of the lungs which is sometimes taken for Swine Plague or Cholera. This disease is not uncommon, especially in old, filthy, poorly drained hog houses and pastures. 
TREATMENT. Confine the affected hogs to a shed, close windows and doors and any large cracks, then compel them to inhale steam from the following mixture: Turpentine eight ounces, Pine Tar one pint, Water two gallons. Place in tin receptacle in center of the shed and heat the above solution by adding hot bricks or stones to the mixture occasionally. Compel them to inhale this steam for at least thirty minutes twice a day. Feed wholesome food to which add Dr. Korinek's Stock Tonic as directed in the proportion of one teaspoonful to every one hundred pounds of hog weight. A strong, vigorous hog may have worms, but they retain their vitality.

\section{Lung Fever (Inflammation of the Lungs)}

CAUSE. Sudden changes, exposure to storms, piling up of hogs during cold nights or sleeping in manure heaps, old straw stacks, etc.

SYMPTOMS. The pig or hog is taken with shivering spells, is stupid, his back is arched, loss of appetite, temperature elevated two or four degrees above normal, short hurried breath generally accompanied with cough which is deep and hoarse. As a rule the hog is constipated.

TREATMENT. Place in good clean, warm, well-ventilated quarters, free from draughts. Keep water before them at all times containing salt petre, one teaspoonful to every gallon of water. If constipated do not give physics; give injections of soap and warm water; also administer about one-half teaspoonful of Dr. Korinek's Distemper Remedy on the tongue with a wooden paddle. It sticks to the surface of the tongue and gradually dissolves and gives great results, as it is very soothing to the organs of breathing.

\section{Lice on Hogs}

Dip, spray or scrub your hogs with Dr. Korinek's Dip and Disinfectant, but whatever remedy is used should be applied more than once, which, of course, causes considerable work where there is a large number of hogs infested, unless dipping is done. The reason for repeated applications being necessary is that hogs may pick up lice from the ground, bedding and rubbing places, and other reasons are that though the remedy used may kill the lice, they do not affect the nits and these remain intact and hatch within a week or ten days. A new crop of lice appear on the hog from this source. Remove all manure and bedding from pens and sheds and burn it. Disinfect floors and spray sides of shed, pens and rubbing places with Dr. Korinek's Dip and Disinfectant, one part to seventy-two parts of water once a month and you will be handsomely repaid for your labor. 


\section{Mange}

CAUSE. By the Sarcoptes Scabei. This parasite burrows under the outer surface of the skin.

SYMPTOMS. The parasite usually manifests itself on the skin under the arm pits, thighs and inside of the fore-legs. At first small red blotches or pimples appear and these gradually spread as the parasites multiply and burrow under the skin.

TREATMENT. There is no way of curing it or of preventing it, except by killing the parasites and their eggs; not only on the pigs themselves, but also on the sides of the pens, sheds, rubbing-posts, or anything that an affected hog rubs against.

When treating this disease, the real aim must be to kill the parasite by the prompt and continuous use of external remedies, such as washing or dipping, which is better, with Dr. Korinek's Dip and Disinfectant, one part to seventy parts water. Repeat this every ten days until cured. Two dippings are generally sufficient. It is well to feed cooling foods, such as slops and vegetables containing Dr. Korinek's Stock Tonic as directed on package.

\section{Nettle-Rash (Urticaria)}

CAUSE. Irritations to the skin produced by sprinkling hogs with irritating solutions, powders or from irritating dips when treating lice, etc. Feeding highly nitrogenous food predisposes them to this disease; also filth, poorly drained sheds and pens, especially common in young pigs. Nettle rash is not contagious, but what produces it in one hog may produce it in several at the same time.

SYMPTOMS. Red, swollen blotches appear on the skin very suddenly, especially about the ears and the inside of the thighs, perhaps due to the skin being thin and deprived of hair. The hog rubs it on account of the intense itching. The hog will not thrive when in this condition. In most cases there is a fluid oozing from the blotches, causing dirt and filth to adhere to the hair. However, if the disease is properly treated, a recovery is sure to follow in about two weeks.

TREATMENT. Prevention against this disease is a very good treatment, and it consists in keeping shoats and pigs in clean, wellventilated sheds and pens. Do not sprinkle them with irritating solutions or powders or irritating dips, but when the disease once shows itself, give each pig or hog affected a dose of Epsom Salts, one ounce to every twenty-five pounds of hog weight in feed, swill or drinking water. If the weather is hot, keep them in a clean, cool place, also purify their blood by feeding Dr. Korinek's Stock Tonic, one teaspoonful to every twenty-five pounds of hog weight. 
EXAMPLES. If a pig weighs twenty-five pounds, give one teaspoonful twice a day. If they weigh 12 pounds, give one-half teaspoonful twice a day. Larger hogs, give doses in proportion. Apply Dr. Korinek's Dip and Disinfectant, one part to 100 parts of water. It is non-irritating and will destroy hog lice and will heal the pustules of nettle rash. Dip twice, ten days apart. It also must be borne in mind that their pens and sleeping quarters must be disinfected; the old bedding and manure burned and replaced with good clean straw or hay. Feed casily digested food, slops, etc.

\section{Navel Rupture (Navel or Umbilical)}

CAUSE. Injuries. Pigs crowding through narrow doorways or openings in fences, small pigs sleeping with large pigs and allowed to pile up or being thrown about feed troughs when feeding with larger hogs. Weakness and constipation also predisposes them to navel or umbilical rupture.

SYMPTOMS. A soft, puffy swelling about the navel or umbilicus, varying in size from a hazelnut to that of an ostrich egg. When a pig is placed on its back the intestines will gravitate into the abdominal cavity, providing the intestines will not adhere to the walls of the rupture.

TREATMENT. This is most difficult, as often times the intestines will adhere to the inner surface of the rupture and unless the operation is carefully performed, there is great danger. Great care must be exercised in preparing the pig by fasting it for twenty-four hours. After this is accomplished prepare an antiseptic solution of dip-carbolic acid or bichloride of mercury; also have a needle and absorbent silk or cat gut ready. Now, wash the seat of operation with the antiseptic solution. Place the pig on its back with its head downward; make an incision through the skin carefully, as stated before, as the intestines sometimes adhere to the inner surface of the rupture. If such is the case, wash the hands in the antiseptic solution and with the fingers carefully break the adhesions or separate the intestines from their adhesions. After this is accomplished, sew the inner lining of the abdominal cavity with absorbent silk or cat gut. Then sew the outer skin with cotton or linen cord and your operation is complete. Feed the hog sparingly for a few days following the operation on easily digested, laxative foods.

\section{Piles; Prolapsus of the Anus}

CAUSE. Due to irritating foods that either constipate or produce diarrhoea. Although the animal may look well, there wili be a weakness of the circular fibres of the intestines. 

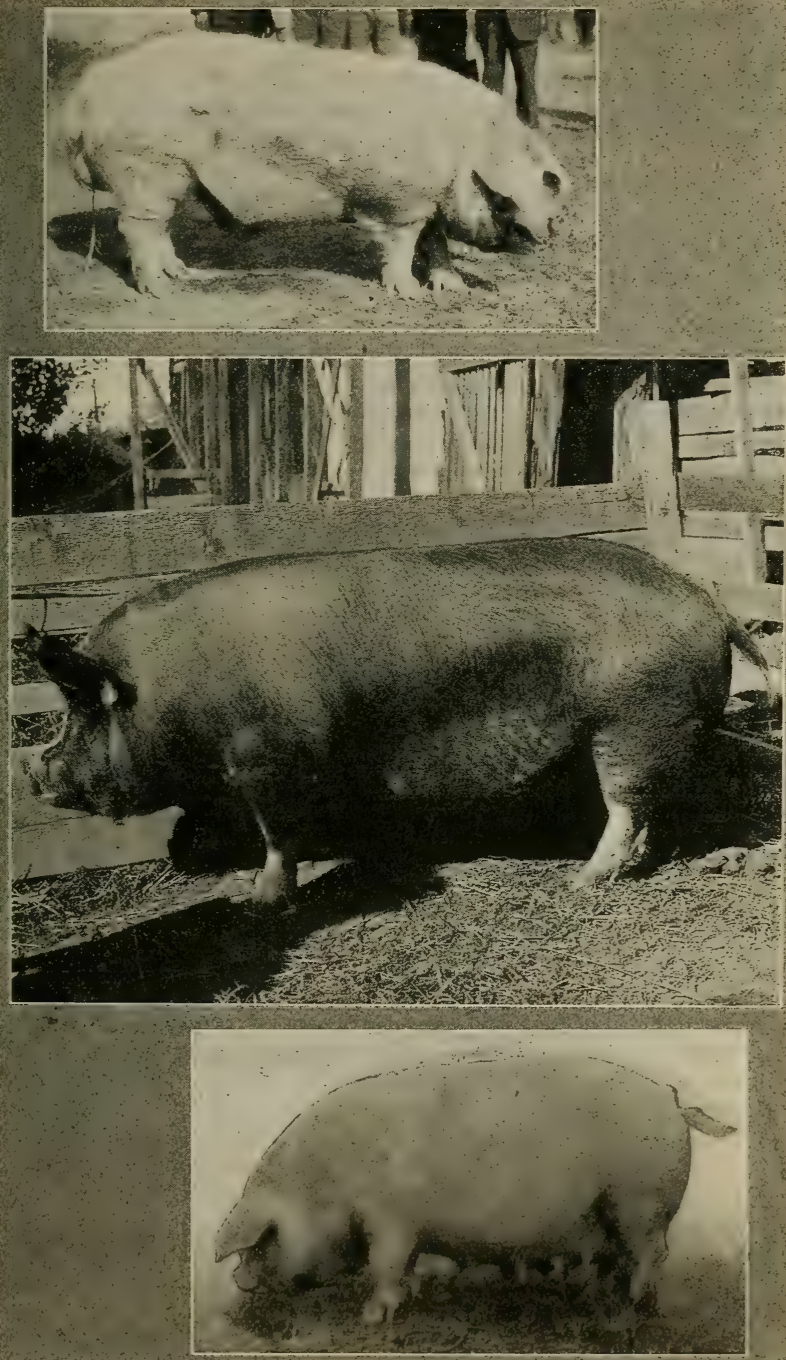

PROFIT MAKERS 
SYMPTOMS. Symptoms are very plain-a protrusion from the rectum from two to four inches in length. This protrusion is irritated by the animal rubbing against the sides of pen, etc.; it cracks and bleeds, and if the weather is warm will become fly-blown and maggots will accumulate in large quantities.

TREATMENT. In the first stages of the disease, wash the protruding parts with an antiseptic solution of carbolic acid, one teaspoonful to a pint of water. Give rectal injections of soap and warm water or sweet oil; give about two ounces of castor oil internally and feed soft, sloppy foods. In chronic cases of long standing, remove the exposed portion of the intestine after washing thoroughly with an antiseptic solution. Remove with sharp knife and stitch the cut end of intestine to the edges of the anus. Feed easily digested foods, such as wheat bran mixed with flax seed meal on which boiling hot water has been poured. Let it stand until its heat can be borne by the hand, before feeding to the animal.

\section{Paralysis of the Hind Quarters}

CAUSE. There are a great many things that may produce paralysis of the hind quarters. For instance, shipping hogs in crates, fractures of thigh bones, Rhachitis, due to feeding food that is deficient in mineral matter, hogs piling up, kicks or injuries to the back frequently seen in sows nursing a litter of pigs while in a rundown condition. Constipation and indigestion also produce paralysis of the hind quarters. Some think it is caused by worms in the kidneys; this is not always the case. It is true that the presence of the parasite around the kidneys may cause irritation of the nerves of the spinal column and result in paralysis, yet, there more often is a weakness and loss of nervous power of the hind parts.

SYMPTOMS. Regardless of the cause, the symptoms in either case are exactly alike, and take the form of weakness of the back, wriggling of the hind parts, and finally they sit down on their haunches. After some effort they get up and run in a straight line quite fast, soon swinging to one side for a while and then go over to the other side and finally get down so that they cannot rise, but can drag themselves about. The appetite is good until a day or two before they die.

TREATMENT. Place the hog in clean, comfortable quarters with plenty of fresh water to drink. Give sour milk, fruit or vegetables containing Dr. Korinek's Stock Tonic as directed on container. It contains nerve stimulants and blood purifiers. If the hog is constipated, add two or four ounces of Epsom Salts to their feed.

Treatment of all such cases requires perseverance and recovery is slow and sometimes uncertain. 


\section{Pin Worms}

CAUSE. Hogs take the eggs that encapsule well matured embyronic worms with their feed or drinking water. They multiply very rapidly in the small intestines and are one-half to one inch in length.

SYMPTOMS. No signs are noticed unless they are very abundant, as they are small and difficult to see with the naked eye. The principal location is in the back part of the small intestines where considerable inflammation is set up, especially where there are other worms present, such as the round worm.

TREATMENT is of little value, as the location in the intestines is very difficult to get at, but as their presence causes very little disturbance it is hardly worth while treating; although preventative measures should be applied by disinfecting, burning manure and bedding.

The following have proven a very effective treatment for Pin Worms: Powdered Quassia one pound, Sulphur Sublime two pounds, Glauber Salts one pound, Powdered Tobacco one-half pound, Sulphide of Antimony one pound, Hyposulphite of Soda two pounds, Beachwood Charcoal one pound, Common Salt two pounds.

The above must be well powdered and thoroughly mixed. Give one heaping teaspoonful to every one hundred pounds of hog weight. To small pigs give doses in proportion to weight, as above stated. Piace it in their feed or slop twice a day. In addition to the vermifuge use an alternative and tonic that should be given pigs and hogs that do not thrive properly. Best results, however, are obtained in treatment of pin worm when the principal food consists of vegetables, mashes and slops.

\section{Pleurisy}

CAUSE. Exposure to cold, damp, chilly weather, especially when exposed to draughts or a large number, of hogs allowed to pile up during cold nights, etc.

SYMPTOMS. Chilled and temperature elevated two to three degrees above normal; breathing fast. The hog will show great pain when pressed over the lungs, by flinching, squeeling or grunting; coughing suppressed, ribs rigid; breathing mostly with the muscles of the flanks; appetite poor and eventually there will be fluids accumulate in the lung cavities. At this stage the breathing is labored and difficult. If the ear is pressed over the lungs the fluids can be heard. When in the first stage, the sound will be similar to that of rubbing hair between the finger and thumb.

TREATMENT. Remove the cause and treatment will be satisfactory if given in due time. Place in clean, comfortable shed; see 
that it is well ventilated, prevent drafts; apply Dr. Korinek's Absorbent Blister over the lungs and give two to four ounces of Castor Oil in milk. Feed easily digested food, such as hard wheat bran mashes containing Dr. Korinek's Stock Tonic. It is also well to feed vegetables.

\section{Rheumatism}

CAUSE. Exposure resulting from cold, damp houses. Overfeeding also has a tendency to produce swelling of the joints and muscles.

SYMPTOMS. Lameness of one or more of the limbs, swelling of the joints about the legs and feet. The animal does not care to move and will refuse his feed in most cases; temperature slightly above normal; breathing quick and short; will drink water frequently if it is offered.

TREATMENT. I am of the opinion that rheumatism in swine would be a very rare disease if they were properly provided for and furnished clean, dry quarters with a liberal quantity of bedding. Do not allow your hogs to pile up in their sleeping quarters, as it is very injurious to them.

Medical treatment consists of feeding Dr. Korinek's Stock Tonic in tablespoonful doses, three times a day in sloppy food; also feed vegetables or green food.

\section{Round Worms}

CAUSE. Is undoubtedly due to filth, or hogs eating food or drinking water contaminated with well-developed eggs or embryos of round worms, thus taking them into their digestive canal where they multiply rapidly and set up considerable irritation. This worm varies in length from three to thirteen inches and is of a reddishbrown color.

SYMPTOMS. The round worm is generally passed with the feces and can be readily seen with the naked eye. A hog infected with a large number of these worms is generally restless, appetite varied. When these worms develop in large numbers they obstruct the intestines. In other cases they irritate and inflame the intestines, causing inflammation and diarrhoea, and death may be due to either obstruction or inflammation of the bowels.

TREATMENT. Treatment is very satisfactory. Withhold all food from 18 to 24 hours. Then place in one pint of finely ground feed: Calomel and Santonin, each five grains to every one hundred pounds of hog weight. For instance, if the hog affected with round worms weighs 200 pounds, double the dose by giving ten grains of each of the above, but if the hog only weighs fifty pounds, give one-half the dose mentioned, or two and one-half grains of each. 
This treatment should be repeated in a week or ten days to assure the expulsion of worms that might have survived the first dose. Feed sparingly on laxative food for a few days following each treatment, as bran mashes and vegetables.

\section{Rupture (Scrotal or Inguinal)}

DEFINITION. In the male the intestines pass through the wide inguinal canal, through which the cord of the testicle passes. It is not difficult to recognize this form of rupture, as the enlarged scrotum on the pig that contains the testicles is usually quite large and sometimes almost reaches the ground. In this case both sides of the scrotum, or the sack which contains the testicles, also contains intestines. In this case the pig is held up by the hind parts, the intestines are gravitated back into the abdominal cavity, but as soon as a pig lies down or stands, they again return to the scrotum. The testicles can be located at the bottom of the enlargement.

CAUSE. Hereditary tendencies predisposes swine to rupture. Pigs with large inguinal canals through which the cord passes and by pigs being crowded, injured, squeezed at troughs or passing through narrow doorways. Weakness is another very common cause; severe straining from constipation also produces rupture.

SYMPTOMS. An enlargement of the sack containing the testicles. Sometimes there may be a strangulation of the intestines where they fold or twist. They become inflamed and produce death. The pig dies in great pain, but fortunately the strangulation of ruptures in pigs is very rare, as the scrotum and canal in which the intestines occupy releases and becomes very roomy.

TREATMENT. Operation is the only method of relieving or curing inguinal or scrotum rupture. My advice is to operate as soon as possible. When the pigs are small there is less danger. The pig to be operated on should be fasted for at least twenty-four hours, as it is easier on both the operator and pig when the intestines are empty, or nearly so.

The operation that I would recommend is as follows, and I have found it to be very successful: First, hang, or have an assistant to hold the pig up by its hind legs, prepare an antiseptic solution of Dip, Carbolic Acid or Bichloride of Mercury in a pan, have a needle threaded with a medium size absorbent silk or cat-gut suture, also have a clean, sharp knife handy; wash the seat of operation with the antiseptic solution. Now, proceed to locate the testicle, by having the hind parts elevated. The testicle will remain in the sack or scrotum; now grasp the testicle between the fingers and make the incision through the sack and to the lower portion. It may be necessary to insert two fingers to withdraw the testicle. When the testicle is located, withdraw it. Before cutting it off, it is well to run a needle containing a thread through the last covering 
of the testicle so as to prevent it from returning inside when the testicle is removed. After this is securely done, withdraw the testicle and tie an absorbent silk or cat-gut just back of the testicle. Remove the testicle and sew the inner membranes that envelop the rupture and testicle with what is called a tobacco pouch suture. Draw it together firmly and tie and cut off suture about one-half inch from the knot.

Now, your operation is complete. Do not sew the outer incision in the scrotum, as it would have a tendency to accumulate dirt and hold pus, which should have a free drainage to escape. Wash with some of the above antiseptics twice daily until thoroughly healed Also, feed laxative foods that are easily digested.

\section{Sun or Heat Stroke}

CAUSE. Hogs that are very fat and driven, hauled or shipped to market when the weather is warm are frequently stricken with heat or sunstroke. Sometimes when hogs are overcrowded and not protected from the rays of the sun or heat may become victims of heat or sunstroke.

SYMPTOMS. First they stagger when walking, then they become very weak and temperature elevates three or four degrees higher than normal. Prostration or extreme depression and sumetimes involuntary spasms or contractions of muscles occur.

TREATMENT. Prevention. Do not drive, haul or ship hogs that are not accustomed to exercise or extreme heat during the hottest part of the day. Do not crowd hogs in small pens or sheds during the hot months, as their bodies give off considerable heat in addition to that of the sun. See that they are protected from the sun. When hauling or shipping hogs, wet them occasionally with water. It prevents heat stroke. In case a hog is suffering from heat or sunstroke, place them in a cool, shady place and apply ice or cold water to the head only. Also, give saltpetre in teaspoonful doses every six hours diluted in one ounce of water. Also give alcohol, one teaspoonful every three hours in one ounce of water. Sometimes good recovery is obtained from the above treatment.

\section{Rickets}

CAUSE. Food deficient in mineral matter or lime, filth, lack of exercise, crowded quarters all tend to produce a softening of the bones and swelling of the joints.

SYMPTOMS. The pigs affected are generally in good condition and seem to be doing well, but all of a sudden they become paralyzed in the hind quarters owing to the weakened condition of the bones, which sometimes fracture without receiving injury or any additional weight to that of the pig itself. The bones of the snout, back, limbs and feet bend and become deformed. The pigs grow weak, poor and stunted and perhaps the best treatment is to destroy them. 
PREVENTATIVE TREATMENT. Careful feeding of good wholesome food. This disease is very seldom seen where hogs are frequently fed corn.

MEDICAL TREATMENT. With the first signs of Rickets, feed Dr. Korinek's Stock Tonic in tablespoonful doses twice daily. It contains the following mineral ingredients: Sulphate of Iron, Lime, Sulphur, Chloride of Soda, Potassium Nitrate, Sodium Bicarbonate, Hyposulphate of Soda.

The above drugs are excellent bone builders. In addition to the above mentioned ingredients, Dr. Korinek's Stock Tonic contains general tonics derived from the vegetable kingdom.

\section{Serum Treatment of Hog Cholera}

This treatment has been successful in some cases and in others unsuccessful. The latter perhaps due to poor serums, or the disease so far advanced that the hogs treated were beyond recovery. Serum treatment is very expensive and requires a strictly septic operation of injecting the serum, so that a hog raiser or grower is not equipped to administer the treatment properly, besides it requires an additional expense added to the cost of the serum, the services of a veterinary surgeon. Therefore, I would use preventative measures as stated previously.

Dr. Korinek's Dip and Disinfectant destroys the bacillus of hog cholera.

Dr. Korinek's Stock Tonic expels worms, tones the system, regulates the bowels and fortifies your hogs against hog cholera.

\section{Sows Eating Their Young}

CAUSE. Insufficient lime or mineral matter fed them prior to farrowing; constipation is also a fruitful cause.

PREVENTION. Careful feeding for a few days prior to farrowing, such as feeding slops free from soap or washing powders, cool food, such as wheat bran mashes with one tablespoonful of Dr. Korinek's Stock Tonic twice a day. It is loosening to the bowels and it also contains mineral matter and blood purifiers which are very valuable in the above mentioned condition.

\section{Sore Mouth}

CAUSE. Feeding decomposed foods. Also slops or stagnant water, washing powders, broken glassware from the tables fed in slops, barley or wheat beards, etc. Difficulty in eating, or they refuse to eat at all. Stringless secretions of saliva continually oozing from the mouth. The mouth gives off very offensive odor. 
TREATMENT. In this form of sore mouth, remove the cause. Feed soft, wholesome food such as wheat bran mashes and vegetables. In cases where it is due to the lodging of beards of wheat or barley, gag the hog's mouth with a piece of wood and remove the beards with forceps. Keep clean cooked wheat before them at all times and avoid feeding dry; hard food.

\section{Sore Feet}

CAUSE. Filth; very common in large hogs when confined to hard floors or driven over rough, hard roads or continually kept in filthy pens. The tissues of the feet become softened, especially those between the claws. Irritation is set up by germs entering the abrasions.

SYMPTOMS. The hog will be noticed going very lame and if closely examined, the above conditions will be found.

TREATMENT. Remove the hogs to clean, dry pens containing plenty of clean bedding and wash the affected parts with Dr. Korinek's Dip and Disinfectant (one part dip and disinfectant to 100 parts water). Repeat this treatment at least once a day. In case the feet are badly inflamed, I would advise the application of hot flaxseed meal to the feet. Feed easily digested food, as it adds materially in the treatment of infectious wounds.

\section{Thorn-Headed Worms}

CAUSE. A form of grub that is found in old manure heaps, straw stacks and hog lots. The eggs containing embryos of the thorn-headed worm is eaten by the white grubs, then the white grub is eaten by the hog. The larva of the thorn-headed worm is liberated by the process of digestion and becomes a parasite of the intestines of the hogs, where it develops into a fully matured worm. Large numbers of hogs quickly become infected with this parasite as they multiply very rapidly. The worms vary from two to twelve inches in length and have a whitish color.

SYMPTOMS. As a general rule a worm can be seen in the feces. Other signs are that the hog loses flesh, appetite irregular, constipation and then again there may be diarrhoea, especially where there are large numbers of worms present.

TREATMENT. First of all burn all manure or decomposed vegetation that the hogs are liable to come in contact with. Withhold all food from eighteen to twenty-four hours and give one teaspoonful of oil of turpentine to every one hundred pounds of hog weight, or if the hog weighs less than one hundred pounds, doses should be given in same proportions as above. Follow this treatment for three or four consecutive days. Turpentine is easily given to hogs as they will drink it in milk when well mixed. Per- 
haps it is advisable where a large number of hogs are affected to divide them into pens of five or ten hogs, as they are less likely to get over-doses. Feed laxative food. Clean and disinfect troughs and feeding floors.

\section{Thumps}

CAUSE. Disorders of the digestive system from overloading the stomach and causing irritation of the nerves leading to the diaphragm, the membrane that separates the lungs and heart from the intestines, stomach, liver and spleen. It is a spasm of this membrane that causes a hog or pig to have "thumps." Insufficient exercise. A large number of pigs may become affected at the same time when closely confined.

SYMPTOMS. Jerking of the flanks; the pig or hog becomes very weak and stunted in a very short time.

TREATMENT. Remove the cause. In pigs, when first affected, careful feeding and exercise will generally affect a cure. In some cases where the pigs are very small, it is well to take them away from the mother, permitting them to nurse very little. Give them castor oil in teaspoonful doses and compel them to exercise. It may be necessary to give them Choral Hydrate 10 to 15 minims two or three times a day, diluted in a teaspoonful of water. Where the pigs will not eat mashes or drink milk, give them medicine by force with a teaspoon.

AFTER TREATMENT. Feed Dr. Korinek's Stock Tonic. It contains bitter tonics which are indicated in digestive disorders.

\section{Whip Worm}

This worm is very uncommon, but is occasionally found in large intestines.

CAUSE. The eggs become imbedded in the manure, bedding, etc., and then mix with the feed and drinking water and taken into the digestive canal where they develop into matured worms. This worm is from one to three inches in length, the hind extremity of which is very thin, hence the name, "Whip Worm."

SYMPTOMS. They produce very little disturbance even though present in large quantities, except when other worms assist in irritating the lining membranes of the large intestines.

TREATMENT. Medical treatment; withhold all food from eighteen to twenty-four hours, then give one teaspoonful of gasoline thoroughly mixed with milk to every one hundred pounds of hog weight. To small hogs reduce the dose in proportion to their weight. It is advisable to follow this dose for two or three consecutive days. Give food that is easily digested and see that they have fresh water to drink. 



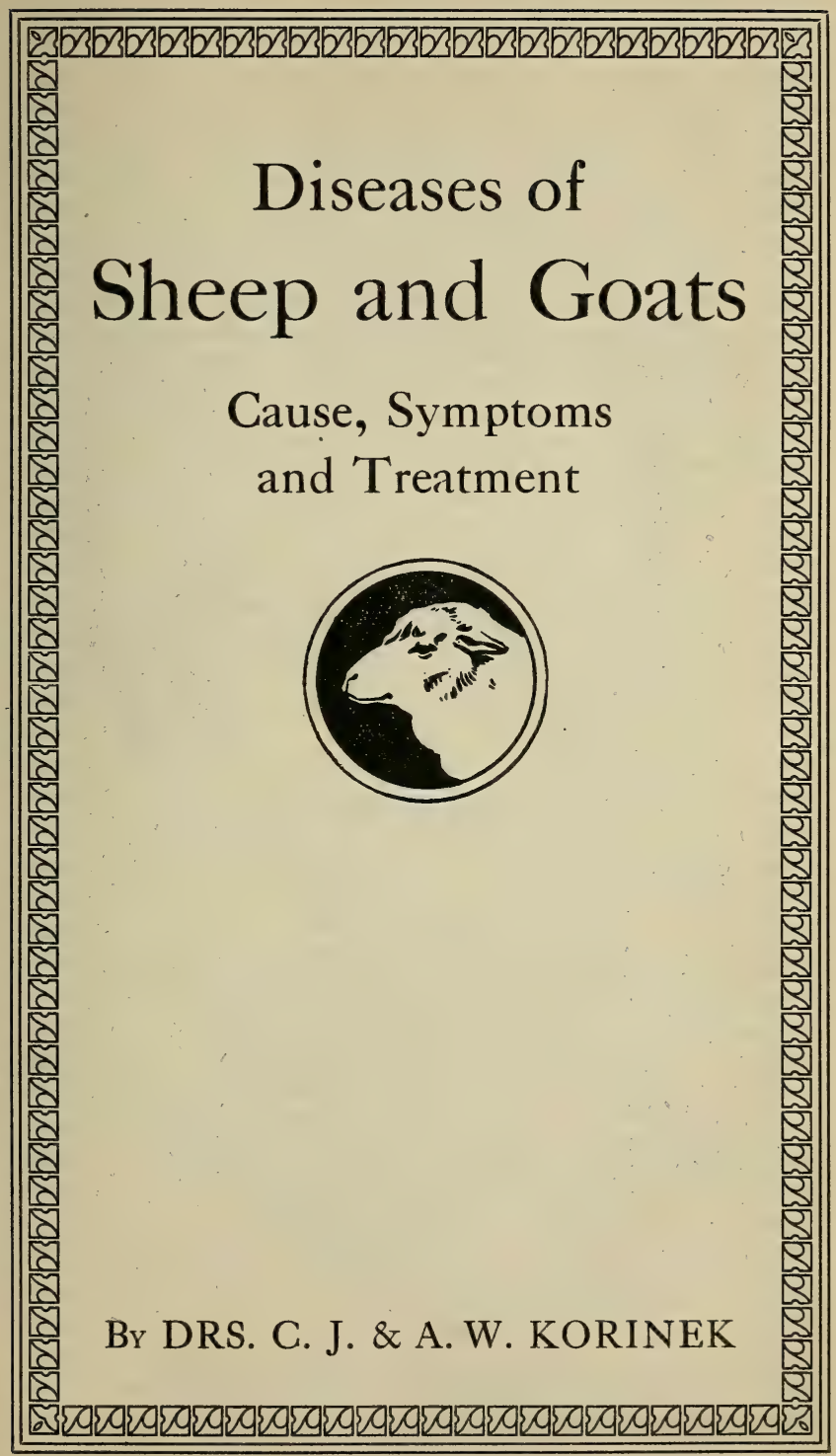




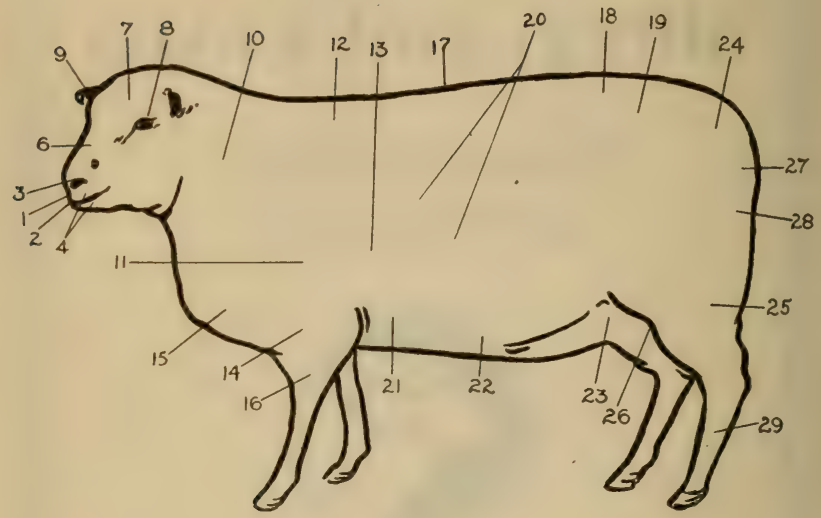

POINTS OF SHEEP

$\begin{aligned} 1 & \text { Muzzle } \\ 2 & \text { Mouth } \\ 3 & \text { Nostril } \\ 4 & \text { Lips } \\ 6 & \text { Face } \\ 7 & \text { Forehead } \\ 8 & \text { Eye } \\ 9 & \text { Ear } \\ 10 & \text { Neck } \\ 11 & \text { Shoulder Vein } \\ 12 & \text { Shoulder Top } \\ 13 & \text { Shoulder } \\ 14 & \text { Arm } \\ 15 & \text { Brisket }\end{aligned}$

1 Muzzle

2 Mouth

3 Nostril

4 Lips

6 Face

7 Forehead

8 Eye

9 Ear

10 Neck

11 Shoulder Vein

12 Shoulder Top

13 Shoulder

15 Brisket
16 Foreleg

17 Back

18 Loin

19 Hip

20 Ribs

21 Fore Flank

22 Belly

23 Hind Flank

24 Rump

25 Leg of Mutton

26 Udder or Pouch

27 Dock

28 Twist

29 Hind Legs 


\section{Black Scours (Strongylosis)}

This is a parasitic disease caused by a small thread-like worm which is pink or whitish in color and twisted in shape, measuring from one to one and a quarter inches in length. Infection is due to animals being pastured on land where the disease has previously existed or by introducing a diseased animal into the flock.

SYMPTOMS. Languor, the animal lags back from the flock, shows great stupidity, loss of flesh and excessive diarrhoea; membranes of the mouth, nose and eyes are pale, owing to the absence of blood; swelling may appear in the region of the head and neck; there is always great thirst, and the animal becomes so weak that at last it falls and is unable to rise. The disease causes great loss to sheep owners and every one owning sheep or goats should be familiar with the characteristic symptoms of the disease and the treatment necessary to control it when it appears.

POST MORTEM. The muscles after death are found to be flabby, bloodless and easily torn. The blood is watery and very pale in color. The fourth stomach (Abomasum) and the small intestines contains a chocolate-colored fluid in which are swimming myriads of small worms. Unless one is familiar with their appearance, these worms can only be seen with the aid of a magnifying glass.

TREATMENT. Give Dr. Korinek's Calf Scour and Cholera Remedy (one tablespoonful twice daily) until the desired results have been obtained. Change pastures, separating the heaithy sheep from those diseased. Feed rich food and avoid giving too much water.

\section{Bloating}

The digestive organs of the sheep are similar in construction to those of the cow, and, like cattle, rhey are subject to bloating.

CAUSE. Usually due to eating green clover or altalfa wet with dew or rain. It may, however, be due to indigestion arising from some other cause.

SYMPTOMS. The left flank becomes abnormally large; this condition is accompanied by colicky pains, difficult breathing, and a flow of stringy saliva from the mouth; the animal shows signs of great uneasiness. Unless promptly and properly treated, there is danger of rupture of the diaphragm (the muscular wall between the chest and the abdomen) or the stomach may become so distended with gas as to afford no room for the lungs to expand, and the animal suffocates.

TREATMENT. Give Dr. Korinek's Anti-Cow Bloat Capsules, as directed, and in severe cases it is advisable to put a gag in the mouth. This treatment will usually cure the most stubborn case if given promptly. 


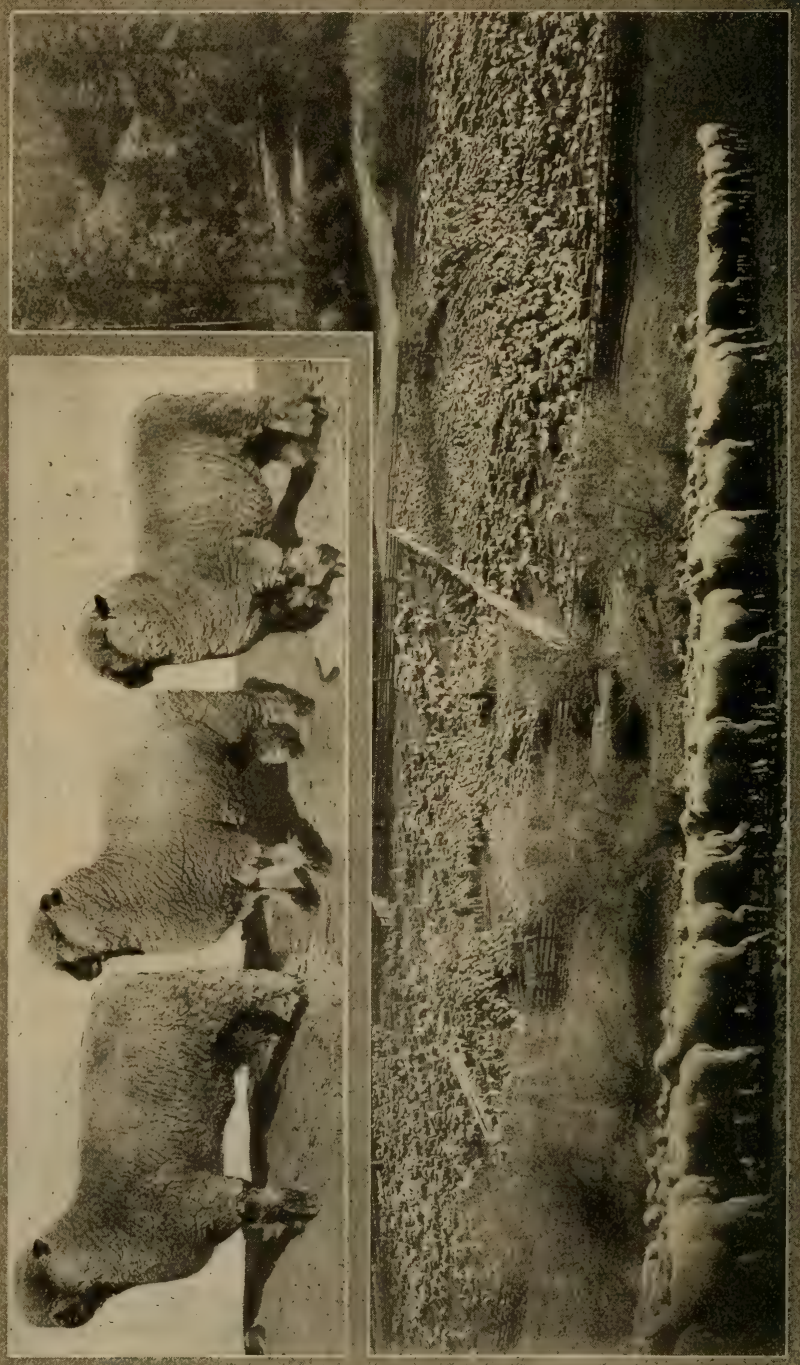

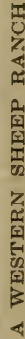




\section{Catarrh (Bad Cold)}

Sheep and goats are particularly subject to colds and catarrh.

CAUSE. Usually due to a sudden chill; often caused by shearing early in the spring before warm weather sets in, or by dipping in cold weather, etc.

SYMPTOMS. Watery discharge from the nose and eyes, coughing, sometimes loss of appetite, quick breathing, and general unthriftiness.

TREATMENT. Avoid shearing and dipping when the weather is cold, and see that the animals are warmly housed. Give one tablespoonful of Dr. Korinek's Distemper Remedy, three times daily. When the breathing is very difficult, inhalations of steam from hot water and turpentine is beneficial (one tablespoonful of turpentine in one gallon of boiling water). Sheep are naturally delicate and require special care and attention when sick or injured.

\section{Contagious Foot Rot}

This form of Foot Rot is due to a germ and is usually communicated to the entire flock.

SYMPTOMS. The first sign is a slight lameness, and when the foot is examined it is found to be red and sore between the toes, all four feet may be affected. As the disease progresses sores and ulcers form around the top of the foot and discharge a cheesy substance very fetid in odor. Animals affected with this disease suffer great pain and will lie down most of the time.

TREATMENT. Remove the cause, whatever it may be, if due to rocky pastures, remove to soft pastures and if the sheep are too numerous to treat separately, build a trough, fill it with a solution of Dr. Korinek's Sheep Dip (one part dip to fifty parts water), drive the sheep through this solution once daily until the feet are entirely well. If possible, use Dr. Korinek's Hoof Remedy; it will give quicker results than dip.

\section{Diarrhoea and Dysentery}

Unusual in old sheep and goats, but quite common to lambs and kids.

CAUSE. The cause may be of an infectious nature or it may be the result of improper feeding.

SYMPTOMS. Watery passages from the bowels and great weakness; the animals soon become very poor and unthrifty looking. The passages finally become tinged with blood, the animal refuses food and if not properly treated, will die.

TREATMENT. Give Dr. Korinek's Calf Scour and Cholera Remedy as directed and see that the animal is well fed and cared for. 


\section{Foot Rot}

CAUSE. Ordinary Foot Rot may be caused by rocky pastures. The feet at first get sore and finally become infected with different germs in the interdigital spaces or between the toes, which causes great pain and lameness.

SYMPTOMS. Lameness. Sheep may become so lame that they are unable to walk and hobble along on their knees. The hoofs become very long and dry and oftentimes crack or break. This form of foot rot differs from contagious foot rot in that it is not infectious.

TREATMENT. Remove the cause, whatever it may be, if due to rocky pastures, remove to soft pastures, and if the sheep are too numerous to treat individually build a trough and fill it with a solution of Dr. Korinek's Sheep Dip (one part dip to fifty parts water), drive the sheep through this solution once daily until the feet are entirely well. Dr. Korinek's Hoof Remedy gives quick results and should be used if possible.

\section{Grubs in the Head}

CAUSE. Due to a fly (Oestrus Ovis) which attacks the sheep during the summer months, depositing its eggs in the nostrils of the animals. These grubs make their way up the nasal cavities into the sinuses between the eyes and attach themselves to the mucous membrane.

SYMPTOMS. When the sheep are attacked by these flies, they run, strike at the nose with the feet, rub the nose on the ground, and raise the head in the air. There is a discharge from the nostrils, loss of appetite, great weakness and frequent attacks of coughing and sneezing. Animals become so weak they reel when they walk, and when no longer able to stand, they lie down and finally die.

TREATMENT. It is best to prevent the deposit of the larvae by smearing the sheep's nose with tar. It is a good plan to make troughs to contain salt, boring holes in the sides of the trough large enough to admit the nose of the sheep and keep the edge of the holes well smeared with tar. The only way to remove the worms is by trephining; this, however, requires a considerable amount of skill and a special instrument.

\section{Indigestion (Impaction)}

CAUSE. As a result of feeding on dry pastures or eating dry foliage, etc., sheep are subject to indigestion and impaction, especially where water is scarce. 
SYMPTOMS. Any falling off in condition is very noticeable among sheep, as the slightest indisposition is followed by every sign of unthriftiness. One of the first symptoms of indigestion is loss of appetite, which is always accompanied by loss of appetite; irregularities of the bowels, constipation; the animal stands motionless and makes no attempt to eat or drink.

TREATMENT. If the animal is constipated give one of Dr. Korinek's Physic Capsules and rectal injections of warm soapy water. When the appetite returns feed laxative food. Remove to better pastures or feed moistened food and plenty of pure water.

\section{Lice}

Different forms of lice affect different animals, but the only one that seems to be prevalent in sheep and goats is called Hematopinus Stenopsis.

SYMPTOMS. Scratching on rubbing posts, fences, etc., skin becomes reddened, the wool is stringy and matted; these lice seem to cause more trouble in spring and winter.

TREATMENT. The treatment depends on the atmospheric conditions. If the weather is suitable they should be dipped with Dr. Korinek's Sheep Dip. If affected in cold weather use Dr. Korinek's Lice Powder.

\section{Lip and Leg Disease (Lip and Leg Ulceration)}

CAUSE. Due to the germ Bacillus Necrophorus, cuts, abrasions, and bruises afford channels for infection.

SYMPTOMS. The disease usually affects the parts which are free from hair, as the lips, face, chin, nose, gums and soft palate, and sometimes, though less frequently, the legs and feet; the external genitals of both the ram and ewe are commonly affected. The parts are very much inflamed and covered with small pimples which spread and unite, forming at last one large scab of a grayish color. When this scab is removed the skin beneath is found to be ulcerated and tumid, swelling two or three times the normal size. The appetite remains good but the condition of the lips is usually such that the animal is unable to eat.

TREATMENT. Prevention is of course the best treatment. Keep the sheep free from infection, separating those diseased from the rest of the flock. When new animals are to be introduced into 
the herd, they should first be quarantined for two or three weeks to make sure that they carry no infection. Corrals, stables, walls, mangers, and rubbing-posts should be thoroughly disinfected, as the germs have a marvelous vitality and thrive where least expected. For this purpose use Dr. Korinek's Dip, one part dip to seventy parts water.

MEDICINAL TREATMENT. Very good results are obtained by removing the scab with a rather blunt stick (avoid using a sharp instrument) and applying the following dressing to the parts: Five parts Dr. Korinek's Dip, ten parts Sublimed Sulphur, one hundred parts mutton or tallow. Apply this dressing with a swab (a cloth wrapped around the end of a stick) three or four times a week until well.

\section{Leech or Liver Fluke (Distomiasis)}

CAUSE. Small parasite which invades the bile ducts of the liver. This disease is most prevalent in the low bottom-lands, and quite uncommon on hill pastures.

SYMPTOMS. The sheep become thin and weak, the membranes of the nose and eyes are pale, bloodless and yellowish in color; loss of appetite, and usually swelling of the neck and jaws. The droppings contain these parasites which are small, flat and leaf-like, and about one and one-fourth inches long and one-third of an inch wide. Pregnant ewes when affected with this disease will abort.

TREATMENT. Give calomel, two to ten grains according to size and age. Change to uninfested pastures and rich food are essential.

\section{Mange in Sheep and Goats}

There are three different kinds of mange but the one affecting sheep and which is the most common in the United States is called Psoroptes Communis. This variety of mange is plentifully found in the Southwestern States.

SYMPTOMS. Sheep affected with scab parasite are found to be restless, biting themselves. Bunches of wool become loosened and drop off. If the skin is closely examined the parasite may be seen with the naked eye or by scraping the irritated part of the skin and putting the scrapings in a bottle in a warm place or in the sunshine, when the parasites may be seen crawling on the walls of the glass. The back and rump are the parts affected and denuded of wool. If animals in this condition are neglected they become very poor and finally die. Mange is not only very destructive to the animal but spoils the wool in quality as well as to greatly reduce the supply. 
TREATMENT. Dipping is the only treatment that has met with success. Most sheep men have large vats to dip their sheep in. The sheep should be forced to swim for at least a short distance in a solution of Dr. Korinek's Sheep Dip, one part dip to seventy parts of water. This same treatment should be repeated in ten days. Also disinfect the sheep barns or place where the sheep are housed, with the above solution, or if possible change to pastures where scab is not present.

\section{Sheep Ticks (Louse Fly)}

These parasites are bloodsuckers and affect mostly the longwool sheep.

SYMPTOMS. Sheep affected with this parasite rub until the skin becomes red. It is more noticeable after shearing. The ticks leave the sheep that have been sheared and infest the lamb.

TREATMENT. The whole flock should be dipped with Dr. Korinek's Sheep Dip, one part dip to seventy parts water. Repeat the treatment about ten days later and place the sheep in new quarters. The barns or places where the sheep are housed should be disinfected with Dr. Korinek's Sheep Dip.

\section{Sheep Pox (Sheep Variola)}

CALSE. Due to a germ. This disease is not common in the United States.

SYMPTOMS. Chills, fever, temperature as high as $108^{\circ} \mathrm{F}$., debility, loss of appetite, discharge from the eyes, eyelids swollen, discharge from the nose and offensive breath. After a day or two a rash appears on the skin followed by vesicles or small elevations which finally burst, leaving a small round patch. The disease attacks such places as the face, inner part of the thigh, under surface of the tail, lower chest and belly where the wool is absent.

TREATMENT. Cleanliness; bathe the affected parts with a solution of acetate of lead and give laxative food. If the flies are bad, keep the animals in the barn through the day and turn them out at night. If the number of animals affected does not admit of treating them individually, it may be necessary to dip them.

\section{Verminous Bronchitis}

Inflammation of the mucous membrane of the lungs and bronchial tubes, due to small worms.

CAUSE. Small thread-like worm, about three inches long and white in color. Both sheep and goats are affected by this disease. 
SYMPTOMS. Loss of appetite, the animals become poor and unthrifty in appearance. There is usually a cough and accelerated breathing due to the plugging of the air passages with worms and mucus. The cough becomes more painful as the disease advances and the sheep may die of convulsions or suffocation. In some instances the worms are coughed up.

TREATMENT. Steaming with medicated vapor is the only treatment that has met with success. Perhaps the nost effective way of giving inhalations is by placing the sheep in a tightly-closed room with a bucket of boiling water containing a tablespoonful of oil of turpentine or oil of eucalyptus. This treatment should be repeated once a week for three or four weeks. The sheep should be left in the room until the coughing is well marked; a man must use his own judgment as to the amount they can stand. 



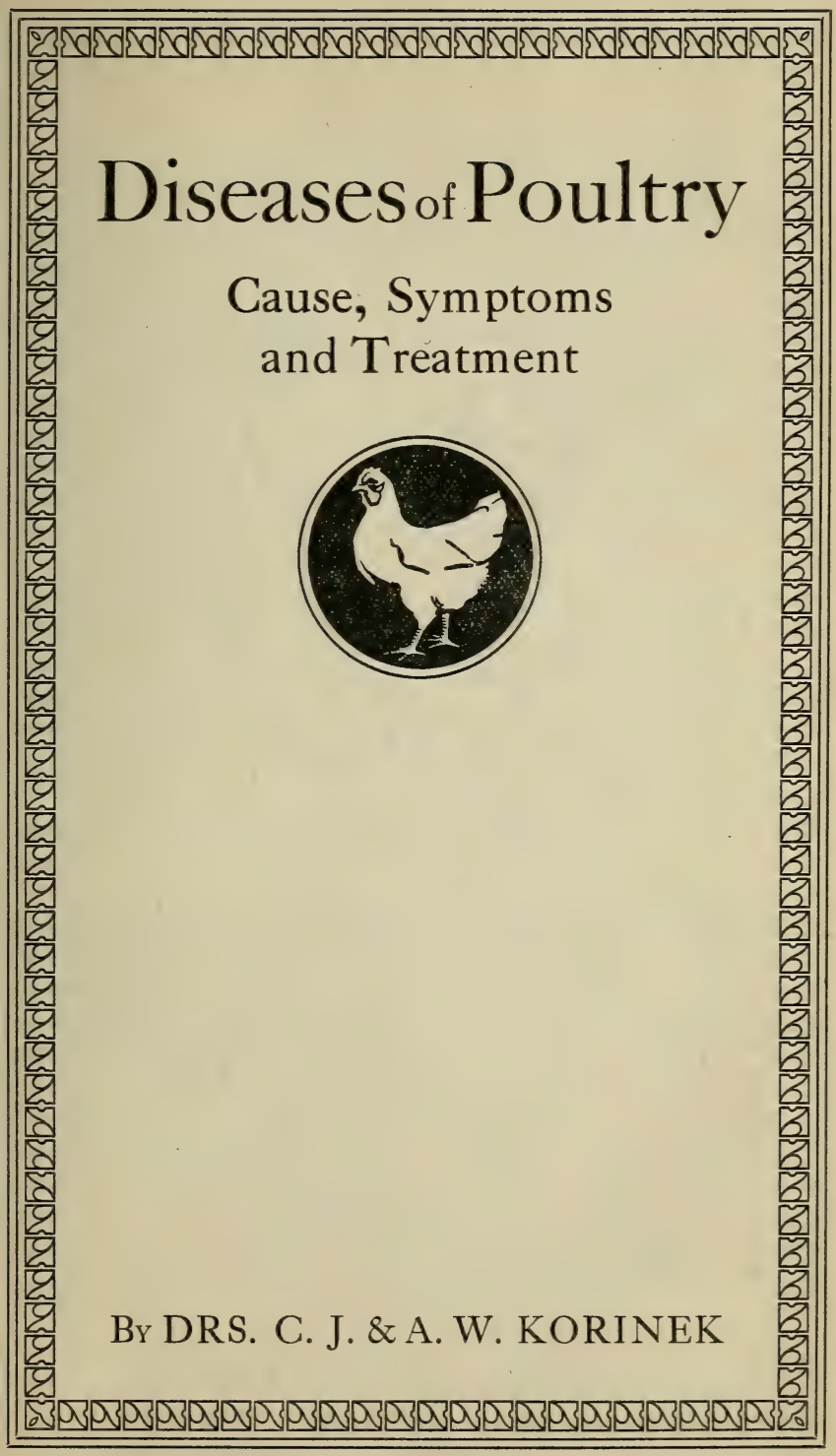




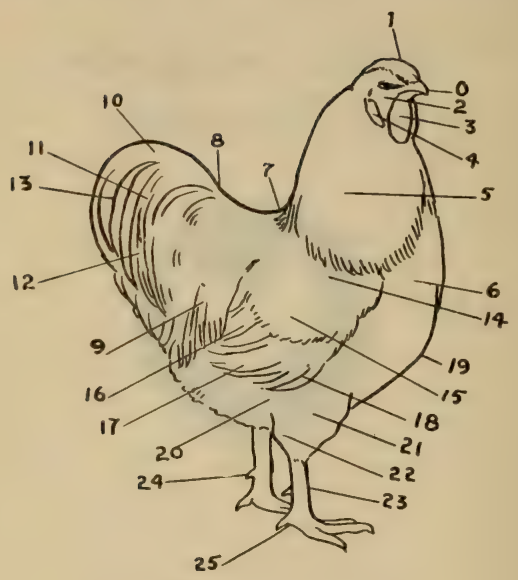

POINTS OF THE FOWL

0 Beak

1 Comb

2 Face

3 Wattles

4 Ear Lobe

5 Hackle

6 Breast

7 Back

8 Saddle

9 Saddle Feathers

10 Sickles

11 Lesser Sickles

12 Tail Coverts

13 Main Tail Feathers
14 Wing Bow

15 Wing Coverts, forming Wing Bar

16 Secondaries, Wing Bar

17 Primaries or Flight Feathers

18 Flight Coverts

19 Point of Breast Bone

20 Fluff

21 Thigh

22 Knee Joint

23 Shank

24 Spur

25 Toes, or Claws 


\section{Air Sac Mite}

CAUSE. Produced by a parasite called Cyloditis Nudus, which bears a close resemblance to the parasite causing mange or scabies in the domesticated animal. Owing to the peculiar construction of their breathing organs, fowls are more susceptible to parasites than are animals. In addition to affecting the lungs the Air Sac Mite may extend its operations to the intestines, kidneys, liver and bones.

SYMPTOMS. Unthriftiness is first noticed, but after the parasites become numerous the fowl shows signs of difficult breathing, perhaps terminating in bronchial pneumonia. In some cases death occurs without apparent cause. The bird will be inactive, become separated from the rest of the flock, comb pale, head drawn close to the body, wings hang pendulous, lose flesh, breathing becomes hard, coughing, sneezing and a rattling from the mucous in the wind pipe is heard. Death is produced from suffocation.

TREATMENT. Separate the sick from the healthy fowls. Disinfect coops and runways of both sick and healthy birds with Dr. Korinek's Sheep Dip and Disinfectant. Dr. Korinek's Poultry Tonic contains ingredients that are strongly recommended in this particular disease. It is advisable to fumigate the fowls in their coops with steam from hot water and pine tar. This may be done by placing the water and pine tar in a pan and then inserting a hot stone or brick in the solution. This is perhaps the simplest form of fumigation. I am positive that poultry would be immune from this form of parasite if Dr. Korinek's Roup and Cholera Remedy was added to their drinking water occasionally.

\section{Apoplexy (Haemorrhage of the Brain)}

Due to the rupture of a blood vessel of the brain and pressure from the escaping blood.

CAUSE. Mechanical injuries, straining when laying eggs (hens are frequently found dead on the nest from this cause), overfeeding, stimulating food, etc., all tend to produce apoplexy.

SYMPTOMS. Appear very suddenly, bird is seen to walk unsteadily, falls, or perhaps is found dead.

TREATMENT. In mild attacks apply cold water or ice to fowl's head until thoroughly cooled. Give one-half grain of calomel, feed soft food, compel the bird to exercise. Owing to the loss of blood, a tonic will be necessary. Dr. Korinek's Poultry Tonic has medicinal properties that purify and build up the blood.

\section{Beak and Throat Obstruction}

CAUSE. Lodgment in the beak or food canal of a foreign substance, such as a kernel of corn, sunflower seed, bone, etc. 
SYMPTOMS. Fowl jerks its head suddenly, and frequently attempts to swallow. If a close examination is made the foreign body can be felt from the outside.

TREATMENT. If obstruction can be seen when fowl's mouth is opened it can easily be removed with a tooth pick, clean sharp wire or a long needle. If not to be seen, give small quantities of olive oil or sweet oil and carefully manipulate the throat with an upward movement until obstruction can be seen and removed as above described.

All weak fowls should be given Dr. Korinek's Poultry Tonic as directed on the package.

\section{Body Lice} tact.

CAUSE. Unsanitary conditions. Communicated by direct con-

SYMPTOMS. Young chicks become emaciated and die quickly. Older birds withstand the parasite much longer, but in time show signs of uneasiness by dusting themselves frequently. The comb and wattles become pale and bloodless, the feathers rough, dry and brittle. The birds grow weak, poor and eventually die.

TREATMENT. Dust the birds with Dr. Korinek's Lice Powder, also sprinkle it freely in the dust beds.

\section{Baldness (Favus)}

CAUSE. Due to fungi similar to that which attacks cats, rabbits and mice.

SYMPTOMS. The first noticeable sign is the whitish appearance of the comb, due to gray spots about the size of a pin-head. As the disease progresses, this condition spreads to other parts of the body; the feathers look rough and dry and break easily. The fowl grows weaker, refuses to eat and if not properly treated dies.

TREATMENT. Remove the scabs by separating the feathers and using a brush, and apply Dr. Korinek's Dip (one teaspoonful to a pint of water). Repeat this treatment every two days; great care must be taken after each application that the fowls do not chill. This treatment has proven very successful.

\section{Black Head (Infectious Entero-hepatitis of Turkeys)}

CAUSE. Due to a protozoa taken into the system with the food or drinking water. This parasite enters the caeca which become inflamed and discolored and the liver is enlarged and studded with yellowish spots about the size of a pea. 
SYMPTOMS. Although this disease is termed Black Head, the discoloration of the head is not necessarily present in all cases; neither is this condition confined to this particular disease. One of the first symptoms is loss of appetite, followed in most cases by diarrhoea. The fowl becomes weak and loses weight rapidly. Examination of the liver after death will determine whether or not death has been caused by infectious Entero-hepatitis. The dead birds should be burned to prevent the spread of the disease.

TREATMENT. Prevention is one of the most important factors, as the disease is very contagious and the protozoa once implanted in the turkey runs is hard to eradicate. Provide clean, light, wellventilated coops and clean, wholesome food, to which add Dr. Korinek's Poultry Tonic as directed on package. The drinking water should contain Dr. Korinek's Roup and Cholera Remedy; allow no other water as it may contain disease germs. Dr. Korinek's Roup and Cholera Remedy is a powverful and guaranteed germ killer.

\section{Bumblefoot, Corns, Deep Bruises, and Abscesses}

CAUSE. Sharp-edged or narrow perches which bruise the feet or where the perches are high, heavy fowls often injure their feet by alighting on stones or other hard objects.

SYMPTOMS. The bird limps or hobbles about, moving with great difficulty. Examination will show the foot to be hot and tender to the touch.

TREATMENT. Wash with clean warm water and apply a hot flaxseed poultice; when a soft spot appears, lance with a clean sharp knife. If the abscess or bruise is already open, treat antiseptically by washing in a solution of Dr. Korinek's Dip and Disinfectant (one teaspoonful to a pint of water). The foot should be bandaged to keep out the dirt.

\section{Bronchitis}

CAUSE. Exposure to dampness, cold draughts of air, inhaling irritating gases, vapors or dust. The fowls should be carefully examined, as bronchitis is occasionally caused by the presence of gape worms.

SYMPTOMS. Loss of appetite, the bird moves about slowly, breathing with difficulty and making a sort of whistling sound accompanied by a cough. As the disease progresses there will be a peculiar bubbling sound when breathing, due to an excessive accumulation of mucous in the wind pipe. At this stage of the disease the bird becomes very weak and if not properly treated and cared for will rapidly lose strength, the feathers will become rough, head and wings droop, and the bird dies. 
TREATMENT. This disease is most satisfactorily treated by placing the sick birds in warm, dry, well-ventilated quarters; exclude all draughts of air; feed stale bread, middlings, etc., together with Dr. Korinek's Poultry Tonic moistened with milk. Compel birds to drink water containing Dr. Korinek's Roup and Cholera Remedy; it has a soothing effect on the organs of breathing.

\section{Catarrh-Roup}

CAUSE. Exposure; poorly constructed coops, which admits rain or drafts. Weak birds are very susceptible to catarrh.

SYMPTOMS. The bird is dull, moves about slowly, coughing or sneezing; appetite is poor, the mucous membrane of the air passage becomes inflamed and the breathing difficult, especially through the nose. The discharge from the nostrils at first watery, becomes mucous-like and finally thick and sticky, closing the nose, causing the bird to breathe wholly through the mouth with a wheezing sound.

TREATMENT. The cause of catarrh shows the necessity of clean and comfortable quarters for the fowls. Keep the birds strong and vigorous by feeding Dr. Korinek's Poultry Tonic, and allow no other water but that containing Roup Remedy.

\section{Chicken Pox; Sore Head; Warts}

CAUSE. These diseases are due to low forms of parasites or fungi and occur most frequently in wet weather, especially if the coops are leaky and allow the rain to fall on the droppings, causing mold or fungi. Poor ventilation and lack of light also promotes the growth of fungi.

SYMPTOMS. The disease is usually confined to the head and affects principally young chickens, pigeons and turkeys, but rarely ducks and geese. The infection appears in the form of yellowish warts or nodules about the nose, eyelids, comb, wattles, under the wings, or any unfeathered places. These warts vary in size from that of a pin-head to the size of a pea and they discharge a fluid which at first is thin and watery but as the disease progresses it becomes thick and sticky, yellow in color and fetid in smell. At this stage the appetite is poor, the feathers appear rough, and where the eyelids are affected, as in most cases, the bird cannot see, fails to eat, becomes emaciated, loses weight and strength rapidly, and if not properly treated, dies.

TREATMENT. The disease is very contagious; therefore the coops and runs should be cleaned and disinfected with Dr. Korinek's Sheep Dip and Disinfectant (one part dip to fifty parts water) or a solution of one part carbolic acid to ten parts water. The drinking water should contain Dr. Korinek's Roup and Cholera 


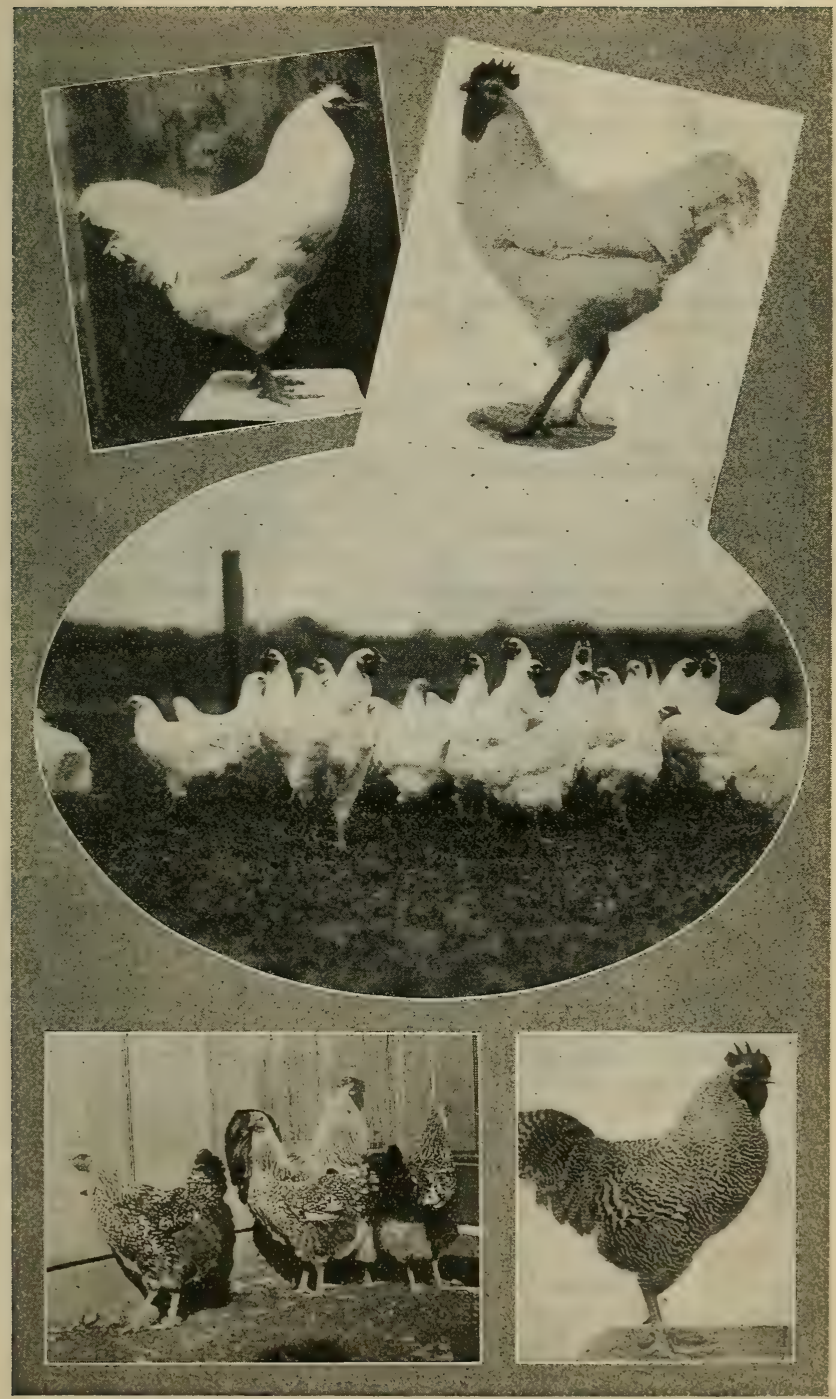


Remedy; allow no other water. Wash nodules or warts about the head with dip, one teaspoonful to a pint of water (this is not injurious to the eyes). Feed Dr. Korinek's Poultry Tonic frequently, as it contains medicinal values for the treatment of this particular disease.

\section{Congestion of the Liver}

CAUSE. Lack of exercise, overfeeding, tainted or moldy food, or poor blood.

SYMPTOMS. Birds suffering from this disease seldom show signs of sickness and it is well to dissect the fowl after death to ascertain the exact cause. If death is caused by congestion of the liver the organ will be greatly enlarged and easily torn.

TREATMENT. If the fowls are fat and sluggish, compel them to exercise by driving them about; also give about fifteen or twenty grains of Epsom Salts to ,each bird. Follow with Dr. Korinek's Poultry Tonic, which purifies the blood and relieves congestion by stimulating the circulation.

\section{Congestion of the Lungs (Pulmonary Congestion)}

CAUSE. Exposure; the bird chills, causing contraction of the blood-vessels near the surface of the body, thereby forcing a large quantity of blood to the internal organs; the small blood-vessels in the lungs become distended with blood and rupture.

SYMPTOMS. Rapid and difficult breathing; the bird appears stupid and sleepy and does not care to move about; appetite poor, wings drooping, plumage ruffled, a thick mucus, colored with blood escapes from the mouth, comb and wattles show a dark-red color from lack of oxygen in the blood. This disease is of very short duration, the bird dying within a few hours. It is very common among young chicks and turkeys that are permitted to run out in the early spring rains.

TREATMENT. Medical treatment is impracticable as the progress of the disease is so rapid that the bird dies shortly after the first symptoms appear. Sanitary surroundings, plenty of light, pure air (no drafts) and exercise are essential. Do not allow the birds to stand out in the cold or rain, especially during the moulting season. Keep your poultry strong and vigorous by feeding Dr. Korinek's Poultry Tonic. 


\section{Constipation (Intestinal Obstruction)}

CAUSE. Irritation of the membranes lining the intestines, caused by dry feed, glass or gravel; may also be due to parasitic worms. Obstruction may occur in any part of the intestines although the external opening is the part most frequently affected.

SYMPTOMS. Bird appears dull and stupid, walks with difficulty and attempts frequently to expel the obstructing material. The appetite is poor and the feathers rough. By examination and manipulation the obstruction may be located. Dried masses of excrement by adhering to the feathers sometimes block the outer opening of the intestines.

TREATMENT. Remove the waste matter clinging to the feathers with warm water or by clipping the feathers. If the cloaca is obstructed, give injections of sweetoil or oliveoil with small blunt syringe. Also give one or two grains of calomel and feed green food and soft mashes containing Dr. Korinek's Poultry Tonic, which, by stimulating the worm-like movement of the bowels, assists in expelling their contents and prevents constipation. Dr. Korinek's Poultry Tonic will also clear the intestines of worms.

\section{Crop Impaction, Obstruction-Paralysis, Inflammation, Catarrh}

CAUSE. Errors in feeding; birds that are not fed regularly are predisposed to any of the above conditions; may also be due to swallowing large pieces of bone, thread, nails, pins, glass and gravel.

SYMPTOMS. Loss of appetite, frequent attempts to swallow, crop greatly distended and hard on pressure; eventually the food decomposes and a liquid may escape from the mouth and nose. The bird appears dull, stupid and sleepy, comb pale, feathers rough, beak open, owing to pressure on the windpipe. If caused by swallowing sharp objects, they may penetrate the crop and skin, causing a gangrenous condition. Grain in the crop will sometimes send out sprouts of considerable length.

TREATMENT. If no sharp objects are present, give two teaspoonfuls of sweet or olive oil; this will lubricate the oesophagus and crop. Manipulate upward, forcing the food gently through the mouth, adding oil occasionally. If, however, sharp objects penetrate the crop it is best to remove them through an artificial opening. Clip the feathers from around the intended seat of operation and wash the clipped surface with Dr. Korinek's Dip and Disinfectant, one teaspoonful to a pint of water, or a carbolic solution of the same strength. The incision should not be over an inch long and should be made as high as possible in the center. After removing the contents of the crop, sew up with ordinary thread and needle and wash occasionally with one of the above antiseptic solu- 
tions. The operation is not difficult and will be successful if the crop is not too badly inflamed. After treatment consists in feeding very little food until the crop is fairly well healed; then feed soft bran mashes containing Dr. Korinek's Poultry Tonic, as directed. The drinking water should contain Dr. Korinek's Roup and Colera Remedy; it has a healing effect and relieves catarrhal conditions of the crop and intestines.

\section{Diarrhoea-Gastro-Intestinal Catarrh- Gastro Enteritis-Enteritis}

CAUSE. Inflammation of the digestive organs can be traced in every instance to the quality and quantity of food or water consumed. The food or water may contain parasites, or large quantities of mustard, pepper, or may be moldy or tainted.

SYMPTOMS. Loss of appetite, the feathers appear rough, the crop is sometimes paralyzed and distended with gas, the bird moves slowly, the droppings vary in color from a white to a yellow or a green and finally become tinged with blood; at this stage there is a rise in temperature accompanied by great thirst and signs of pain. Mild cases of simple diarrhoea if not properly treated when the first symptoms appear, will develop the same severe conditions described above.

TREATMENT. Determine the cause and remove if possible. See that the food is nutritious, the coops well ventilated, and the runs well lighted; sunshine is very beneficial. Avoid exposure, drafts and dampness. Keep Dr. Korinek's Roup and Cholera Remedy and Oat Meal in the drinking water. Also give two grains of Bismuth Subnitrate, mixed with dough and formed into a little pill, every six hours. When in addition to the above symptoms, a bloody discharge is present, give six drops of tincture Catechu every four hours. Dr. Korinek's Poultry Tonic should be mixed with warm mashes of bran or oat meal.

\section{Diphtheritic Roup (Diphtheria)}

CAUSE. Due to a specific germ. The disease is very contagious and is communicated by direct contact. Great care should be exercised therefore when showing or buying birds. Any new birds to be added to the flock should be kept in separate pens for a week or two to make sure they are in good condition.

SYMPTOMS. The first symptoms are similar to those of catarrh or cold. A clear, watery liquid escapes from the eyes and nostrils, the head is drawn in toward the body, the feathers appear rough, the breathing fast, the temperature rises from three to five degrees above normal. The bird walks about as if blind, sneezing, swallowing with difficulty, and showing signs of great weakness. 
If the mouth is opened, small white spots or elevations will be seen on the back of the tongue. There may be diarrhoea of a green or yellow color. As the disease progresses, the discharge from the nose and eyes becomes thick and stringy, obstructing the air passages and gathering in large quantities between the eyelids. The mouth, throat and tongue are very much inflamed and swollen and in most cases it is impossible for the bird to make a sound. Recovery is doubtful after the disease has reached this stage.

TREATMENT. Isolate the affected birds in clean, warm, light, well-ventilated quarters, excluding drafts. Dissolve thirty grains of Chlorate of Potash in one ounee of water and one-half ounce of Glycerine. To the average-size fowl, give one teaspoonful three or four times daily. Also keep Dr. Korinek's Roup and Cholera Remedy in the drinking water; it is an antiseptic and disinfectant. When the scum about the face becomes loosened, remove it gently; great care is necessary to prevent bleeding.

\section{Double Yolk Eggs}

Eggs are frequently found with two yolks. This condition is produced by two ovary capsules bursting at about the same time and gaining entrance together into the oviduct where they are concealed in the same shell. Double-yolked eggs are larger than normal and may injure the oviduct when expelled. When hatched they produce twins or abnormal chicks.

\section{Dropsy (Ascites)}

CAUSE. Generally due to irritating, indigestible food, causing inflammation of the membranous lining of the intestinal cavity.

SYMPTOMS. The abdomen becomes enlarged, is tender to the touch, and contains a watery fluid, the movement of which can be heard in most cases by pressure on the swollen parts. The bird appears stupid, the comb pale, and the appetite poor.

TREATMENT. Unless the bird is very valuable, it is scarcely worth while to treat the case. If treatment is desired, however, give one grain of Potassi Iodide and beef scraps containing Dr. Korinek's Poultry Tonic twice daily. This will tone the heart and kidneys and enable them to throw off the excessive fluid.

\section{Egg Bound}

\section{(Difficult Laying; Obstruction of the Oviduct)}

CAUSE. Due to the eggs being too large, the bird too fat, or to the absence of the secretions lubricating the oviduct.

SYMPTOMS. The first signs are scarcely noticeable, but soon the feathers appear rough, the bird becomes dull and moves slowly, making frequent efforts to expel the egg. 
TREATMENT. Remove the egg by injecting sweet oil and assisting the bird with gentle pressure; in some cases it is well to puncture the egg and collapse the shell. If the bird is fat, reduce by careful feeding. If the bird is of normal size the trouble is probably due to absence of the lubricating secretions of the oviduct, in which case give Dr. Korinek's Poultry Tonic, which will overcome this condition.

\section{Egg Eating}

CAUSE. Is usually due to lack of shell-building material in the food; in such case the shell of the egg is thin and easily broken and the fowl craving the lime contained in the egg shell, naturally contracts the habit.

TREATMENT. Supply ground bone and oyster shells; feed green food, such as cabbage, kale, potatoes, carrots, etc. Also feed Dr. Korinek's Poultry Tonic which contains animal charcoal and cuttle-fish bone in correct proportion.

\section{Eggs Without Shells (Soft-Shelled Eggs)}

CAUSE. Deficiency of shell material; or it is possible that fright sometimes causes premature expulsion of the egg before the shell is formed.

TREATMENT. Feed Dr. Korinek's Poultry Tonic, which contains the necessary shell material; it is best mixed in wheat bran. Also feed vegetables, such as cabbage, potatoes and carrots.

\section{Feather Pulling-Feather Eating}

CAUSE. Irritation of the skin due to lice, mites, or to lack of exercise and improper food.

TREATMENT. Feed meat, bone and vegetables containing Dr. Korinek's Poultry Tonic. Place the food where they will be compelled to scratch and work to obtain it. Also dust the fowls with Dr. Korinek's Lice Powder. It not only destroys lice and mites, but has such an obnoxious taste that it quickly discourages the featherpulling habit.

\section{Gapes-Verminous Tracheo Bronchitis}

CAUSE. A red parasitic worm, the male measuring about onefifth of an inch and the female one-half an inch in length. Fowls become infected by eating worms containing this parasite or its eggs, and by coming in contact with other birds suffering from the disease. 
SYMPTOMS. The most noticeable symptom is frequent gaping; the gape worms attach themselves by their mouths to the walls of the windpipe where they suck the blood which nourishes them; they cause irritation and inflammation of the windpipe, bronchial tubes and lungs; breathing becomes difficult and the bird loses strength rapidly; the windpipe eventually becomes totally obstructed and the bird dies from suffocation and exhaustion. Young, weak chickens are more susceptible to this disease than strong ones.

TREATMENT. Separate the sick birds from the healthy ones; clean and disinfect the coops and runs; burn all manure; remove the worms from the windpipe by the use of a feather from which the vane has been stripped, leaving only a small brush at the end. Dip the feather into oil of turpentine or coal-oil, removing the surplus liquid by drawing between the fingers; insert into the windpipe (trachea) of the bird and by turning dislodge the worms. Give Dr. Korinek's Roup and Cholery Remedy; it is a powerful antiseptic and will destroy the eggs of the worm, which are generally numerous in the intestines. Dr. Korinek's Sheep Dip is an excellent disinfectant for poultry runs and coops.

\section{Head Lice}

CAUSE. Result of unsanitary conditions and lack of care. Communicated by direct contact with infected birds, or by infected coops or brooders.

SYMPTOMS. The head soon becomes bare of feathers, and also sore by being constantly scratched with the feet. If not properly treated the chicks weaken and die.

TREATMENT. An ointment made of one part sulphur and four parts lard, well mixed and applied two or three times will effect a cure.

\section{How to Feed Young Poultry}

Withhold all food for at least eighteen hours; then feed stale bread moistened with boiled milk every three hours. When they are three or four days old, feed rolled oats, ground corn moistened with pure water, finely chopped meat and boiled vegetables. Feed them often and you will be well repaid by their rapid growth, strength, and the low death-rate. After they reach the age of one week or ten days, watch them closely and regulate their feed to their apparent needs. Dr. Korinek's Poultry Tonic contains animal charcoal, cuttle-fish bone, tonics and antiseptics, and added to the teed, aids digestion and builds bone and muscle. 


\section{Incomplete Egg (Abortion)}

CAUSE. Irritation of the oviduct; improper secretion of albumen or internal egg-producing material.

TREATMENT. Careful feeding will overcome this condition. Warm wheat bran mashes containing Dr. Korinek's Poultry Tonic will allay the irritation of the oviduct and stimulate the secretion of albumen.

\section{Liver Congestion (Jaundice)}

CAUSE. Obstruction of the bile duct, due to rich, nitrogenous food and insufficient exercise.

SYMPTOMS. Disease is not easily detected. The yellow color of the wattles and comb is the first symptom; the appetite is variable, the feathers appear rough and dry, the head is retracted and the bird finally dies owing to the absorption of bile in the blood.

TREATMENT. Change of food, preferably a vegetable diet. Give one grain of calomel. Feed regularly wheat bran containing Dr. Korinek's Poultry Tonic, which is particularly effective in cases of sluggish liver.

\section{Mange (Scabies of the Body)}

CAUSE. Due to a parasite that resembles the mite.

SYMPTOMS. When the affected bird is closely examined, large quantities of scales or scabs are found in the soft feathers. The appetite is poor; the bird walks slowly about showing signs of uneasiness. If the disease is allowed to run its course, the bird grows weak and eventually dies. The disease is easily transmitted, from one bird to another and should be treated without delay.

TREATMENT. Disinfect roosts, coops, and pens with Dr. Korinek's Dip, one part dip to seventy parts hot water. Dip the birds in solution of the same strength and place them in a warm coop until thoroughly dry.

\section{Pip (Inflammation of the Mouth)}

CAUSE. Irritation, injuries or micro-organisms. It is sometimes caused by nothing more than a dry condition of the mucous membrane due to the bird breathing through the mouth when suffering from respiratory diseases.

SYMPTOMS. Dryness of the mucous membrane of the mouth; especially the part covering the tongue, which becomes hard and ragged, forming rough edges along its sides. These dried portions become loose and partially detached from the tongue, interfering with its movements and causing more or less pain and annoyance. 
TREATMENT. Do not forcibly detach these pieces but assist nature to remove them; this can be accomplished by mixing glycerine and water in equal parts and dropping into the mouth with an ordinary dropper. Always keep Dr. Korinek's Roup and Cholera Remedy in their drinking water; it will prevent the entrance of parasites into the blood when the bird's mouth or tongue is sore or irritated. When young poultry is out of condition, drowsy, poor or weak, give Dr. Korinek's Poultry Tonic and note the difference in appearance and growth.

\section{Red Mite}

CAUŚE. They grow spontaneously in favorablc surroundings, as the interior of poultry houses and brooders containing numerous cracks and crevices.

SYMPTOMS. This mite is a blood-sucker, irritates the skir and sometimes causes sores to form on the body of the chick. The birds grow stupid and weak and die rapidly if not properly treated. Older fowls withstand the irritation of mites much longer but do not thrive or lay regularly, and will finally die if the insects become too numerous. The insect may be transmitted to horses, cattle, and even to man.

TREATMENT. Dust the affected fowl with Dr. Korinek's Lice Powder. Paint or spray the interior of coops and brooders, being careful that the paint reaches the bottom of all cracks and crevices. Also paint the interior of the nests.

\section{Rheumatism (Leg Weakness, Gout, Paralysis)}

CAUSE. Damp coops and pens, lack of ventilation and improper food.

SYMPTOMS. Fowl refuses to stand or walk, and on examination the legs are found to be swollen and painful, especially about the joints. In some cases suppuration of the joints takes place and they become open running sores. The bone finally becomes diseased and the fowl dies.

TREATMENT. Preventative measures are the first to be considered. See that the pens are dry and the coops clean, dry and well-ventilated, but avoid drafts. Feed vegetables and also wheat bran mashes containing Dr. Korinek's Poultry Tonic. When the legs are swollen and sore, apply camphorated ointment once or twice daily. 


\section{Scaly Leg (Scabies)}

CAUSE. Due to a mite that burrows under the scales of the leg.

SYMPTOMS. White, scaly-looking scabs form about the upper part of the foot. The feet and legs become swollen and painful as the disease progresses and if not checked will result in lameness, inflammation of the joints, and the toes may slough off. Great care is necessary as the disease is very easily transmitted from one bird to another.

TREATMENT. Use boiling water or some strong disinfectant on the perches. Wash the feet and legs with warm water and soft soap; dry well and apply Carbolic Ointment. Repeat the above treatment every other day for a week.

\section{Sore Mouth-Apthae-Thrush}

\section{CAUSE: A vegetable parasite called Oidium Albicans.}

SYMPTOMS. Inflammation of the mucous membrane lining the mouth, throat, gullet, and crop, which finally terminates in white ulcerations. Other symptoms are swelling of the head, poor appetite and a rapid loss in weight and strength.

TREATMENT. Isolate all sick birds from the healthy ones and give as much sunlight as possible; feed good nourishing food, such as warm oatmeal mashes, kale, potatoes and green food. Keep Dr. Korinek's Roup and Cholera Remedy in the drinking water; it saves the time and trouble of washing each individual mouth. We guarantee to prevent Aptha and to cure it in its first stages. A good disinfectant must be used to prevent the disease from spreading; Dr. Korinek's Sheep Dip and Disinfectant is warranted to give satisfaction.

\section{Tuberculosis}

CALSE. This dreaded disease is caused by the Bacillus of Tuberculosis. Damp, ill-ventilated and poorly-lighted coops are favorable to the development of the disease.

SYMPTOMS. Except in advanced stages, this disease is not - easily detected as it affects various organs, and considerable experience in post-mortems and a skillful use of the microscope is required to successfully diagnose a case.

TREATMENT. Preventative measures should be taken as the disease is incurable. Do not expose the fowls to cold, wet weather. See that the coops are well ventilated and lighted and feed no contaminated food. 


\section{Vent Gleet}

CAUSE. Constipation is perhaps the most common cause, the hard droppings causing irritation of the vent which is followed by inflammation and suppuration of the lining membranes of th rectum and oviduct.

SYMPTOMS. Frequent straining due to irritation. As the disease progresses, a pus-like discharge is noticed. The disease may extend into the rectum or oviduct. The bird appears stupid, the plumage rough, the comb pale, and if not properly treated, dies a lingering death.

TREATMENT. Preventative treatment is the best. Allow green food occasionally and warm bran mashes, thus preventing constipation. When the bird strains frequently and a discharge is present, the following solution should be injected: Sugar of Lead, two drams; Zinc Sulphate, one dram; two quarts of water. Inject about onehalf ounce with a syringe twice daily.

\section{White Diarrhoea or Fowl Cholera}

CAUSE. Germ (Bacilli of Fowl Cholera) gaining entrance to the body through the bowels, lungs or wounds of the skin. Death results from toxic material produced while the germs are multiplying.

SYMPTOMS. All poultry, cage or wild birds are subject to this disease. The first symptom is loss of appetite; diarrhoea is present and the discharge is almost white in color and tinged with transparent mucus. The affected bird becomes separated from the flock, seems weak and stupid and appears to be asleep; feathers are rough, is generally full, owing to improper digestion. The comb is pale and bloodless, the temperature rises from three to five degrees above normal and the bird loses weight rapidly; it may die with convulsions and cries or without a sound or struggle.

TREATMENT. Give Dr. Korinek's Roup and Cholera Remedy in the drinking water as directed on package. Also feed Dr. Korinek's Poultry Tonic; it contains stimulants and antiseptics that assist nature in overcoming the disease. In severe cases of diarrhoea give Bismuth Salicylate, one grain three times daily in the feed or made into a pill with dough and given by hand. Vaccination of fowls is discouraged as it is very costly and difficult of accomplishment and different birds require different sized doses. 


\section{Worms}

CAUSE. Few fowls are entirely free from worms. The soil over which the chicks are permitted to run may be infected, or the food may contain the eggs or embryos of worms.

SYMPTOMS. The presence of worms in fowls may not be at once detected, since only a close observer would notice them in the droppings. If the birds eat well but remain poor, and the feathers appear rough and the comb and wattles pale, there is reason to suspect the existence of worms.

TREATMENT. Preventative treatment is the best. Sprinkle the pens and coops regularly with Dr. Korinek's Lice Powder, also mix Dr. Korinek's Poultry Tonic with the feed occasionally; it contains worm destroyer. 


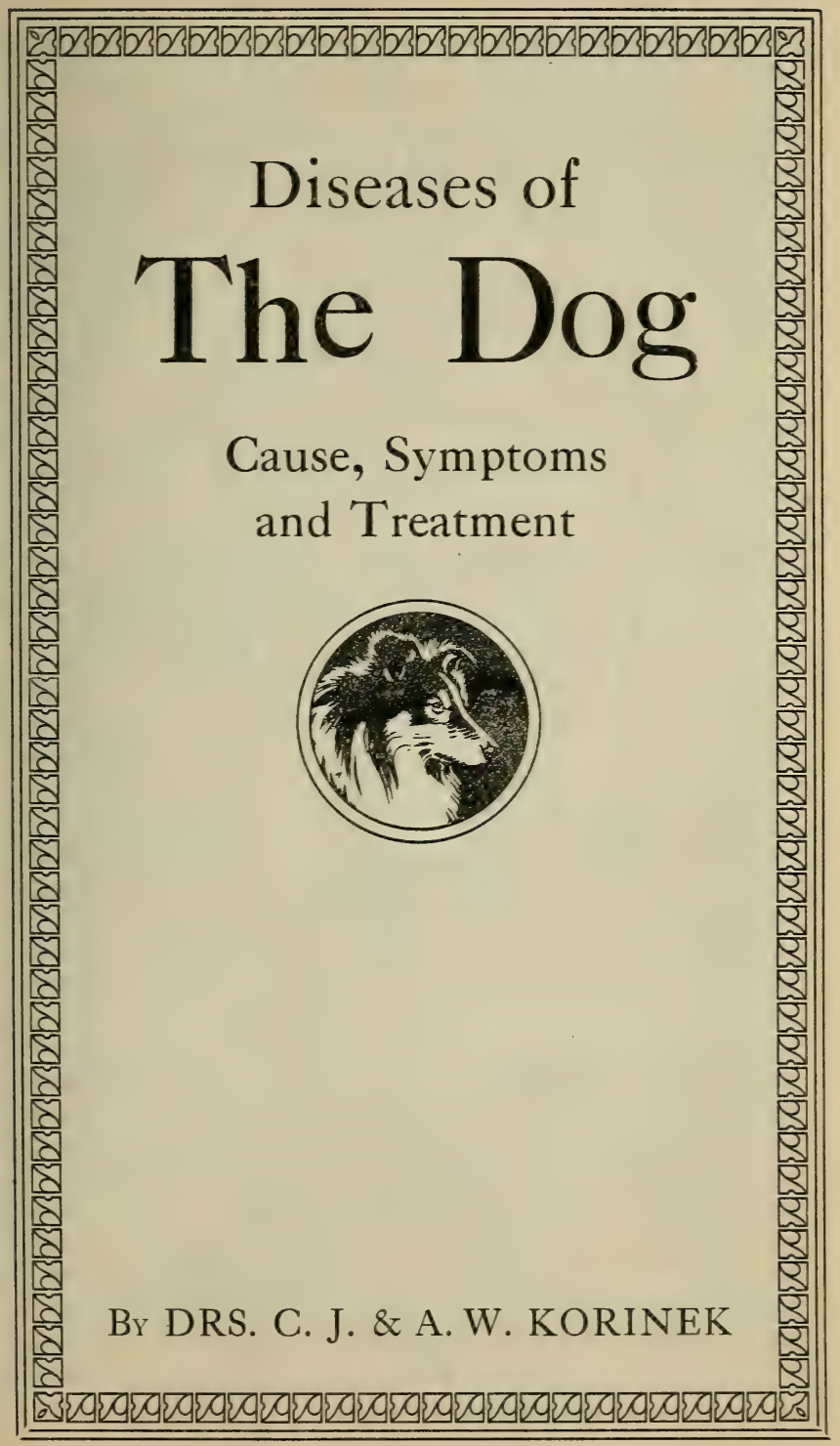




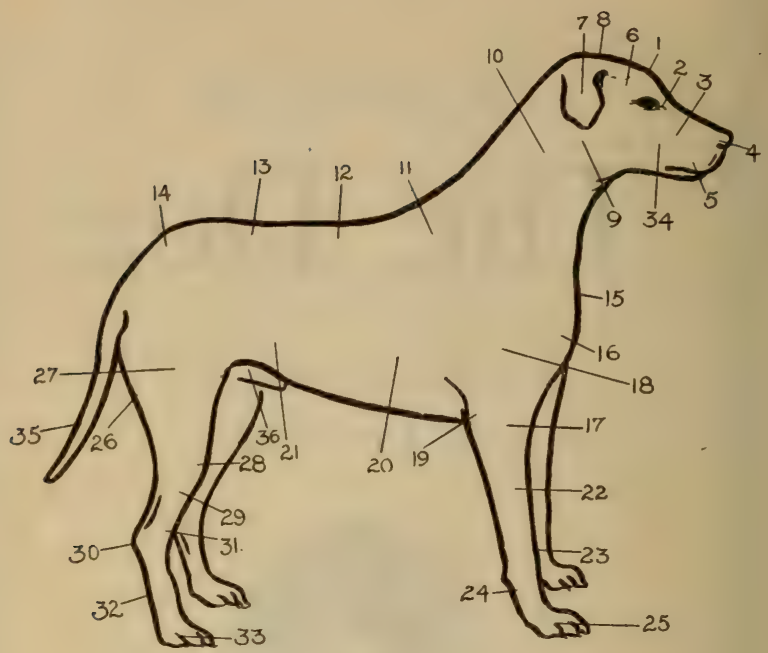

POINTS OF THE DOG

1 Forehead

2 Junction of Nose and Forehead

3 Nose

4 Nostril

5 Muzzle

6 Temple

7 Ears

8 Occiput

9 Posterior Angle of Jaw

10 Neck

11 Withers

12 Saddle

13 Loins

14 Croup

15 Dewlap

16 Brisket

17 Arm

18 Shoulder
19 Point of Elbow

20 Ribs

21 Flank

22 Forearm

23 Knee or Wrist

24 Pastern

25 Toes

26 Buttock

27 First Thigh

$28^{\prime}$ Stifle

29 Second Thigh

30 Point of Hock

31 Front of Hock

32 Pastern

33 Toes

34 Cheeks

35 Tail or Stern

36 Sheath 


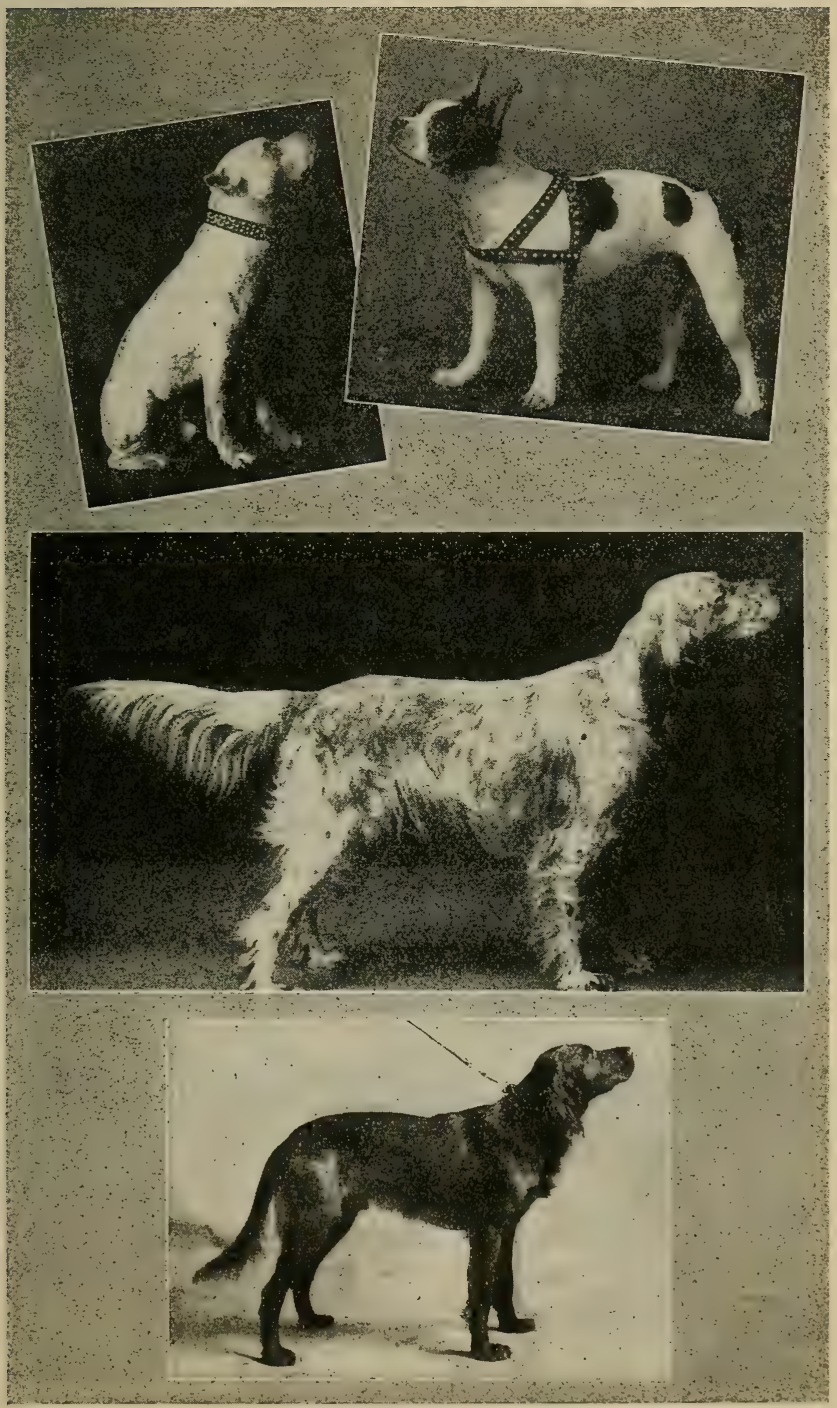

MAN'S FAITHFUL FRIEND 


\section{Abscess of the Ear (Haematoma)}

A common disorder among certain breeds, especially so in dogs with long ears.

CAUSE. Any minor injury, or an itchy sensation that causes the dog to shake his head and flop his ears.

SYMPTOMS. Swelling, heat, and pain; shaking the head violently. On the inner side of the ear a puffy enlargement appears which contains a serous fluid, and which causes much pain on pressure.

TREATMENT. Open the abscess and allow the bloody water to escape. Dress the wound daily with a solution of one part Iodine to five parts water. Fasten the ears to the head by means of a bandage or hood to prevent the animal flopping his ear or scratching the ears with his feet. Give Dr. Korinek's Dog Conditioner which will purify the blood and keep the bowels in good condition.

\section{Accumulation of Tartar on the Teeth}

A common condition among pet dogs and a cause of much annoyance to the owner. ing.

CAUSE. Too much rich food or food that requires no chew-

SYMPTOMS. Teeth are partly covered with a black deposit, and the animal's breath is very offensive. Indigestion is also a symptom.

TREATMENT. Scrape off as much of the tartar as possible, and swab out the mouth with a solution of boracic acid. Allow the dog to chew bones, hard biscuits, etc., and avoid giving food that requires no chewing.

\section{Asthma (Chronic Bronchitis)}

Dilatation of the air spaces in the lungs.

CAUSE. Result of a bad cold or lung fever. In house dogs it may be due to overfeeding, fatness, or old age.

SYMPTOMS. Wheezing or difficult breathing and a spasmodic cough which is aggravated by exercise; offensive breath; constipation.

TREATMENT. A cure is hopeless, but Fowler's Solution of Arsenic (about four drops daily) is beneficial. Feed sparingly and avoid dust and violent exercise. 


\section{Bronchitis}

Inflammation of the membranes lining the Bronchial Tubes.

CAUSE. Exposure to cold; in hunting-dogs it is usually due to hunting in wet fields and swimming cold streams.

SYMPTOMS. Coughing, difficult breathing, usually accompanied by fever and a discharge from the eyes; pressure over the lungs causes pain. The dog sits upon his haunches with drooping head and look of depression.

TREATMENT. Place the dog in warm, comfortable, well-ventilated quarters. Give Dr. Korinek's Dog Distemper Remedy, as directed. Apply mustard plaster over the lungs and feed easily digested and laxative food.

\section{Barrenness}

CAUSE. Failure to breed may be due to physical conditions, such as overfeeding and insufficient exercise, or on the other hand, it may be due to overwork and insufficient supply of food. Forcing to breed will cause the same condition and should never be practiced.

SYMPTOMS. The only symptom present is that the animal is in heat but fails to conceive.

TREATMENT. If due to overwork, rest and good food will usually bring desired results, while if caused by overfeeding and lack of exercise, the dog should be judiciously exercised and fed sparingly. Dr. Korinek's Dog Conditioner acts directly on the generative organs and is a great aid in such cases.

\section{Care of Bitch After Whelping (Bringing Forth Young)}

The mother is in a weakened condition; nourishing her puppies is also a heavy drain on the system and she requires good care. The fetal membrane should be burned or buried at once, for if she eats them she is liable to eat her puppies. Supply her with good rich food, nice, clean, quiet surroundings, plenty of fresh air. Do not allow the animal to be disturbed by the presence of strangers and see that she has a moderate amount of exercise.

\section{Care of New-Born Puppies}

They should have dry, warm, hygienic surroundings, and plenty of soft bedding, which should be changed daily the first week; avoid unnecessary noise and see that they are not picked up or disturbed. 


\section{Care of the Pregnant Mother}

She should have a moderate amount of exercise daily, and a clean, warm place to sleep where she will be quiet and undisturbed. Avoid speaking harshly or kicking or abusing her in any way. Feed on easily digested food, containing plenty of bone, tendon, etc., or the puppies are apt to be undersized and weak; this should be avoided by all means, as undersized dogs are readily classed with curs.

\section{Canker}

There are two different forms, one affecting the external ear or flap, and the other affecting the internal structure of the ear.

CAUSE. Filth and dirt; hunting dogs with big ears are subject to it; in other dogs it is the result of injury to the head. The condition is not serious at first, but as the animal flops his ears or scratches them with his feet it becumes serious and causes much pain.

SYMPTOMS. The dog shakes his head violently, holding it to one side and attempting to scratch the ear with his feet. The ear becomes tender, red and moist and has a disagreeable odor; if the ear be lightly touched it causes severe pain.

TREATMENT. Wash the parts thoroughly with warm water and apply Dr. Korinek's' Mange Remedy. To prevent the dog flopping his ears and scratching them with his feet, a hood of cloth should be made to fit the head and tied on. The ear should be dressed daily. Feed laxative food and give Dr. Korinek's Dog Conditioner as directed upon package.

\section{Care of the Kennel}

Too much stress cannot be laid on the importance of cleanliness and the use of disinfectants, especially where new dogs are constantly being brought in and others sent out; this will prevent the spread of diseases, fleas, mange, etc. For disinfecting kennels use Dr. Korinek's Dip, one part dip to 70 parts water. Dr. Korinek's Lice Powder may be used in the bedding to prevent fleas and lice.

The beds should be kept dry at all times, the sleeping places well-ventilated, and, in cold weather, warm and free from drafts. Bedding should be changed often and the kennel disinfected several times a year.

\section{Dog Distemper}

An infectious disease peculiar to young dogs.

CAUSE. Due to a germ which has not been isolated. Unsanitary surroundings and exposure to extreme cold and wet, are conditions that favor the appearance of this disease. 
SYMPTOMS. Stupidity, lack of spirit, fever, cough, a catarrhal discharge from the nose and eyes, which becomes purulent, pasting the eyelids together and almost closing the nasal passages. There is every sign of unthriftiness; the hair stands and appears dusty, the breath is offensive, and the dog loses flesh rapidly. When given food he will usually vomit. There are three forms of Distemper: the first and most severe affecting the bowels; the second, the respiratory organs; the third, a superficial form affecting the skin. The treatment is the same in all cases.

TREATMENT. Secure warm and sanitary quarters, making the patient as comfortable as possible; if he prefers darkness, humor his choice. Give Dr. Korinek's Dog Distemper Remedy as directed. Coax the animal to eat occasionally, but do not attempt to force food down his throat unless there is danger of starvation. Boiled rice with beef broth or gravy will be relished if the dog is inclined to eat. Allow cold water as often as the animal desires to drink but not too much at one time. Do not lose sight of the fact that nursing plays a very important part in the treatment of this disease.

\section{Fleas-Pulex Serraticeps}

Fleas thrive remarkably in dirty or filthy places. The laying and hatching of eggs is also greatly aided by filth and dirt. The flea infests the dog, cat and sometimes man.

SYMPTOMS. Itching, scratching, redness of the skin, etc.

TREATMENT. Use the same treatment as described under lousiness.

\section{Fits (Epilepsy)}

A nervous disease quite common in the bitch after whelping, and in puppies when teething, or affected with worms.

CAUSE. In the mother it is usually due to constipation, insufficient exercise and nursing too many young; in puppies it is caused by worms or teething.

SंYMPTOMS. Intervals of excitement alternating with periods of stupidity, followed by restlessness and convulsions. The animal's legs become rigid; he foams at the mouth, champs the jaws and may bite anyone who attempts to pick him up or soothe him; in many instances the dog bites his own tongue. After the spasms or convulsions are past the animal seems dazed and seeks a dark place to lie down. 
TREATMENT. Give Dr. Korinek's Dog Physic, according to size and age, as directed. Keep the dog quiet and allow him to fast for two days. Give the following when the condition first appears.

Rx.

Potassii Bromidum, ozs., 1.

Mix and make $5 \mathrm{gr}$. capsules.

Sig. to old dogs, give two capsules and to puppies one capsule, three times daily.

After treatment give Dr. Korinek's Dog Conditioner.

\section{Goiter (Bronchocele)}

CALSE. An enlargement of a ductless gland, very common to dogs, especially puppies, and usually indicates lack of food and proper care, although sometimes well-kept puppies suffer from Goiter. It is often associated with rickets; if this is the case, it is probable that the mother was not properly cared for during pregnancy.

SYMPTOMS. Enlargement in the region of the throat, signs of unthriftiness, stupidity, sleepiness; puppies sometimes die without warning, from suffocation caused by pressure of the growth upon the windpipe. lowing.

TREATMENT. Clip the hair from the part and apply the fol-

Tincture of Iodine....................... 2

Potassium Iodine .......................... 2

Lanolin Ad qs............................ 1

Mix and apply twice a week.

In addition to the above, feed the mother rich food; also lime water, one teaspoonful to a pint of milk: this may also be given to the puppies if they are weaned. Allow plenty of bones to knaw on and see that they have sufficient exercise and are kept clean.
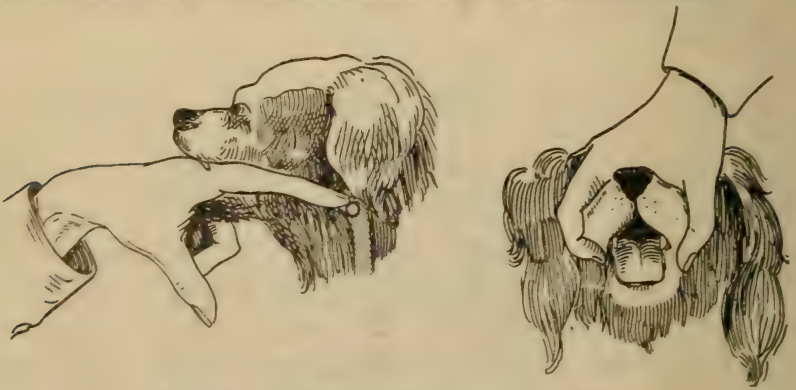

\section{How to Give the Dog a Pill}

With the left hand grasp the nose, push the cheeks between the molar teeth until the mouth is well opened; then with the right hand forefinger push the pill down the throat as far as possible and hold the mouth closed for a few seconds. Sometimes it is advisable to rub the throat downward to cause swallowing. 


\section{Inflammation of the Bowels (Enteritis)}

Very common in dogs but often incorrectly diagnosed.

CAUSE. Impaction; or strangulation of the bowels due to playing, irritating medicine, or indigestible food. Sometimes due to vermifuge medicines given for worms. In hunting dogs it is often caused by severe cold and exertion.

SYMPTOMS. Chills followed by high fever; nose is hot and dry. In the first stages there are signs of colic; the animal lies flat on his side and cries; appears stupid and uninterested in things about him. The bowels fail to move, the urine is scanty, and the tail is drawn close to the body.

TREATMENT. Except in the first stages of the disease, treatment is unsuccessful; gangrene usually sets in and death results.

Injections of warm soapy water several times daily are beneficial. Also give the following:

$\mathrm{Rx}$.

Tincture Opii ........................ 2

Fluid Extracts of Belladonna.............. dr. 4

Aqua Chloroformi ad qs...................... 2

Mix. Sig: One teaspoonful every four hours.

\section{Inflammation of the Bag (Mammitis)}

CAUSE. Common in the bitch and due to injury and exposure when nursing her young.

SYMPTOMS. High fever; the bag becomes hot, swollen, and red, and is very painful to the touch; the milk may be bloody and sometimes contains pus.

TREATMENT. Remove the cause. The puppies should be weaned at once as the milk is unwholesome and nursing is injurious to the mother. Apply Belladonna Liniment two or three times daily; feed laxative food and give Dr. Korinek's Dog Conditioner.

\section{Indigestion}

CAUSE. A common disorder and due to overfeeding or insufficient exercise. May also be due to worms or any food that affects the digestion, as candies, sweets, etc.

SYMPTOMS. Fetid breath, bloating, loss of appetite, dullness. The dog vomits frequently and often eats grass to assist in emptying the stomach. In some cases there are signs of colic.

TREATMENT. Give Dr. Korinek's Dog Physic as directed and rectal injections of warm water, and after the bowels have become normal, give Dr. Korinek's Dog Conditioner. Do not attempt to force the animal to eat; as a rule it does no good and may do harm.

\section{Lousiness}

(Phthiriasis)

CAUSE. Dogs eoming in contact with infected dogs or animals.

SYMPTOMS. The dog invariably scratches and bites himself. The skin becomes very red and irritated and in long haired or shaggy dogs the hair becomes matted. 
TREATMENT. Very beneficial results are derived from the use of Dr. Korinek's Lice Powder, although in some cases it is necessary to use Dr. Korinek's Dip or some other antiseptic, but a 5 per cent. solution of Dr. Korinek's Dip applied with a sponge will usually get the desired results, although some cases have been treated by immersing the dog into a 5 per cent. solution of Dr. Korinek's Dip. It is very necessary to disinfect the sleeping place of the dog as well.

\section{Lung Fever}

Inflammation of the lungs.

CAUSE. Exposure to cold and wet; swimming in cold water or hunting in wet fields.

SYMPTOMS. Coughing, high fever, loss of appetite, peculiar manner of breathing, the cheeks are alternately drawn in and puffed out at every breath. The animal's ears droop; he sits upon his haunches, seldom if ever lying on his side.

TREATMENT. Place in a comfortable place free from cold or draft; give Dr. Korinek's Dog Distemper Remedy, as directed; apply a mustard plaster over the lungs. Allow plenty of fresh water and rich food of a laxative nature.

\section{Piles}

(Hemorrhoids)

CAUSE. Old age, habitual constipation and weakness.

SYMPTOMS. Small elevations or boils on the anus, which become hard and inflamed. The dog usually slides about on his hind quarters.

TREATMENT. Flush the rectum with warm, soapy water; feed laxative food and give Dr. Korinek's Dog Physic, as directed. After the bowels become normal, give Dr. Korinek's Dog Conditioner, which will keep them in good condition and prevent further trouble, if the animal is not too old.

\section{Retention of Milk}

CAUSE. May be due to the bitch becoming pregnant and if this is the case the puppies should be weaned at once and the bag given careful attention, as hard lumps or concretions are apt to form in the teats which will obstruct the flow of milk and perhaps result in abscess, which will necessitate an operation.

TREATMENT. Milk out the bag thoroughly and massage well twice daily. Beneficial results will be obtained from the use of Dr. Korinek's Liniment. 


\section{Rickets}

Malformation of the bone which becomes soft and flexible, causing the affected part to appear out of proportion to the body.

CAUSE. Due to the absence of lime salts in the bone which, instead of being hard and firm, is porous and pliant. Feeding an undue amount of fatty food to the mother will invariably cause Rickets in the puppies. Breeding related parents also has a tendency to produce Rickets in the offspring.

SYMPTOMS. Joints are extremely enlarged and seem to bend under the animal's weight. The bones of the head bulge and appear out of proportion to the rest of the body. In some cases the hocks of the legs come in contact with the ground when the animal walks.

TREATMENT. In puppies: plenty of good milk to which has been added lime water, one teaspoonful of lime water to a pint of milk. Cod liver oil is very beneficial, strengthening the animal and regulating the bowels. Sanitary surroundings and plenty of exercise are very necessary. If the puppies are nursing, give the mother cod liver oil and lime water also, and rich food.

\section{Sore Throat}

\section{(Laryngitis)}

Inflammation of the membranes lining the throat or larynx.

CAUSE. Irritating medicines, exposure to cold, injury from kicks, blows, etc.

SYMPTOMS. Coughing, hacking and vomiting and every appearance of unthriftiness.

TREATMENT. Keep in a clean, comfortable place, free from dust and cold. Give Dr. Korinek's Dog Distemper Remedy as directed, and feed easily-digested food.

\section{Strychnine Poisoning}

CAUSE. Usually given intentionally by some person wishing to kill the animal; often given in meat.

SYMPTOMS. The first sign of strychnine poisoning is a sudden attack of spasms or convulsions. The muscles of the body become stiff, the animal is very excitable; in some cases the lips are drawn back and the dog shows his teeth. The spasms return at intervals, until the animal dies.

TREATMENT. Remove to quiet surroundings, preferably some dark corner where there is absolutely no noise; the stomach should be emptied at once, before too much of the poison has been absorbed by the system. Apomorphine 1/10 gr. injected under the skin will give desired results quickly, but if this is not at hand, give mustard and warm water. After the stomach has been emptied, try to induce sleep by chloroform, ether, or other sleep-producing agents until the spasms are over. 


\section{Sore Mouth}

CAUSE. Eating sharp bones or playing with hard objects or biting the tongue during an attack of convulsions. Teething causes sore mouth in puppies.

SYMPTOMS. Saliva flows freely from the mouth; the animal is usually unable to eat and when the mouth is examined, the membrane is found to be bruised or lacerated.

TREATMENT. Wash the mouth well with a solution of boracic acid, several times daily; give Dr. Korinek's Dog Conditioner, as directed and feed laxative food.

\section{Salmon Poisoning in Dogs}

A common disease and one that usually proves fatal if not treated properly.

CAUSE. The fresh water salmon carries the parasite which produces the disease and when the fish is eaten uncooked by the dog, it results in Salmon Poisoning.

SYMPTOMS. The first signs of disease appear about the ninth or tenth day after the fish has been eaten. The dog lies down most of the time, refuses food but drinks an abundance of water, which is usually followed by excessive vomiting. He lies curled up and shows signs of colic; the movements of the bowels are accompanied by pain and straining and the passages are very offensive in odor. The bowels are loose at first but usually become constipated as the disease progresses, although in some cases bloody diarrhoea continues from the onset until recovery or death. The dog becomes very poor and the membranes of the eyes and mouth are pale and bloodless. The fever is high at the beginning of the disease but the temperature falls below normal later and continues so until the finish.

TREATMENT. The sooner the dog receives treatment, the better are his chances for recovery. Give Dr. Korinek's Salmon Poison Remedy as directed on package and according to size or age and repeat every six hours. Avoid giving water immediately after the dose, as this occasions vomiting and the medicine is lost. Allow plenty of cold water at all other times, however, and when the appetite returns, give soft and palatable food such as boiled rice seasoned with beef broth or gravy, which is very nourishing.

\section{Vomiting}

Expulsion of the contents of the stomach.

CAUSE. May be caused by indigestion, in which case the dog usually eats grass, this being nature's way of emptying the stomach. Worms are a common cause in puppies. 
TREATMENT. If due to indigestion the dog should be starved for a day or two, as it is almost impossible for the animal to retain medicine until the stomach is relieved of the irritant, whatever it may be. Give Dr. Korinek's Conditioner, which will correct indigestion and feed easily digested food, such as boiled rice and beef broth, but avoid cooked meats, bones, etc. If the trouble is due to worms, give Dr. Korinek's Dog Worm Remedy as directed.

\section{Worms}

Dogs are more subject to worms than any other animal and the symptoms are very well-marked in most cases. These symptoms in general are: Irregularity of the bowels, colic and large belly, nervousness, appetite is irregular, hair stands and has a very unthrifty appearance. The dog becomes very poor at the expense of feeding so many. worms. In some cases the dog may whine and even have convulsions or fits. Sometimes the worms work their way into the stomach and are expelled while vomiting. Sometimes worms are passed with the feces and in this case dogs usually slide around on their hind parts.

TREATMENT. In the treatment of worms in dogs, no matter what species they may be, the dog should be isolated or at least tied up during treatment. Withhold all food for twenty-four hours and give Dr. Korinek's Dog Worm Remedy. To puppies give one capsule, to dogs the size of Fox Terriers, two capsules, to dogs the size of pointers and setters three capsules, to dogs the size of a St. Bernard four capsules. This treatment should be repeated in ten days. All feces or manure that is expelled during the treatment should be burned or buried deeply to prevent further spread of the worm.

\section{Tape Worm}

There are several different varieties of the Tapeworm. Each will be described separately.

\section{Tape Worms of the Dog}

(Taenia Marginata)

This is one of the most common tapeworms of the dog; sheep dogs being usually affected.

The head of the worm is wider than the neck. The worm attains the length of about five or six feet and is composed of small segments.

LIFE HISTORY. The ripe segments become detached and pass out with the feces or droppings of the dog. These segments soon become decomposed and myriads of ova are liberated and are spread by the rain, depositing themselves upon the blades of grass or in pools of water and are consumed by sheep. Upon reaching 
the stomach the ovum hatches into a real embyro and penetrates the walls of the stomach and invades the tissues of the liver where it passes a portion of its life cycle, where it finally terminates into a larva. Later it passes from the liver and becomes lodged in the peritonal cavity, where it forms a cyst the size of a hen's egg, taking about two hundred and fifty days to accomplish building a complete cyst and is called a cysticercus tenuicollis. When this cyst is devoured by the dog, the larva is liberated in the digestive organs and upon passing out of the intestines, becomes attached therein and develops into an adult tapeworm in about four or five months.

SYMPTOMS. When the dog is infested by a great number of these worms, digestion is materially affected, irregularity of the bowels, as well as indigestion, nervousness and unthriftiness.

TREATMENT. In case the $d o g$ be a valuable one, he should have special care and good treatment. Give Dr. Korinek's Dog Worm Remedy as directed, according to size and age. This treatment, in most cases, should be repeated in ten days. If the dog is not considered valuable, he should at once be put out of his misery and prevent further spread of the dreaded condition.

\section{Tenia Echinococcus}

One of the worms that ordinarily infest the dog and is found in forty-five to fifty per cent. of post-mortem examinations of dogs. About one per cent. of the hogs slaughtered are found to be affected with a cystic form.

DESCRIPTION OF THE WORM : One-fifth to one-fourth inch in length and very slender, consists of four segments, the worm being about four or five times as long as it is wide. Dogs affected with this worm scatter the segments about the pastures and are spread by the rain, depositing themselves upon the blades of grass or in pools of water where they infest the intermediate host, which is usually hogs, sheep, oxen, or man. The segments of this worm when scattered upon the ground in the droppings of the dog, decay and liberate myriads of ova and when consumed by eating grass or drinking contaminated water, they reach the stomach and a small embyro is liberated which passes through the tissues of the body and becomes lodged in some of the organs, usually the liver or lungs, where it undergoes various changes and finally reaches the larva form. In about five months it becomes a cyst and attains the size of about one inch in diameter, being filled with a colorless, poisonous fluid. Development of this cyst goes on till they become so numerous as to resemble a cluster of grapes. These cysts are called "Echinococcus Polymorphus and are capable of maintaining their vitality for many years and dogs consuming these cysts become carriers of mature worms.

SYMPTOMS. When the cysts affect hogs, sheep, oxen or man the organs affected are naturally interfered with, which causes a 
great loss of flesh, loss of appetite, jaundice, swellings of the head and neck and if cysts are formed in the brain it causes mental disturbance.

SYMPTOMS IN A DOG. Indigestion, nervousness, stupor and sleep, gradual loss of flesh and every appearance of being unthrifty.

TREATMENT OF THE DOG. If the animal is considered valuable he should be treated so. Give Dr. Korinek's Worm Remedy as recommended. Treatment should be repeated within ten days. Through the whole course of treatment the dog should be isolated so as to avoid scattering the segments of the worm. The feces or droppings must be burned or buried deeply to prevent further spreading of the malady. If the dog is worthless, he should be put out of his misery and buried deeply to discontinue scattering the malady.

\section{Tenia Serialis}

Quite uncommon in the United States, but reported prevalent in European countries. This worm infests the dog and the larva, or the cystic form, infests rabbits and rodents.

Symptoms and treatment are the same as other species of the tapeworm.

\section{Tenia Canina}

(Tenia Cucunierina)

This worm is very common to the dogs in the United States and especially so in the Middle West. It is composed of segments and the worm attains the length of about six to twelve inches. The adult worm infests the dog and the larva or cystic form infests the flea or louse of the dog. The dog becomes infested by biting himself when being annoyed by fleas or lice. The puppies usually become infested by nursing the mother. The eggs are taken into the mouth and are hatched in the intestines.

The treatment of this species of tapeworm is the same as the ordinary tapeworm, but great care should be taken to burn or disinfect the quarters where the dog has been treated to prevent reinfestation.

\section{Tenia Corenurus}

This worm is quite uncommon in the dog in the United States, but said to be common in Europe. The adult infests the dog and the larva form infests sheep and rarely cattle, causing "Gid" or "Turn Sickness."

Symptoms and treatment of the dog infested with this form of tapeworm are the same as preceding species. 


\section{Tenia Serreta}

This species of worm infecting dogs, is indeed, very common in the United States. The surface of the worm, as the name indicates, is serrated, or saw-like in form. It is composed of segments about one-half inch long. The length varies greatly. The adult worm infests the dog and the larva or cystic form infests rabbits, causing cysts in the peritoneal cavities or membranes that line the abdominal cavity. The cysts are called Cystercurcus Pisiformis. The dog becomes infested by cating infested rabbits; the rabbits become infested by picking up tice ova on green vegetation, etc.

Symptoms and treatment are the same as Tenia Eschinococcus.

\section{Round Worms}

There are several species of round worm in the dog and each will be described separately.

\section{Ascaris Marginata}

(Maw Worm)

This is one of the most familiar worms that infesis dogs. The worms are about two inches long and are found in the small intestines. About forty-five per cent of dogs have these worms, although they may not show symptoms; nevertheless, when the postmortem examination is held we find these worms present, even in old dogs.

SYMPTOMS. The writer has seen worms so numerous as to cause a complete closure of the bowels. The worms are occasionally passed out with feces and sometimes they work their way into the stomach and are forced out when vomiting. Frequent colicky pains; the belly becomes abnormally enlarged and the hair is very unthrifty. The animal is usually very poor and has a ravenous appetite.

TREATMENT. After fasting for twenty-four hours give Dr. Korinek's Worm Remedy: To puppies one capsule, dogs the size of a Fox Terrier two capsules, dogs the size of Pointers and Setters three capsules, and dogs the size of a St. Bernard, four capsules. Keep the animal isolated while being treated and repeat the treatment in ten days. All fecal matter and manure where dogs have been treated should be disinfected and the bedding, etc., burned.

\section{Uncinaria Canina}

This is a very common species infesting dogs, and is quite fatal to puppies. It is threadlike in form, white in color and from onehalf to three-quarter inch long. Life history of this worm is similar to preceding species. The adult worm infests the dog, cat and fox. 
SYMPTOMS. Causes more pain, perhaps than any other worm infesting puppies. There will be much whining, bloating and derangement of the digestive organs, diarrhoea, loss of appetite and nervousness. The animal becomes poor and may succumb to the disease if relief is not afforded.

TREATMENT. Give Dr. Korinek's Dog Worm Remedy capsules as directed upon package and following with a physic. Repeat this treatment again in ten days. All the necessary precautions in the treatment of dogs infested with worms should be practiced.

\section{Trichocephalus Depressiuisculus}

(Sometimes called the Whip Worm.)

Fortunately this worm does not affect the dogs of this country to any great extent. The worm, as the name indicates, is "whip" shaped, the tail being coiled up like a watch spring. It measures from one and one-quarter inch to one and one-half inch in length and infests the large intestines. No doubt the dog picks up the ova as he hunts or smells about upon the ground, getting the eggs into his mouth and later they are hatched in the intestinal tract.

Under favorable conditions of warmth the ova of this worm will hatch in from five to six months.

SYMPTOMS. The dog becomes anemic, or the membranes lining the mouth and eyes become very pale. The animal is very thirsty, there is always derangement of the digestive organs, constipation and sometimes diarrhoea, but most likely the latter. Canina.

TREATMENT. Same treatment is used as for the Uncinaria

\section{Whelping}

Keep the mother in a quiet room, free from noise or excitement of any kind. When the puppies are born, they should be kept warm, dried if necessary and encouraged to nurse as soon as possible. 



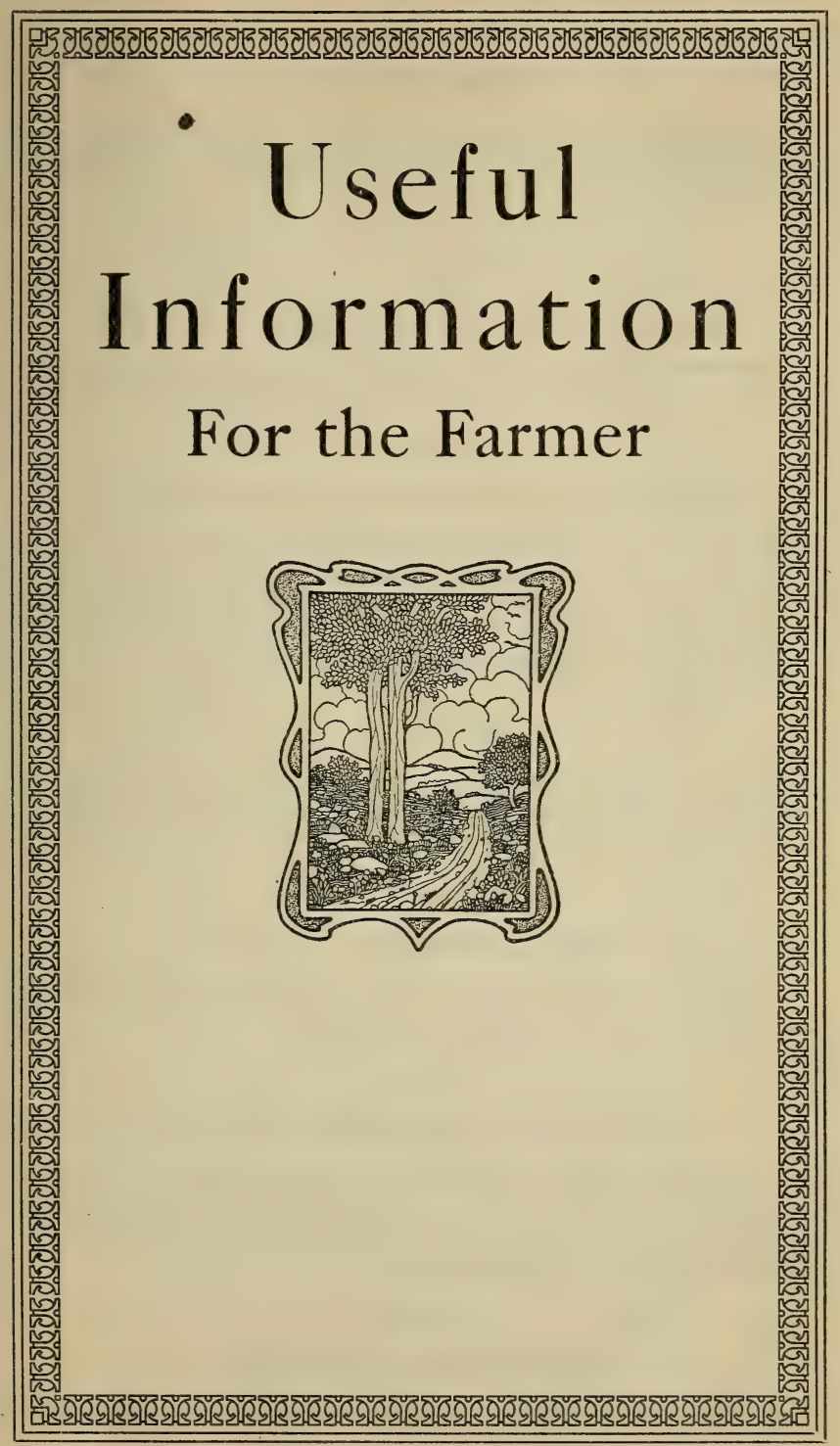




\section{RESPIRATION.}

The number of respirations per minute varies with the different classes of animals; as a rule, the larger the animal, the slower the respiration.

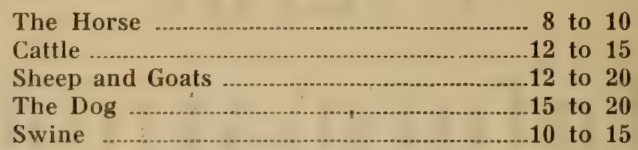

The rate of breathing is increased from the processes of digestion immediately after eating, or may increase from exercise.

\section{NORMAL TEMPERATURE OF THE HORSE.}

From 2 to 5 years old the temperature is $100-6^{\circ}$ Fahr.

From 5 to 10 years old the temperature is $100-4 \circ \mathrm{Fahr}$.

From 10 to 15 years old the temperature is $100-\circ$ Fahr.

From 15 to 20 years old the temperature is 98.4 to $100-2 \circ \mathrm{Fahr}$.

Sex appears to slightly influence temperature:

Stallion $100^{\circ}$ Fahr.

Mare $100.8^{\circ}$ Fahr.

Gelding 100.4० Fahr.

The time of day when temperature is taken is important, the lowest body temperature being at $4 \mathrm{a} . \mathrm{m}$., and the highest at $6 \mathrm{p} . \mathrm{m}$. New born foals' temperature will run from $102 \circ$ to $104 \circ \mathrm{Fahr}$.

\section{TEMPERATURE OF CATTLE.}

Normal temperature is from $101.8^{\circ}$ to $102^{\circ}$ Fahr. Compared with the horse the daily variations are small.

\section{TEMPERATURE OF SHEEP AND GOATS.}

In these animals the greatest variation in temperature occurs, viz.: $100.9^{\circ}$ to $102.8^{\circ} \mathrm{Fahr}$. In the majority of cases the temperature probably will be between $103.6^{\circ}$ to $104.4^{\circ} \mathrm{Fahr}$. The cause of this variation is unknown.

\section{TEMPERATURE OF SWINE.}

The average temperature is $103.3^{\circ}$ Fahr., varying from $100.9^{\circ}$, to $105.4^{\circ} \mathrm{Fahr}$. 


\section{TEMPERATURE OF THE DOG.}

The dog is subject to important variations depending on the external temperature; it varies from $99.5^{\circ}$ to $103^{\circ} \mathrm{Fahr}$, Feeding will increase the temperature, and it is also higher toward evening.

\section{PULSE THROBS PER MINUTE.}

The Horse
Cattle _...
Sheep and Goats
The Dog
Swine

The pulse in the young is much more rapid than in the adult animal; that of a foal at birth beats 100 to 120 per minute, while that of a calf will go to 130 per minute. In old age the pulsation becomes reduced and the arteries much weaker. The pulse rate in large animals is less than in smaller ones, as for instance, an elephant's pulse rate is from 25 to 28 beats per minute. The more rapid the pulse, the greater the quantity of blood in circulation.

\section{THE AGE WHEN DIFFERENT ANIMALS COME INTO HEAT (PUBERTY).}

Mare
Cow
Sheep and Goats
Sow
Bitch

The time of year the different animals come into heat (Oestrum) Mare, April to July...................................... Duration 1 to 7 days Cow, any time but usually spring........................ Duration 1 to 2 days Sheep and Goats, September (Dorset Horns

twice yearly)

Duration 1 to 2 days Sow, any time and twice a year....................... Duration 2 to 3 weeks Bitch, any time and twice a year....................Duration 2 to 3 weeks Cat, any time and three times a year......................Duration indefinite

The length of time the mothers carry their young (Gestation).

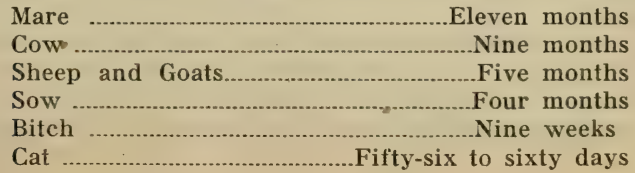




\section{AVERAGE PERIOD OF INCUBATION.}

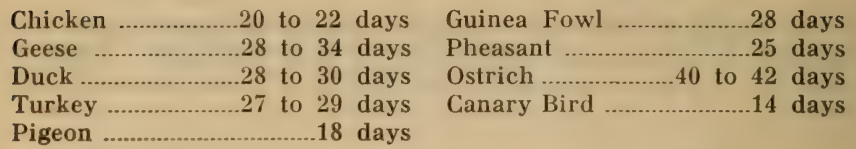

\section{SOME FACTS ABOUT STOCK FOOD.}

Water is contained in all foods and feeding stuffs. The amount varies from 8 to 15 pounds per 100 pounds of such dry materials as hay, straw, or grain, to 80 pounds in silage and 90 pounds in some roots.

Dry matter is the portion remaining after removing or excluding the water.

Ash is what is left when the combustible part of a feeding stuff is burned away. It consists chiefly of lime, magnesia, potash, soda, iron, chlorin, and carbonic, sulphuric, and phosphoric acids, and is used largely in making bones. Part of the ash constituents of the food is stored up in the animal's body; the rest is voided in the urine and manure.

Protein (nitrogenous matter) is the name of a group of substances containing nitrogen. Protein furnishes the materials for the lean flesh, blood, skin, muscles, tendons, nerves, hair, horns, wool, casein of milk, albumen of eggs, etc., and is one of the most important constituents of feeding stuffs.

Gluten is the name given to one of the most important of the nitrogenous substances classed together under the general term "protein." "Wheat gum," obtained by carefully chewing wheat, is a familiar example. It is the gluten of flour that gives consistency to the dough.

Carbohydrates - The nitrogen-free extract and fibre are often classed together under the name of carbohydrates. The carbohydrates form the largest part of all vegetable foods. They are either stored up as fat or burned in the body to produce heat and energy. The most common and important carbohydrates are sugar and starch.

Fibre, sometimes called crude cellulose, is the framework of plants, and is, as a rule, the most indigestible constituent of feeding stuffs. The coarse fodders, such as hay and straw, contain a much larger proportion of fibre than the grain's, oil cakes, etc.

Nitrogen-free extract includes starch, sugar, gums, and the like, and forms an important part of all feeding stuffs, but especially of most grains.

Fat, or the materials dissolved from a feeding stuff by ether, is a substance of mixed character, and may include, besides real fats, wax, the green coloring matter of plants, etc. The fat of food is either stored up in the body as fat or burned to furnish heat and energy. 


\section{FARM BARN HINTS.}

Pull the plug from the pump pipe before heavy freezing comes. It will save thawing out the pump some frosty morning, or perhaps the price of a new pump.

The cattle need not stand at an empty trough on a hot day if you pump with a gasoline engine.

If you give the boy a calf, let him do the selling, and let him use the money. A hint that he buy two or three pigs or another calf might start him right.

Oil the hayfork carrier and pulley blocks before starting to fill the mows.

Your victuals are flat if not seasoned with salt. The cattle have a sense of taste too.

Some horses had rather stand up all night than lie on a hard, bare floor. They'll do better work next day if you bed them well at night.

Good cows are often as particular about their bed.

It costs many bushels of corn to keep poorly sheltered hogs warm with their own body heat. It's cheaper to build a warm hog house.

Pet the colt. It helps to make a gentle horse.

Your stock will look better inside a good barnyard fence.

Feed the calf warm milk direct from the cream separator. Don't forget the handful of oil meal to replace the butter fat.

Keep the cats at the barn. When the cats are away the mice will play havoc in the feed bin.

If the Monday dinner is late, perhaps the wife is doing the work of a gasoline engine in turning the washer and wringer.

You are repaid for keeping a well cultivated garden when meal time comes.

If you are having a "streak of bad luck" improve your farm management.

\section{TO REMOVE DAMPNESS AND MOULD IN CELLARS.}

\section{(Colorado Agricultural College)}

When a cellar or basement becomes objectionably damp the condition may be corrected by placing anhydrous calcium chloride about in old tin cans. A pound of the salt is sufficient to treat a large cellar.

The moisture, being absorbed by the chloride collects in the tins. However the salt is not thus rendered useless, but, after the moisture is driven off over a strong fire, may be used again.

To destroy mould in a cellar, grind unslaked lime to a fine powder and dust it over the infested walls and blow it into cracks and crevices. 
FIRST AID TO THE INJURED.

Burns and Scalds.-Cover with cooking soda and lay wet cloths over the injured part. Household ammonia applied immediately is excellent. Other remedies are: White of egg and olive oil. Olive oil or linseed oil plain or mixed with chalk and whiting. Sweet or olive oil and lime water.

\section{Lightning.-Dash water over the person struck.}

Sunstroke.-Ioosen clothing. Get patient into the shade and apply ice-cold water to the head. Keep the head in elevated position.

Stings of insects.-Apply weak ammonia, oil, salt water, iodine.

Mad Dog or Snake Bite.-Tie a cord tightly above the wound. Suck the wound and cauterize with caustic or white-hot iron immediately, or cut out adjoining parts with sharp knife. Give stimulants -whiskey or brandy being the most effective.

Fainting.-Place flat on back. Allow fresh air to circulate, and sprinkle with water. Place the head lower than the rest of the body.

Cinders in the Eye.-Rub the other eye. Roll soft paper up like a lamp-lighter and wet the tip to remove, or use a medicine dropper to draw it out.

Fire in One's Clothing.-Don't run-especially not downstairs or out of doors. Foll on a carpet, or wrap in a woolen rug or blanket. Keep the head down, so as not to inhale the flame.

Drowning. 1. Loosen the clothing, if any. 2. Empty the lungs of water by laying the patient on his stomach, and lifting him by the middle, so that the head hangs down. Jerk the body a few times. 3. Pull the tongue forward, using handkerchief, or pin with string, if necessary. 4. Imitate respiration by alternately compressing and expanding the lower ribs about twenty times a minute. Alternately raising and lowering the arms from the sides up above the head, gently but persistently, will stimulate the action of the lungs. 5. Apply warmth and friction to extremities. $6 .{ }^{-1}$ By holding tongue forward, closing the nostrils, and pressing the "Adam's apple" back (so as to close entrance to stomach) direct inflation may be tried. Take a deep breath and breathe it forcibly into the mouth of patient, compressing the chest to expel the air. Repeat this operation. 7. Don't give up! People have been saved after hours of patient, active effort. 8 . When breathing begins, get the patient into a warm bed, give warm drinks, or spirits by teaspoonfuls. Let there be plenty of fresh air and quiet.

Suffocation from Inhaling Illuminating Gas.-Get into the fresh air right away and lie down. Keep warm. Take ammonia-20 drops to a tumblerful of water, at frequent intervals. Also 2 to 4 drops of tincture of nux vomica every hour or two for five or six hours. 


\section{WHAT TO DO TILL THE DOCTOR COMES.}

\section{Extracts from instructions "For First Aid to Injured."}

A Shock.-If faint and cold, give stimulant in small doses, once in fifteen or twenty minutes, and secure warmth by external application or rubbing.

Bleeding from Wound.-If from an artery, stop the current of blood to the wound by putting a compress or cloth pad over the artery. Fasten it firmly by a handkerchief or bandage, which may be tightened by twisting in a stick as a binder. The location of the artery can generally be determined by the throbbing sensation. If from a vein, apply pressure directly over the wound or by exposure or application of cold water.

Perchloride of iron solution may be applied with cloth or lint. Keep the part elevated.

Wounds.-The part should be properly cleansed of all foreign matter, the edges brought together and fastened with strips af plaster; apply some anodyne solution; give stimulant, laudanum with brandy, if necessary.

Bruises.-Apply tincture of arnica and wormwood or hamamelis; keep well covered and warm.

Poisoned Wounds, from bite of animals.-Treatment should be prompt. If possible, suck the wound thoroughly two or three minutes; cauterize with either nitric acid, chloride of zinc or nitrate of silver, use whisky freely internally.

Stings of Insects.-Apply spirits of ammonia.

Poisons.-General Directions.-Give an emetic as soon as possible; tablespoonful of powdered mustard in a tumbler of warm water, or twenty grains of ipecac; after vomiting, give freely of warm drinks.

\section{INTEREST.}

To find the interest on any sum, at any rate per cent and for any length of time: First-Multiply the principal by the rate per cent expressed in hundredths: this will give the interest for one year. Second-Find the number of days remaining, by consulting the calendar, and multiply the principal by as many hundredths as there are days, and for 3 per cent, divide the product by 120 ; for 4 per cent, divide by 90 ; for 5 per cent, divide by 72 ; for 6 per cent, divide by 60 ; for 7 per cent, divide by 52 ; for 8 per cent, divide by 45 ; for 9 per cent, divide by 40 ; for 10 per cent, divide by 36 , and for 12 per cent, divide by 30 . This will give the interest for the days. Third-Add the two items of interest, and the sum will be the entire interest. 


\section{HINTS ON RIGHT LIVING.}

Air and Exercise.-Take abundance of fresh air, especially in your sleeping room at night. Avoid drafts; shut them out or move aside.

Exercise daily in the open, preferably in the performance of useful labor of some kind. Walking about just for the sake of walking is not the best of exercise. Walk erect with the chest up and the chin held in, and practice deep, full.breathing through the nostrils, not through the mouth.

Bathing.-Take a full bath in moderately hot water and soap, followed with cold water and a vigorous rub, once" or twice a week for cleanliness. Always finish the bath with cold water to give vigor, close the pores and insure against taking cold.

Take a plunge in cold wated every morning, or a cold sponge or towel bath, wipe dry and then induce a perfect reaction or warm, healthy glow by a friction rub with a coarse Turkish towel, by slapping and by exercise.

Take this morning tonic regularly and you will seldom, if ever, take a cold and you will need but little extra clothing in winter.

Proper Clothing.-Never expose yourself by wearing too little clothing; but do not form the habit of bundling to keep warm. Keep the chest and extremities well protected. Do not wear a chest protector that is impervious to air.

Undergarments should be such as will readily absorb perspiration, thus keeping the body dry and free from chilling.

Always exchange wet or damp clothing for dry before chilling.

When chilled keep exercising or take a hot foot and leg bath immediately, if possible.

Do not retard the circulation of the blood or the natural development of the body by wearing tight-fitting garments. Suspend the clothing from the shoulders, leaving the waist and hips free.

Temperate Habits.-Do not engage in harmful amusements, nor keep late hours, nor take on habits that weaken either mind or body.

Foods.-With proper substitutes, it is better to cut out flesh meats altogether.

Have regular meals of wholesome, nutritious, well prepared and well cooked foods.

Give attention to the right combination of foods.

Eat only when hungry and only enough to satisfy hunger. Masticate thoroughly.

Take good care of your teeth.

Do not use strong condiments, as mustard, pepper-sauce, etc.

Do not discuss the food question in your own mind or with others while eating. 
Good Cheer.-Do right and have á clear conscience and be cheerful and enjoy life.

Never give place for a moment to anger, worry or gloom, or to thinking or speaking ill of others.

Cultivate friendship. Be broad-minded and generous in your estimate of people whom you do not like, if there be any such.

Declare perpetual peace with yourself and all things else, animate and inanimate, including the weather.

He that is of a merry heart hath a continual peace.-Solomon.

Excluding Selfishness.-Think little of self, but much of the welfare of others. Devote your life and means to the good of humanity. Connect this life with the life and world to come.

Life and the Body.-Life is the happiest gift of God, and the human body is the best of Nature's handiwork. It is perfect in design and wonderful in construction. Carelessness, aided by ignorance, is responsible for all its diseases and all its defects. A regulated system of health would astound mankind with results both marvelous and enjoyable.-Hale.

\section{ANTIDOTES FOR POISONS.}

First. Send for a physician.

Second. Induce vomiting by tickling throat with feather or finger, drinking hot water or strong mustard and water. Swallow sweet oil or whites of eggs.

Acids are antidotes for alkalies, and vice versa.

\section{SPECIAL POISONS AND ANTIDOTES.}

Acids-Muriatic, Oxalic, Acetic, Sulphuric (Oil of Vitriol), Nitric (Aqua Fortis).

Antidotes-Soapsuds, magnesia, lime water.

\section{Prussic Acid.}

Antidotes-Ammonia in water. Dash water in face.

Carbolic Acid.

Antidotes-Whisky or dilute grain alcohol if obtainable, flour and water, mucilaginous drinks.

Alkalies-Potash, Lye, Hartshorn, Ammonia.

Antidotes-Vinegar or lemon juice in water.

Arsenic-Rat Poison, Paris Green.

- Antidotes-Milk, raw eggs, sweet oil, lime water, flour and water. 
Bug Poison-Lead, Salt Petre, Corrosive Sublimate, Sugar of Lead, Blue Vitriol.

Antidotes-Whites of eggs, or milk in large doses.

\section{Chloroform-Chloral, Ether.}

Antidotes.-Dash cold water on head and chest, artificial respiration.

Carbonate of Soda-Coperas, Cobalt.

Antidotes-Soapsuds and mucilaginous drinks.

Iodine-Antimony, Tartar Emetic.

Antidotes-Starch and water astringent infusions, strong tea.

Mercury and its Salts.

Antidotes-Whites of eggs, milk, mucilages.

Opium-Morphine, Laudanum, Paregoric, Soothing Powders or Syrups.

Antidotes-Strong coffee, hot bath. Keep awake and moving at any cost.

\section{TABLOID RECIPES.}

Ink stains removed by solution of oxalic acid.

Acid stains, apply pearl ash, boil in soap water.

Iron stains, use oxalic acid or buttermilk.

Fruit stains, wet cloth, hold over burning sulphur.

Grease spots, equal parts ether, ammonia and alcohol.

Rust in iron removed by kerosene oil.

Paint or glass cleaned by using strong hot vinegar.

Smoked mica cleaned by washing with vinegar.

Dry putty removed by applying hot metal.

Steel cleaned by unslaked lime.

Metals cleaned by rottenstone and spirits of turpentine.

Marble cleaned by strong lye and quicklime.

Brass cleaned by half ounce alum boiled in one pint of water.

Paint cleaned by using common whiting.

Silver cleaned by weak solution of ammonia.

Gold cleaned by soap and water, dry in magnesia.

Wells cleaned by throwing in a peck of unslaked lime.

Colors in fabrics set by spoonful oxgall to gallon water.

Lamp chimneys toughened by boiling in weak brine.

Lamp smoking prevented by soaking wick in vinegar.

Caneseat chairs tightened, sponge with hot water.

Mold prevented by small quantity of carbolic acid.

Steel pens non-corroding, bit of iron in ink.

Wood hardened by boiling in olive oil ten minutes.

Ring on finger removed by holding in cold water. 


\section{MISCELLANEOUS WEIGHTS AND MEASURES.}

Troy Weight.

- 24 grains 1 pwt.

20 pwt. 1 ounce

21 ounces 1 pound

Used for weighing gold, silver and jewels.

Apothecaries' Weight.

20 grains

3 scruples

1 scruple

8 drams 1 dram

12 ounces 1 ounce 1 pound

The ounce and pound in this are the same as in Troy Weight.

Avoirdupois Weight.

27 11-32 grains

.1 dram

16 drams

1 ounce

16 ounces

25 pounds 1 pound

4 quarters 2,000 pounds 1 quarter 2,240 pounds $1 \mathrm{cwt}$. 1 short ton 1 long ton

2 pints

8 quarts

4 pecks

36 bushels

Dry Measure.

1 chaldron
-1

Liquid Measure.

4 gills 1 pint

2 pints 1 quart

4 quarts

$31 \frac{1}{2}$ gallons

2 barrels

1 gallon

1 barrel

1 hogshead

Time Measure.

60 seconds 1 minute

60 minutes

1 hour

24 hours

7 days 1 day $28,29,30$ or 31 days 1 week ( 30 days 365 days 366 days 1 calendar month 1 month in computing interest) 1 year 1 leap year

60 seconds

Circular Measure.

60 minutes

1 minute

1 degree

30 degrees 1 sign

90 degrees 1 quadrant

4 quadrants 12 signs or 360 degrees. 1 circle 
1728 Cubic Measure.

27 cubic feet.

128 cubic feet 1 cubic foot 1 cubic yard

40 cubic feet 1 cord (wood). 2150.42 cubic inches 231 cubic inches 268.8 cubic inches

1 cubic foot 1 ton (shipping) 1 standard dry bushel 1 standard liquid gallon 1 standard dry gallon about 4-5 of a bushel

6 feet

Mariners' Measure.

12 faths.

$71 / 2$ cable lengths

5280 feet

1 fathom

6085 feet

1 cable length 1 mile 1 statute mile 1 nautical mile

12 inches

Long Measure.

3 feet 1 foot

$51 / 2$ yards

40 rods 1 yard

8 furlongs 1 rod

3 miles 1 furlong 1 statute mile .1 league

$21 / 4$ inches Cloth Measure.

4 nails 1 nail

4 quarters

1 quarter 1 yard

24 sheets

20 quires

2 reams

5 bundles 1 quire

Paper Measure.

Miscellaneous.

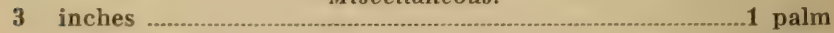

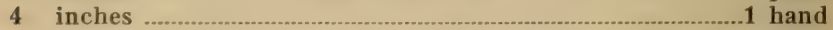

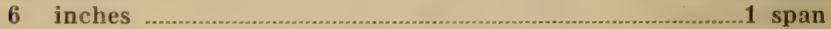

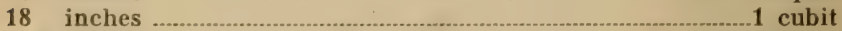

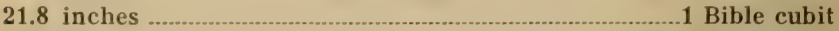

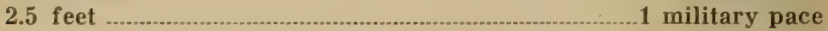

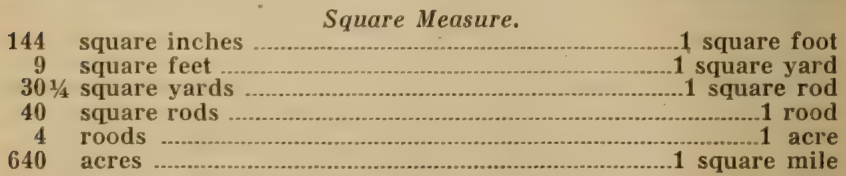

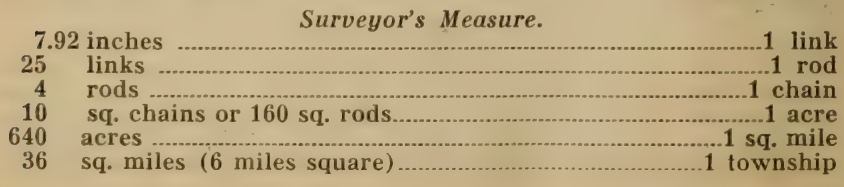




\section{WEIGHTS OF EVERYDAY THINGS.}

A barrel of flour weighs …

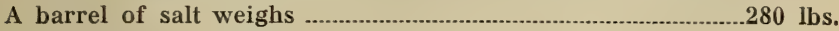

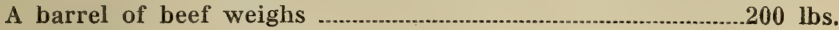

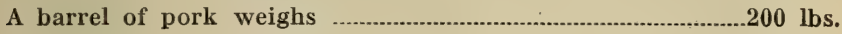

A barrel of fish weighs ................................................

A keg of powder equals........................................................... 25 lbs.

A stone of lead or iron equals ..................................................... 14 lbs.

A pig of lead or iron equals.................................................211/2 stone

Anthracite coal, broken-cubic foot averages........................... 54 lbs.

A ton, loose, occupies..................................................40-43 cubic feet

Bituminous coal, broken-cubic foot averages........................... 49 lbs.

A ton, loose, occupies ......................................... 40-48 cubic feet

Cement (Hydraulic) Rosendale, weight per bushel.................... 70 lbs.

Cement (Hydraulic) Louisville, weight per bushel..................... 62 lbs.

Cement (Hydraulic) Portlana, weight per bushel.................... 96 lbs.

Gypsum, ground, weight per bushel........................................ 70 lbs.

Lime, loose, weight per bushel

Lime, well shaken, weight per bushel...................................... 80 lbs.

Sand at 98 lbs. per cu. foot, ner bushel................................1221/2 lbs.

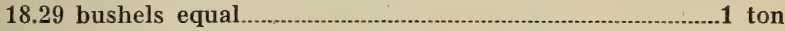

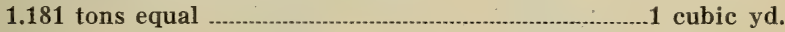

\section{LEGAL WEIGHT OF VARIOUS COMMODITIES.}

(MINIMUM WEIGHT, BY U. S. STATUTE)

\begin{tabular}{|c|c|}
\hline Pounds Per Bushel & 20 - \\
\hline pples, dried .... & Lime, unslaked \\
\hline arley ............................. & Malt ........ \\
\hline eans, castor 46 & Millet seed ........ \\
\hline ans, white ......... & Oats \\
\hline luegrass seed... & Onions \\
\hline 20 & Peac \\
\hline 48 & Pea \\
\hline ....... 60 & Pea \\
\hline ........ 80 & ...... 60 \\
\hline 56 & sweet \\
\hline 70 & Ry \\
\hline ..... 48 & $\mathrm{Sa}$ \\
\hline ........... 56 & Salt, coarse \\
\hline air, plastering ................................ & Timothy seed \\
\hline Hemp Seed ........................ 44 & Turnips ............. \\
\hline ungarian grass seed...... & Wheat \\
\hline
\end{tabular}




\section{WEIGHTS AND HOUSEHOLD MEASURES.}

45 drops of water make a teaspoonful.

1 teaspoonful equals 1 fluid dram.

1 dessertspoonful equals 2 teaspoonfuls, or 2 drams.

1 tablespoonful equals 2 desertspoonfuls, or 4 teaspoonfuls.

2 tablespoonfuls equals 8 teaspoonfuls, or 1 fluid ounce.

1 common size wineglassful equals 2 ounces or $1 / 2$ gill.

1 common size tumbler holds $1 / 2$ pint.

A small teacup is estimated to hold 4 fluid ounces or one gill.

1 pound of wheat is equal to about a pint.

1 pound and 2 ounces of Indian meal is equal to 1 quart.

1 pound of sugar is equal to about 1 pint.

1 pint of pure water is about one pound.

\section{WEIGHT OF FEEDS PER QUART.}

1 Frequently the farmer wishes to feed a given weight of this, that or the other stuff and has no scales at hand to weigh it. If he has a quart measure handy, he can use it to measure out the required weight. The quart weight of various feeds is as follows:

Cottonseed meal, 1.5 pounds; linseed meal, old process, 1.1 pounds; giuten meal, 1.7 pounds; gluten feed, 1.2 pounds; wheat bran, coarse, .j pound; wheat middlings, coarse, .8 pound, and fine, 1.1 pounds; mixed wheat feed, .6 pound; cornmeal, 1.5 pounds; oats, 1.2 pounds; rye bran, .6 pound; H. O. dairy feed, .7 pound, and Victor corn feed, .7 pound per quart.

\section{PRACTICAL MEASUREMENT OF LAND.}

To find the number of acres in any rectangular piece of land, multiply the length and breadth in rods together, and divide by $\mathbf{1 6 0}$ (the number of square rods in an acre) and the result will be the required answer.

When one side and perpendicular to that side from the opposite angle are given, take one-half the product of the side and perpendicular, and divide by 160 .

When three sides are given, from half the sum of the three sides subtract each side separately; muitiply the half sum and the three remainders together; the square root of the product divided by $\mathbf{1 6 0}$ will give the number of acres in the field.

When the piece of land is in the shape of a trapezoid, take onehalf the product of the sum of the parallel sides and the perpendicular between those sides, and divide by 160 .

To find the area of any straight-sided piece of land, divide the latter into convenient parts, find the area of every part, and the sum will be the area of the field.

In general, the parts into which the field can be most conveniently divided will be triangles, but in some cases we may have a rectangle or a trapezoid, whose areas may be found by the preceding rules. The area of a right-angle triangle equals one-half the product of the two short sides. 


\section{WHEN TO PLANT SEEDS.}

The seeds of farm crops may be divided into two classes according to the temperature at which they will germinate or sprout readily and can be safely planted, and the blossoming time of fruit trees can be taken as a more certain guide than any arbitrary calendar date.

Class A.-Those seeds that will germinate or sprout at an average temperature of forty-five degrees in the shade or at about the time the peach and plum trees blossom:

$\begin{array}{lll}\text { Barley } & \text { Beet } & \text { Parsley } \\ \text { Oats } & \text { Carrot } & \text { Parsnip } \\ \text { Rye } & \text { Cabbage } & \text { Onion } \\ \text { Wheat } & \text { Cauliflower } & \text { Pea } \\ \text { Red clover } & \text { Endive } & \text { Radish } \\ \text { Crimson clover } & \text { Kale } & \text { Turnip } \\ \text { Grasses } & \text { Lettuce } & \text { Spinach }\end{array}$

These can be planted with safety in the spring as soon as the ground can be prepared, and some of them, if planted in the fall, live through the winter.

Class B.-Those seeds that will germinate or sprout at an average temperature of sixty degrees in the shade, or when the apple trees blossom:

$\begin{array}{lll}\text { Alfalfa } & \text { Soy bean } & \text { Squash } \\ \text { Cow Pea } & \text { Pole bean } & \text { Cucumber } \\ \text { Corn } & \text { String bean } & \text { Pumpkin } \\ \text { Cotton } & \text { Melon } & \text { Tomato } \\ \text { Eggplant } & \text { Okra } & \text { Pepper }\end{array}$

\section{LENGTH OF GERM LIFE IN SEEDS.}

$\begin{array}{r}\text { Vegetables- } \\ \text { Cucumber }\end{array}$
Melon




\section{GERMINATING TABLE OF GARDEN SEEDS.}

\begin{tabular}{|c|c|c|c|}
\hline & \multicolumn{2}{|l|}{ Days } & Days \\
\hline & & Lettuce & . 6-8 \\
\hline D & $7-10$ & Onion & $7-10$ \\
\hline Cabbage & $5-10$ & Pea .... & $6-10$ \\
\hline Carrot. & $. .12-18$ & Parsnip & $10-20$ \\
\hline Cauliflower & $5-10$ & Pepper & $9-14$ \\
\hline Celery & $10-20$ & Radish & $3-6$ \\
\hline r & $5-8$ & Salsify & $7-12$ \\
\hline ce & $6-10$ & Tomato & $6-12$ \\
\hline dive & $5-10$ & Turnip & $4-8$ \\
\hline
\end{tabular}

QUANTITY OF SEED REQUIRED FOR A GIVEN NUMBER OF HILLS.

Hills

Pole beans................... $1 \mathrm{lb}$ to 100

Corn …..................... llb. to 300

Cucumbers ……......... oz. to 50

Watermelons …............. $1 \mathrm{oz}$. to 30

Hills

Muskmelons 1 oz. to 60

Okra $1 \mathrm{oz}$. to 100

Pumpkins $1 \mathrm{oz}$. to 30

Squash 1 oz. to 30

QUANTITY OF SEED REQUIRED FOR A GIVEN NUMBER OF PLANTS.

\begin{tabular}{|c|c|c|c|}
\hline \multirow[b]{2}{*}{ Asparagus } & \multicolumn{2}{|l|}{ Plants } & \multirow{2}{*}{$\begin{array}{r}\text { Plants } \\
.1 \mathrm{oz} .1500\end{array}$} \\
\hline & $\ldots 1$ oz. 300 & Marjoran & \\
\hline Cabbage & $.1 \mathrm{oz} .2000$ & Pepper & $.1 \mathrm{oz} .1500$ \\
\hline Cauliflower & $\ldots 1$ oz. 1500 & Rhubarb & .1 oz. 500 \\
\hline Celer & $\ldots 1$ oz. 2500 & Tomato & .1 oz. 1500 \\
\hline Endive & $\ldots 1$ oz. 3000 & Thyme & .1 oz. 4000 \\
\hline Egg Plant & 1 oz. 1500 & Sage .... &. .1 oz. 1 \\
\hline Leek & 1 oz. 1000 & Savory & 2000 \\
\hline
\end{tabular}

TABLE SHOWING THE NUMBER OF PLANTS OR TREES TO THE ACRE AT GIVEN DISTANCE.

\begin{tabular}{|c|c|c|c|c|}
\hline Dist & apar & No. trees & Dist. apart & No. trees \\
\hline 1 & foot & $.43,500$ & 9 feet....... &. .537 \\
\hline $11 / 2$ & feet & $.19,360$ & 12 feet. & .302 \\
\hline 2 & feet & (10,890 & 15 feet... & .193 \\
\hline $21 / 2$ & feet & $\quad 6,960$ & 18 feet........ &. .134 \\
\hline 3 & feet & 4,480 & 21 feet. & 98 \\
\hline 4 & feet & $\ldots .2,722$ & 24 feet.. & 75 \\
\hline 5 & feet & 1,740 & 27 feet.. & .... 59 \\
\hline & feet & 1,210 & 38 feet....... & $\ldots 30$ \\
\hline
\end{tabular}




\section{WEIGHT OF DIFFERENT MANURES TO APPLY TO DIFFERENT CROPS PER ACRE.}

Potatoes

Stable 15 to 30 tons

Mangel wurzel Stable 20 to 30 tons

Carrots Stable 12 to 20 tons

Beans

Stable 12 to 20 tons

Onions

Stable 25 to 40 tons

Nitrate of soda and potash

250 lbs.

Salt 250 to $500 \mathrm{lbs}$.

Soot 1000 to $1500 \mathrm{lbs}$.

Concentrated fertilizers for garden or field cultures......500 to $800 \mathrm{lbs}$.

\section{BENEFITS OF MANURE.}

The benefit at any particular sample of manure is difficult to estimate, owing tc the fact that it may act upon the soil in several different ways, any of which, however, is invigorating; and the effect of manure extends over a period of years. Taking the subject as a whole, the benefits secured from applying the manure to the soil may be summed up as follows:

First-Manure increases the supply of humus.

Second-It adds plant food.

Third-It acts upon the soil in such a manner as to render plant food in the soil more available.

Fourth-It makes the soil warmer.

Fifth-It enables the soil to receive and retain more water and give it up gradually to growing crops.

Sixth-It improves soil ventilation.

Seventh-It aids in the development of bacteria.

Eighth-It helps to prevent the denuding effect of washing and heavy wind storms.

\section{VALUE OF OTHER FOODS COMPARED WITH HAY.}

It has been estimated that one hundred pounds of good hay will possess the same feeding value as

\begin{tabular}{|c|c|}
\hline $\begin{array}{r}\text { Pounds } \\
44 / 2\end{array}$ & $\begin{array}{l}\text { Pounds } \\
59\end{array}$ \\
\hline$-62 \frac{1}{2}$ & Rye \\
\hline lover, Red, Greent... & Potatoes \\
\hline eets 669 & Peas, dry. \\
\hline eans & .347 \\
\hline arley & Clover, Red Dry... \\
\hline 371 & 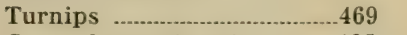 \\
\hline ........ 43 & Carrot leaves \\
\hline uckwheat & Rye-Straw ....... \\
\hline
\end{tabular}




\section{SPRAYING SUGGESTIONS.}

Don't spray immediately after a rain or heavy dew.

Spray on a still day. In you must spray in the wind, spray the windward side and leave the other till the wind changes or a still day comes.

Don't spray when trees are in full bloom. It reduces the crop and kills the bees.

Arsenical sprays should not be applied within two weeks of the time the fruit is to be eaten.

\section{MEASURING AND ESTIMATING HELPS.}

\section{Rule for Measuring Corn.}

A heaped bushel contains 2748 cubic inches. To find the number of bushels of corn in a crib it is therefore necessary merely to multiply together the length, width and height in inches and divide the product by 2748 . The number of bushels of shelled corn will be two-thircis of the quotient. If the sides of the crib are slanting, it will be necessary to multiply together one-half the sum of the top and bottom widths, the height and length.

\section{Rule for Estimating Hay.}

Hay is often sold in the mow or stack where the weight has to be estimated. For this purpose 400 cubic feet of hay is considered a ton. The actual weight of 400 cubic feet of hay will vary according to the quality of the hay, time of cutting, position in the mow, etc. For making an estimate in a given case multiply together the length, breadth and height of the mow or stack in feet and divide the product by 400 . The quotient will be the number of tons.

\section{Grain Estimates.}

A wagon box ten feet long, three feet wide and 25 inches deep will hold 27.8 bushels of ear corn, or 50.2 bushels of shelled corn. A crib 10 feet wide, 10 feet high and 16 feet long will hold 711 bushels of ear corn. Of ear corn one bushel is contained in two and a quarter cubic feet. In figuring shelled corn and grain, the same space will hold one and four-fifths times as much grain as it will of ear corn. A crib that will hold 800 bushels of ear corn will hold of shelled corn or other grain 1,440 bushels.

\section{Weights for Barn Use.}

Frequently the farmer wishes to feed a given weight of this, that or the other stuff and has no scales at hand to weigh it. If he has a quart measure handy, he can use it to measure out the required weight. The quart weight of various feeds is as follows:

Cottonseed meal, 1.5 pounds; linseed meal, old process, 1.1 pounds; gluten meal, 1.7 pounds; gluten feed, 1.2 pounds; wheat bran, coarse, .5 pound; wheat middlings, coarse, .8 pound, and fine, 1.1 pounds; mixed wheat feed, .6 pound; cornmeal, 1.5 pounds; oats, 1.2 pounds; rye bran, .6 pound; H. O. dairy feed, .7 pound, and Victor corn feed, .7 pound per quart. 


\section{THE COST OF RAISING A DAIRY COW.}

(U. S. DEPT. AGR. BULLETIN 49)

One-Year Old Per cent. Two-year Old Per cent.

\begin{tabular}{|c|c|c|c|}
\hline Heifer & & Heifer & \\
\hline Initial value & 17 & $\$ 7.04$ & 11.4 \\
\hline Feed & 62 & 40.83 & 66.4 \\
\hline Labor & 11 & 7.81 & 12.7 \\
\hline Other items $\quad 6.36$ & 16 & 13.73 & 22.3 \\
\hline Total & & $\$ 69.41$ & \\
\hline Credit manure ............... 3.00 & 7 & 8.00 & 13 \\
\hline Net cost & & $\overline{\$ 61.41}$ & \\
\hline
\end{tabular}

\section{AVERAGE COST OF RAISING A HORSE.}

(FROM INIESTIGATIONS OF THE U. S. DEPT. OF AGRICULTURE)

Service fee

Time lost by mare in foaling.

10.06

Breaking to halter.

2.22

Veterinary service

Care and shelter - 1st year, $\$ 4.98 ; 2$ d year, $\$ 5.36 ; 3$ d year, $\$ 6.35 . .16 .69$

Grain fed

Hay fed

Pasture

Other costs

Total 1st year, $4.98 ; 2 \mathrm{~d}$ year, $7.14 ; 3 \mathrm{~d}$ year, 9.56 .21 .68 1 st year, $4.14 ; 2$ d year, $6.61 ; 3 \mathrm{~d}$ year, $8.48 . .18 .23$ 1 st year, 2.56; 2d year, $5.41 ; 3 \mathrm{~d}$ year, 6.21 .. 14.18 Island.

Range included from $\$ 69.50$ in New Mexico to $\$ 156.60$ in Rhode

\section{HOW TO MAKE WHITEWASH.}

(From Farmer's Bulletin No. 474)

For interior work: Walls, ceilings, etc.:

(1) Slake 62 pounds (1 bushel) quicklime with 15 gallons water. Keep barrel covered until steam ceases to rise. Stir occasionally to prevent scorching.

(2) Two and one-half pounds rye flour. Beat up in $1 / 2$ gallon cold water, then add two gallons boiling water.

(3) Two and one-half pounds common rock salt. Dissolve in $2 \frac{1}{2}$ gallons hot water.

Mix (2) and (3), pour into (1) and stir till thoroughly mixed.

For exterior work: Buildings, fences, etc.:

(1) Sixty-two pounds (1 bushel) quicklime. Slake with 12 gallons hot water.

(2) Two pounds common table salt, one pound sulphate of zinc, dissolved in two gallons boiling water.

(3) Two gallons skimmed milk.

Pour (2) into (1), then add (3) and mix thoroughly.

A pound of cheap bar soap dissolved in a gallon of boiling water and added to about five gallons of thick whitewash will give it a gloss like that of oil point. 


\section{BRIEF BUSINESS LAWS.}

The maker of a note that is lost or stolen is not released from payment if the amount and consideration can be proven.

Notes bear interest only when so stated.

Demand notes are payable on presentation, without grace, and bear legal interest after a demand has been made, if not so written. An endorser on a demand note is holden only for a limited time, variable in different states.

A negotiable note must be made payable either to bearer, or be properly endorsed by the person to whose order it is made. If the endorser wishes to avoid responsibility, he can endorse "without recourse."

A joint note is one signed by two or more persons, who can each become liable for the whole amount.

No grace is allowed in the majority of states on time notes after the time for payment expires. If not paid when due, the endorser, if any, should be legally notified to be holden.

Notes falling due on Sunday, or on a legal holiday, must be paid the day following as a rule.

A note made on Sunday is void, also one dated ahead of its issue. It may be dated back at pleasure.

Contracts made on Sunday cannot be enforced.

Altering a note in any manner, by the holder, makes it void.

A note by a minor is void in some states, and in others is voidable on judicial decision.

A contract made with a minor, or a lunatic, is void.

Notes obtained by fraud, or given by intoxicated persons, cannot be collected. It is a fraud to conceal a fraud. Signatures made with a lead pencil are good in law. The acts of one partner bind the rest.

Principals are responsible for the acts of their agents.

Each individual in a partnership is responsible for the whole amount of the debts of the firm, except in cases of special partnership. The word "limited," in connection with a firm name, indicates that a limitation of responsibility for each member is fixed.

Ignorance of the law excuses no one. A receipt for money is not legally conclusive.

An agreement without consideration of value is void. "Value received" is usually written in a note, and should be, but it is not necessary. If not written, it is presumed by the law or may be supplied by proof. 


\section{General Index}

\section{Horse Section}

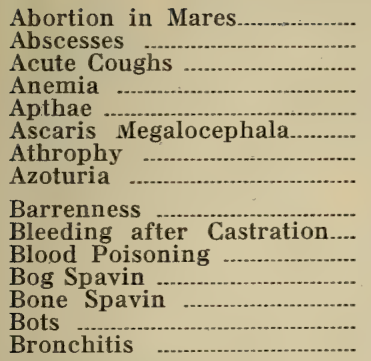

Capped Elbow .................-....... 48

Capped Hock .......................... 16

Capped Knee .............................. 16

Cerebral Meningitis ................ 45

Choking ……......................... 16

Chronic Coughs …--............... 21

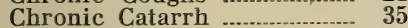

Coffin Joint Lameness............. 16

Colds ......................................... 20

Colic, Spasmodic ……-... 19

Colic, Wind or Flatulent........ 20

Colt Constipation …-.....-......-... 18

Colt Diarrhoea ........................ 18

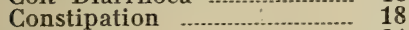

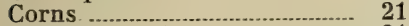

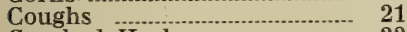

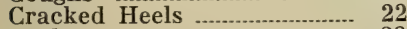

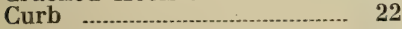

Diarrhoea ……-.................... 23

Diseases of the Eye -..-.-.... 25

Dislocation of the Patella....

Distemper .............................

Dropsy of the Belly

Dropsy of the Chest................

Dropsy of the Legs ...............

Dropsy of Udder or Sheath

Eczema

Emphysema of the Lungs..-

Epizootic Catarrh

Eye Diseases

Failure to Breed

Farcy

Filariae

Fistula

Fistula of the Food

Fistulous Withers .

Forage Poisoning

Founder
Galls …......................................... 27

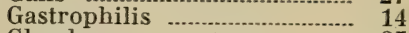

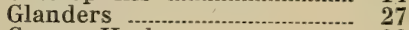

Grease Heel ……..................... 28

Haemopis ............................... 31

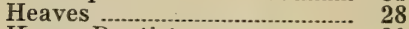

Horse Dentistry ….............- 30

How to Find Your Horses' Age .................................... 29

Inflammation of the Brain.... 45

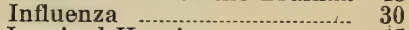

Inguinal Hernia ........-..-......... 45

Larngitis ................................ 46

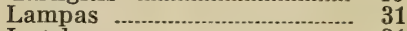

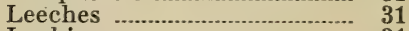

Lockjaw ….................................. 31

Lung Fever

Lymphangitis …….................. $\quad 34$

Mange ……............................. 34

Monday Morning Disease...... 34

Mud Fever ......................... 35

Nasal Catarrh …................... 20

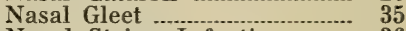

Navel String Infection........... 36

Navel String Rupture............. 38

Navicular Disease .......................... 16

Nettle Rash ............................ 51

Open Joint .............................. $\quad 39$

Oxyuris Curvula .................... 41

Palisade Worm ..._............... 39

Patechial Fever ........................ 40

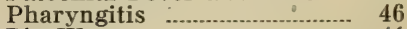

Pin Worm ............................. 41

Pink Eye ..................................... 30

Pleurisy …………........................... 41

Pneumonia ...................-...-....... 32

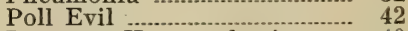

Purpura Haemorrhagica ...... 40

Pyaemia ….............................. 14

Quittor ............................ 43

Red Worm ……_..._................... 43

Rheumatism …........................... 44

Ring Bone ............................................ 43

Round Worm ........................ 44

Scabies ……......................... 34

Scrotal Rupture …................-. 45

Septicaemea _.............................. 14

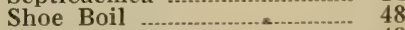

Side Bones ……......................... 48

Sore Throat ……................................ 46

Spavin, Bog ........................... 48 
Spavin, Bone

49 Thoroughpin

Splints

Staggers

Strangles

Stifle Joint Lameness.

String Halt

Strongylus Armatus

Thread-like

Armatus ...............

Strongylus Tetracanthus

Surfeit

Thread or Maw Worm

Thrush

Umbilical Pyaemia

Umbilical Hernia

Urtecaria

Taenia

Tape Worm

Wind Colic

Wind Galls

Tetanus

Wolf Teeth

Wounds

\section{Cattle Section}

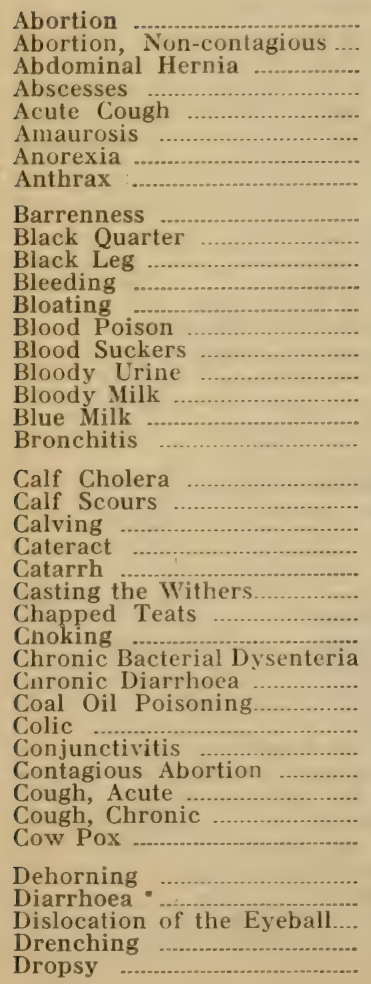

59

59

88

60

$6 \dot{0}$

82

59

64

64

64

63

65

62

83

89

63

63

62

69

69

66

67

67

70

71

70

71
Eczema

Ergot Poisoning

Ergotism

Eversion of Womb.............. 70

Feeds and Feeding .................. $\quad \mathbf{7 5}$

Fluke in Liver and Lungs.... 75

Founder .............................. 75

Garget …................................. 72

Grubs ......................................... 91

Haemorrhage ......................... 63

Hard Milkers ................................ 77

Hollow Horn .......................... 76

How to Administer Dr. Korinek's Capsules .................... 77

How to Care for the Calf..... 76

Indigestion ............................... 83

Infectious Abortion ............... 81

Inflammation of the Eye...... 77

Inflammation of the Kidneys 78

Inflammation of the Penis.... 78

Inflammation of the Womb.. 78

Inflammation of the Heart Sac Inflammation of the Bag..... 79

Laceration of the Eyeball.... 84

Laminitis ……........................ 75

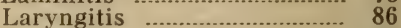

Leeches ........................................ 83

Leucorrhoea ……................... 91

Loss of Appetite........................ 82

Loss of Cud............................. 83

Lung Fever ............................... 84

Lung Worm .................................. 94

Mammitis _............................... 79

Measley Beef ........................... 84

Metritis ................................. 78

Milk Fever ................................. 85

Navel IIl ............................... 85 
Paralysis

Parturient Apoplexy

Pericarditis

Physiology of Rumination....

Points of the Cow.

Pyaemia

Red Water

Retained Afterbirth

Rheumatism

Ring Worm

Ringing the Bull

Round Worm

Rupture

Symptomatic Anthrax

Stringy Milk
86

85

79

86

58

62

89

89

89

90

86

88

88 .

64

90
Scum on the Eye.

Southern Cattle Fever.

Suppression of Milk.

Tape Worms

Texas Fever

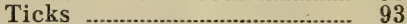
Tuberculosis …………......... 92

Twisted Stomach Worms...... 93 Verminous Bronchitis .......... 94

Warbles

Warts on Teats.

White Scours

Whites

Wolf in Tail
91 93 91

94 93 91 95 69 91 95

\section{Swine Section}

Abortion

Administration of Medicire to Hogs

99

99

Bag Inflammation 102

Black Tooth 100

Blood Poisoning

Bronchitis

99

Castration 100

Choking

101

Cholera 101

102

Diarrhoea in Young Pigs...... 102

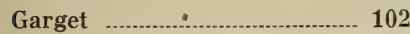

Heat Stroke

114

Hog Cholera 102

Indigestion 104

Inflammation of the Bag....... 102

Inflammation of the Lungs.. 106

Inguinal Rupture 113

Jaundice 104

Kidney Congestion 105

Kidney Worm 104

Lice 106

Lung Fever 106

Lung Worms 105

Mange 106

Navel Rupture

Nettle Rash
Paralysis of the Hind Quarters 110

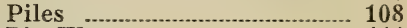

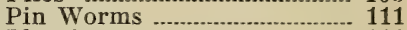

Pleurisy ………………............... 111

Points of the Hog.................. 98

Prolapsus of the Anus.............. 108

Pyaemia ………........................ 99

Rheumatism ….......................... 112

Rickets …................................................ 114

Round Worms .......................... 112

Rupture, Inguinal ...................... 113

Rupture, Scrotal ...................... 113

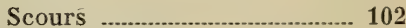

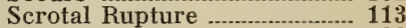

Septicaemia …-...-...-...-...-....... 99

Serum Treatment for Hog Cholera .......................... 115

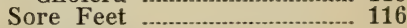

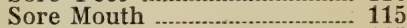

Sows Eating Pigs............-......... 115

Sun Stroke ........................... 114

Thorn Headed Worms........... 116

Thumps ……........................... 117

Umbilical Rupture .................... 108

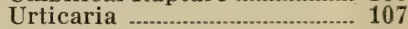

Whip Worm …_-................... 117

Worms, Round …--...-............. 112

Worms, Kidney …..................... 104

Worms, Lung …-...-..................... 105

Worms, Pin ……...................... 111

Worms, Thorn-headed ........... 116

Worms, Whip ………….......... 117

107. Yellows ….................................. 104 


\section{Sheep Section}

Bad Cold
Black Scours
Bloating
Bronchitis Verminous

\section{Poultry}

Abortion

Abscesses

Air Sac Mite.

Apoplexy

Ascites

Baldness

Beak and Throat Obstruction

Black Head

Body Lice

Bronchitis

Bruises

Bumble Foot

Catarrh

Chicken Pox

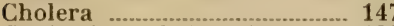

Conjestion of the Liver........ 138

Conjestion of the Lungs......... 138

Constipation …....................... 139

Corns ……_........................... 135

Crop Impaction _.._............ 139

Deep Bruises …_-_................... 135

Difficult Laying _._._._._-_...-...- 141

Diptheretic Roup :-................... 140

Diptheria …………....................... 140

Double Yolk Eggs ……-............. 141

Dropsy .............................. 141

Egg Bound ............................ 141

Egg Eating ……………….... 142

Eggs without Shells ……..... 142

Enteritis

Favus

Feather Pulling

134

Feather Eating

Fowl Cholera

Gapes

Gastro-Intestinal Catarrh.

Gastro-Enteritis

Gout
Leech or Liver Fluke

Lice and Leg Disease

Lip and Leg Ulceration

Louse Fly

Mange 126

Points of the Sheep 120

Sheep Ticks

Sheep Pox

Sheep Variola

Strongylosis 128 128 128

Verminous Bronchitis 128

\section{Section}

Head Lice .............................. 143

Hemmorrhage of the Brain.. 133

How to Feed Young Chicks 143

Incomplete Egg .................... 144

Infectious Entero-Hepatis of Turkeys ....................... 134

Inflammation of Crop ......... 139

Inflammation of the Mouth 144

Intestinal Obstruction ............ 139

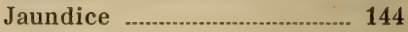

Leg Weakness ……................... 145

Liver Congestion ...................... 144

Mange _.................................. 144

Obstruction of the Oviduct.. 141

Paryalsis ........................... 145

Paralysis of the Crop........... 139

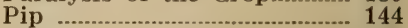

Points of the Fowl ............. 132

Red Mites .................................. 145

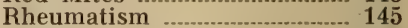

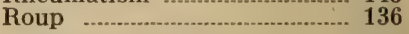

Scabies of the Body ............ 144

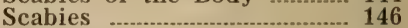

Scaly Leg ………................................. 146

Soft Shelled Eggs _-_ _-_...-. 142

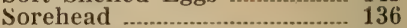

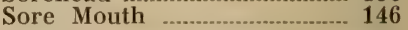

Throat Obstruction …............ 133

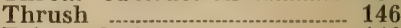

Tuberculosis ………................ 146

Vent Cleet ……_......... 147

Verminous - Tracheo Bronchitis …................................ 142

Warts …_................................. 146

White Diarrohea …………….... 147

Worms …..................-........... 148 
Dog Section

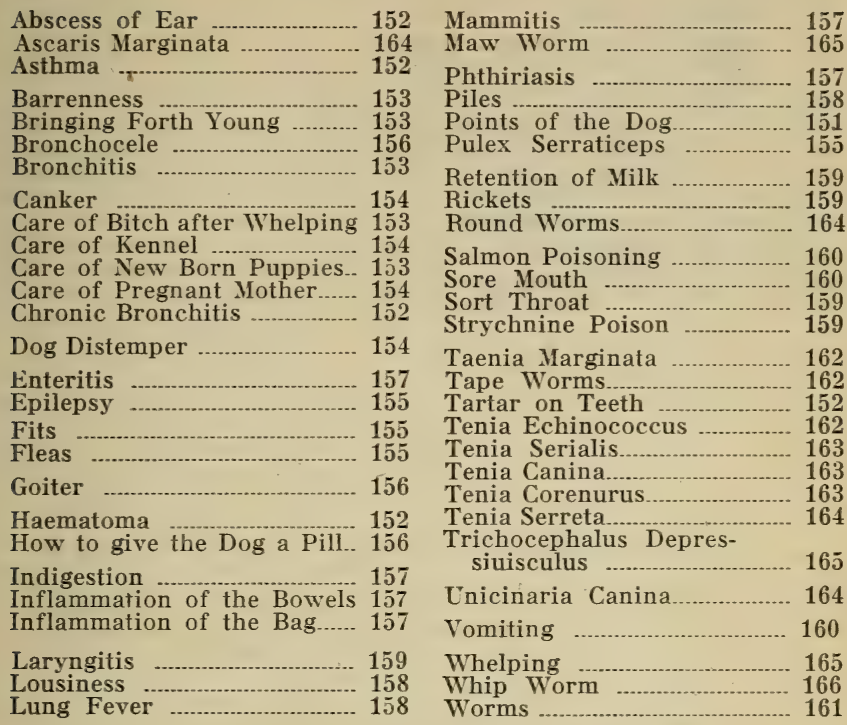

\section{Useful Information for the Farmer}

Age of Puberty

Antidotes for Poisons

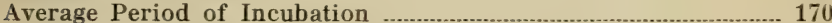

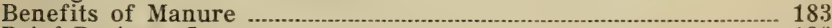

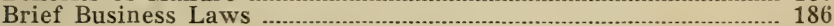

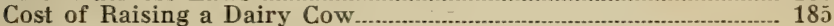

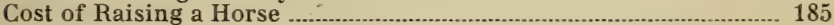

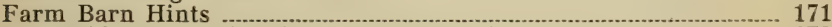

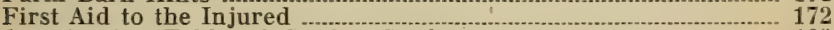

Germinating Table of Garden Seeds............................................. 182

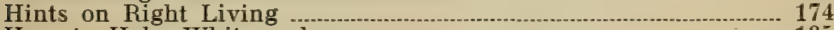

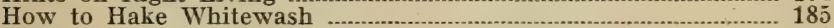

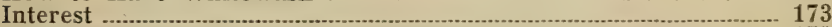

Legal Weights of Various Commodities …

Length of Germ Life in Seeds................................................... 181

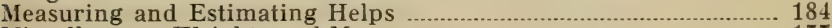

Miscellaneous Weights and Measures …_........................................ 177

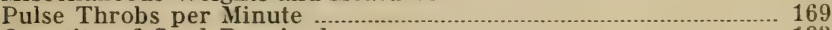

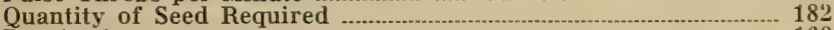

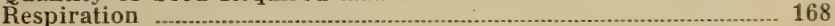

Some Facts about Stock Food _._-_._- 170

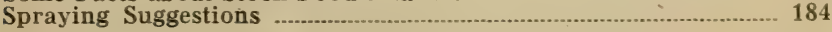


Special Poisons and Antidotes 175

Tabloid Recipes

Temperature of Cattle

Temperature of the Dog

Temperature of Sheep and Goats

168

Temperature of the Horse

Temperature of Swine

168

(T)

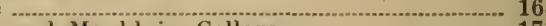

.

Value of Other Foods Compared with Hay

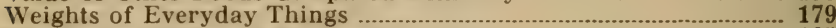

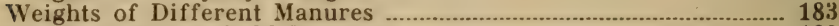

Weights of Household Measures ……......................................... 180

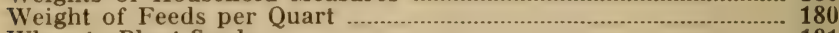

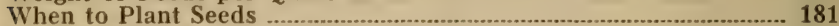

What To Do 'Till the Detober Comes........................................... 173 

LIBRARY OF CONGRESS

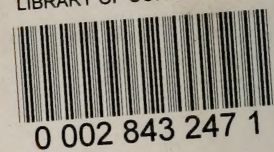

